\title{
A Wearable Computer Vision System for Measuring Shoe Position and Orientation
}

by

\author{
Ayron R. Catteau \\ A Thesis submitted to \\ the Faculty of Graduate Studies and Research \\ in partial fulfilment of \\ the requirements for the degree of \\ Master of Applied Science \\ Department of Mechanical \& Aerospace Engineering \\ Carleton University \\ Ottawa, Ontario, Canada
}

August 2014

Copyright (c)

2014 - Ayron R. Catteau 


\begin{abstract}
The BalanceAid project was started by the Advanced Biomechatronics and Locomotion Laboratory at Carleton University to create a wearable system capable of aiding those with balance related disabilities. This thesis presents, as a component of the BalanceAid project, the initial design and prototype of a self-contained wearable system capable of measuring the relative position and orientation of the wearer's shoes. This system is the first published work to use two shoe mounted cameras to measure the position and orientation of the shoes during the entire gait cycle in real time and using entirely on-board processing.

The system consists of a camera, eight LED markers, and a single board computer mounted to each shoe. All of the data processing is performed on the shoe mounted computers providing a 6 DoF coordinate system transformation from one shoe to the other at a rate of $15 \mathrm{~Hz}$.

Using 6 healthy subjects, experiments were performed to characterize the performance of the system. The system's measurements were compared to those of an accurate commercially available system. The shoe localization system was able to correctly calculate the position and orientation between the shoes with $90 \%$ of the measurement errors being less than $10.85 \mathrm{~cm}$ and $25.19 \mathrm{deg}$. Overall, the experiments demonstrate that the shoe localization system is successful, but further development is required to eliminate anomalous measurements and reduce the susceptibility of the system to sources of error.
\end{abstract}


To my wife. 


\section{Acknowledgments}

This thesis would not be what it is today without the help of many people. I would like to express my sincere gratitude to my supervisor Prof. Mojtaba Ahmadi for all of his continual guidance and support.

I would like to thank my research partner Patricia Giacoman Zarzar, with whom I have shared this project. I could not have asked for a better colleague. I would also like to thank our summer student Stephanie Eng, who took on the project of the vibrotactile belt when we had too much on our plates.

Finally, I would like to thank the lab technologists who helped me with the prototype construction: Kevin Sangster, Alex Proctor, and Stephan Biljan. 


\section{Contents}

Abstract ii

List of Figures viii

List of Tables $\quad x$

List of Algorithms $\quad$ xi

Nomenclature xii

$\begin{array}{ll}\text { 1. Introduction } & 1\end{array}$

1.1. Motivation . . . . . . . . . . . . . . . . 1

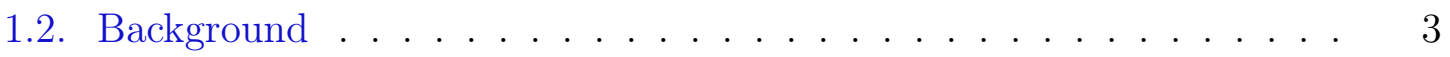

1.2.1. Balance Aid . . . . . . . . . . . . . . . . . 3

1.2.2. Gait Analysis . . . . . . . . . . . . . . . 4

1.2.2.1. Center of Pressure . . . . . . . . . . . . 5

1.2.3. Pedestrian Localization . . . . . . . . . . . . . . . . . 6

1.2.4. Coordinate System and Transformation Notation . . . . . . 7

1.2.5. Localization Technology . . . . . . . . . . . . . . . . . . 11

1.2.6. Optotrak Certus . . . . . . . . . . . . . . . . 13

1.2.7. Related Work . . . . . . . . . . . . . . . . . . . . 15

1.2.7.1. Balance Aid . . . . . . . . . . . . . . . 15

1.2.7.2. Gait Analysis . . . . . . . . . . . . . . 17

1.2.7.3. Pedestrian Localization . . . . . . . . . . . 18

1.3. BalanceAid Project Overview . . . . . . . . . . . . . . . . . . . . . 19

1.4. Localization System Overview . . . . . . . . . . . . . . . 20

1.4.1. Principle of Operation . . . . . . . . . . . . . . . 22

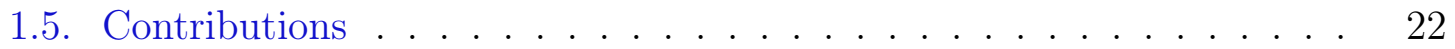

1.6. Thesis Overview . . . . . . . . . . . . . . . . . . . . . 23

2. Hardware Design 25

2.1. Overview . . . . . . . . . . . . . . . . . . . . . . 25

2.2. Design Considerations . . . . . . . . . . . . . . . . 26

2.2.1. Design Limitations . . . . . . . . . . . . . . . . . . . 28

2.3. Raspberry $\mathrm{Pi} \ldots \ldots \ldots \ldots$

2.4. Camera Board, Lens, and Filter . . . . . . . . . . . . . . . 31

2.5. IR Markers . . . . . . . . . . . . . . . . . . . . . 34

2.6. Shoe and Structure . . . . . . . . . . . . 36 
2.7. Chapter Summary . . . . . . . . . . . . . . . . . . . . . . . . . . . 38

3. Software Design 40

3.1. Overview . . . . . . . . . . . . . . . . . . . . . 40

3.2. The Shoevision Process . . . . . . . . . . . . . . . . . . . . 42

3.2.1. Connected Component Labelling _. . . . . . . . . . . . 43

3.2.2. Blob Filtering . . . . . . . . . . . . . . . . . . . . 48

3.2.3. The Find_Solution Algorithm . . . . . . . . . . . 50

3.2.4. The Camera Model . . . . . . . . . . . . . . . . . . . . 55

3.2.5. The Find_Front/Rear_Set_Solution Algorithms ... 62

3.3. Integrator $\ldots \ldots \ldots \ldots \ldots \ldots \ldots \ldots \ldots \ldots \ldots$

3.4. Chapter Summary . . . . . . . . . . . . . . . . . . . . . 73

3.4.1. Recommendations . . . . . . . . . . . . . . . . . 73

4. Calibration 75

4.1. Overview . . . . . . . . . . . . . . . . . 75

4.2. Calibration Board Transformation . . . . . . . . . . . . 77

4.2.1. Measurement . . . . . . . . . . . . . . . . . . . 77

4.2.2. Data Processing . . . . . . . . . . . . . . . . . . . 78

4.2.3. Results . . . . . . . . . . . . . . . . . . . . 80

4.3. Camera Calibration . . . . . . . . . . . . . . . . . 80

4.3.1. Measurement . . . . . . . . . . . . . . . . . 81

4.3.2. Data Processing . . . . . . . . . . . . . . . . . . . . . 82

4.3.3. Results . . . . . . . . . . . . . . . . . . . . 84

4.4. Rear Shoe Optotrak Marker Transformation . . . . . . . . . . . . 84

4.4.1. Measurements . . . . . . . . . . . . . . . . . . . . 85

4.4.2. Data Processing . . . . . . . . . . . . . . . . . . . . . . 85

4.4.3. Results . . . . . . . . . . . . . . . . . 86

4.5. IR LED Marker Locations . . . . . . . . . . . . . . . 86

4.5.1. Measurement . . . . . . . . . . . . . . . . . . 87

4.5.2. Data Processing . . . . . . . . . . . . . . . . . . . . 88

4.5.3. Results . . . . . . . . . . . . . . . . . . . . . 89

4.6. Chapter Summary . . . . . . . . . . . . . . . . . . . . . . . 89

5. Tests \& Characterization $\quad 91$

5.1. Experiment Overview . . . . . . . . . . . . . . . . . . . . . 91

5.2. Short Walk . . . . . . . . . . . . . . . . . . . . . . . . . 92

5.2.1. Measurement . . . . . . . . . . . . . . . . . . . . . . . . 93 93

5.2.2. Data Processing . . . . . . . . . . . . . . . . . . . 93

5.2.3. Results . . . . . . . . . . . . . . . . . . . . . . . 95

5.2.4. Discussion . . . . . . . . . . . . . . . . . . . . . 96

5.3. Long Walk . . . . . . . . . . . . . . . . . . . . . . . . 100

5.3.1. Measurement . . . . . . . . . . . . . . . . . . . . . 100

5.3.2. Data Processing . . . . . . . . . . . . . . . . . . . . . . . . 101

5.3.3. Results . . . . . . . . . . . . . . . . . . . . . . . 104 
5.3.4. Discussion . . . . . . . . . . . . . . . . 105

5.4. Chapter Summary . . . . . . . . . . . . . . . . . . 107

$\begin{array}{ll}\text { 6. Conclusion } & 108\end{array}$

6.1. Recommendations for Future Work . . . . . . . . . . . . . . . . 109

$\begin{array}{ll}\text { References } & 111\end{array}$

$\begin{array}{ll}\text { A. Software } & 116\end{array}$

$\begin{array}{ll}\text { B. Calibration Results } & 117\end{array}$

$\begin{array}{ll}\text { C. Additional Test Results } & 120\end{array}$ 


\section{List of Figures}

1.1. Diagram of ground reaction forces and CoP underneath a pair of shoes. 5

1.2. Two coordinate systems $A$ and $B$, and a point $C$, shown with connecting vectors. . . . . . . . . . . . . . . 8

1.3. The hardware components of the Optotrak Certus measurement system. 14

1.4. An Optotrak marker triplet located on the rear of each shoe. . . . . 15

1.5. Block diagram description of feedback of center of pressure information. 20

1.6. Overview of the localization system design. . . . . . . . . . . . 21

2.1. Hardware assembly used in localization system. . . . . . . . . . . 26

2.2. Raspberry Pi Model B single board computer with labels. . . . . . . 29

2.3. The camera assembly supported on each shoe. . . . . . . . . . . . 31

2.4. Field of vision of each camera during different stages of walking. . . 33

2.5. Infrared LED used as a marker in the localization system. . . . . . . 34

2.6. Visibility of the two marker sets. . . . . . . . . . . . . . 35

2.7. Skeletal support structure shown with and without shoe deformation. 37

3.1. Overview of software architecture. . . . . . . . . . . . . 41

3.2. Geometric depiction of the localization solution. . . . . . . . . . . . 42

3.3. Overview of the shoevision process. . . . . . . . . . . . . . 43

3.4. Example of a captured image with labels. . . . . . . . . . . . 45

3.5. Visualization of the output of connected component labelling. . . . 48

3.6. Visualization of output of blob filtering. . . . . . . . . . . . . 49

3.7. Geometry of the camera model with labels. . . . . . . . . . . . . 55

3.8. Depiction of parameters used in the camera model. . . . . . . . . 59

3.9. Marker arrangement in the two marker sets. . . . . . . . . . . 63

3.10. Depiction of values used in Fabrizio and Devars' solution to the P4P problem using coplanar points. . . . . . . . . . . . . 65

3.11. Geometry of a simple attitude estimation problem. . . . . . . . . . 67

3.12. Geometry of coordinate systems and markers for the shoe localization problem. . . . . . . . . . . . . . . . 68

3.13. Overview of the integrator process. . . . . . . . . . . . . . 71

4.1. Coordinate systems used in the calibration process. . . . . . . . 76

4.2. The location of the three points measured by the Optotrak probe. . 78

4.3. The location of the corners in the calibration pattern. . . . . . . 81

4.4. Measurement of the IR LED locations. . . . . . . . . . . . . . 87

4.5. The IR LED markers that must be measured during calibration. . . 87 
5.1. Layout of the short walk experiment. . . . . . . . . . . . . . . . . . 92

5.2. Photo of the short walk experiment. . . . . . . . . . . . 93

5.3. Frequency of translation and rotation errors. . . . . . . . . . . 96

5.4. Plots of translation and rotation error for a single subject during a single short walk. . . . . . . . . . . . . . . . . . . 97

5.5. The average translation and rotation errors during a single step. . . 98

5.6. Distance between the camera frames. . . . . . . . . . . . . . . 99

5.7. Path followed by subjects during the long walk experiment. . . . . . 101

5.8. The path of a subject estimated from the tracking algorithm. . . . . 104

5.9. The path of each circuit made by a subject estimated from the tracking algorithm. . . . . . . . . . . . . . . . . . . 105

5.10. The results of the tracking algorithm compared to the true steps as measured by the Optotrak. . . . . . . . . . . 106

C.1. The path of each circuit made by subject 1 estimated from the tracking algorithm. . . . . . . . . . . . . . . . . . 120

C.2. The path of each circuit made by subject 2 estimated from the tracking algorithm. . . . . . . . . . . . . . . . . . . 121

C.3. The path of each circuit made by subject 3 estimated from the tracking algorithm. . . . . . . . . . . . . . . . . . . . 122

C.4. The path of each circuit made by subject 4 estimated from the tracking algorithm. . . . . . . . . . . . . . . . . . . . . 123

C.5. The path of each circuit made by subject 5 estimated from the tracking algorithm. ................... 


\section{List of Tables}

2.1. Selected technical specifications of the Raspberry Pi Model B. . . . 30

2.2. Selected technical parameters of the Pi NoIR camera module. . . . . 32

3.1. Tunable parameters used in the camera model. . . . . . . . . . 58

B.1. Location of the eight markers in the camera frame of each shoe. . . 117

B.2. Parameters used in Fabrizio's solution for the front and rear marker set of each shoe. . . . . . . . . . . . . . . . . . . . . 118

B.3. Coordinate system transformation from the camera frame to the rear frame for each shoe. . . . . . . . . . . . . . . . . . 118

B.4. Camera model parameters for both shoes. . . . . . . . . . . . . 119 


\section{List of Algorithms}

3.1. Pseudocode for connect component labelling algorithm. . . . . . . . . 47

3.2. Pseudocode for the FIND_SOLUTION algorithm. . . . . . . . . . . . 51

3.2. Pseudocode for the FIND_SOLUTION algorithm. (continued) . . . . . 51

3.2. Pseudocode for the FIND_SOLUTION algorithm. (continued) . . . . . 52

3.2. Pseudocode for the FIND_SOLUTION algorithm. (continued) . . . . . 53

3.2. Pseudocode for the FIND_SOLUTION algorithm. (continued) . . . . 53

3.2. Pseudocode for the FIND_SOLUTION algorithm. (continued) . . . . . 54

3.2. Complete pseudocode for the FIND_SOLUTION algorithm. . . . . . . 54 


\section{Nomenclature}

$\begin{array}{ll}\text { Abbreviations } \\ \text { ABL } & \text { Advanced Biomechatronics and Locomotion } \\ \text { CCL } & \text { Connected Component Labelling } \\ \text { CoP } & \text { Center of Pressure } \\ \text { CPU } & \text { Central Processing Unit } \\ \text { CSI } & \text { Camera Serial Interface } \\ \text { DFS } & \text { Depth First Graph Search } \\ \text { DoF } & \text { Degree of Freedom } \\ \text { EM } & \text { Electromagnetic } \\ \text { FoV } & \text { Field of View } \\ \text { GNSS } & \text { Global Navigation Satellite System } \\ \text { GPS } & \text { Global Positioning System } \\ \text { GPU } & \text { Graphics Processing Unit } \\ \text { HDMI } & \text { High-Definition Multimedia Interface } \\ \text { IMU } & \text { Inertial Measurement Unit } \\ \text { INS } & \text { Inertial Navigation System } \\ \text { IPS } & \text { Indoor Positioning System } \\ \text { IR } & \text { Infrared } \\ \text { LED } & \text { Light Emitting Diode } \\ \text { PnP } & \text { Perspective N Point } \\ \text { Tactors } & \text { Vibrotactile Actuators } \\ \text { UKF } & \text { Unscented Kalman Filter } \\ \end{array}$




\section{USB Universal Serial Bus}

\section{General Notation}

$\Lambda_{A B} \quad$ The coordinate system transformation from frame $B$ to frame $A$

$\mathbf{q}_{A B} \quad$ The unit quaternion transformation from frame $B$ to frame $A$

$\hat{\mathbf{v}}_{A B} \quad$ The unit vector from $A$ to $B$

$\overrightarrow{\mathbf{v}}_{A B} \quad$ The vector from $A$ to $B$

$\mathbf{v}_{A B, A} \quad$ The $3 \times 1$ matrix of coordinates of $\overrightarrow{\mathbf{v}}_{A B}$ in frame $A$

\section{Coordinate Systems}

$B \quad$ Board frame. Attached to the Optotrak marker triplet on the calibration board.

C Camera frame. Could be on either shoe depending on context.

E Alias for the camera frame of the rear shoe in a walking stance.

F Alias for the camera frame of the forward shoe in a walking stance.

$H_{L} \quad$ Horizontal frame of the left shoe.

$H_{R} \quad$ Horizontal frame of the right shoe.

I Image frame. Coordinate system of the captured images, measured in pixels, centered at the top left corner.

$L \quad$ The camera frame on the left shoe.

O Rear frame. Attached to the Optotrak marker triplet on the rear of either shoe.

$O_{L} \quad$ Frame attached to the Optotrak marker triplet on the rear of the left shoe.

$O_{R} \quad$ Frame attached to the Optotrak marker triplet on the rear of the right shoe.

P Pattern frame. Attached to the calibration pattern.

$R \quad$ The camera frame on the right shoe.

$S \quad$ Sensor frame. Frame used in the camera model definition.

T Frame attached to the Optotrak tower. 


\section{Camera Model (Section 3.2.4)}

D Distorted Image Point

$\Delta_{r} \quad$ Radial distortion term

$D_{1}, \ldots, D_{5} \quad$ Coefficients of $\theta$ polynomial

$\Delta_{t} \quad$ Tangential distortion term

f $\quad$ Focal length

$I_{1}, \ldots, I_{4} \quad$ Fourier series coefficients of radial distortion

$J_{1}, \ldots, J_{4} \quad$ Fourier series coefficients of tangential distortion

$L_{1}, L_{2}, L_{3} \quad$ Polynomial coefficients of radial distortion

$M_{1}, M_{2}, M_{3}$ Polynomial coefficients of tangential distortion

$P \quad$ Undistorted Image Point

$r$, phi Polar coordinates of point on camera sensor

$S_{1}, S_{2} \quad$ The conversion factors from pixels to millimeters

$\theta, \phi \quad$ Spherical coordinates of direction of point in front of camera

$\hat{\mathbf{u}}_{D r} \quad$ Radial direction from distorted image point.

$\hat{\mathbf{u}}_{D t} \quad$ Tangential direction from distorted image point.

$p_{0}, q_{0} \quad$ Pixel coordinates of the image center in the image frame

$u, v \quad$ Coordinate of a pixel in the image frame.

Fabrizio's P4P Solution (Section 3.2.5)

$\Delta_{i j k} \quad$ determinant of the $3 \times 3$ matrix formed by horizontally stacking the column vectors $\mathbf{v}_{i}, \mathbf{v}_{j}$, and $\mathbf{v}_{k}$

$K_{1}, K_{2} \quad$ The coordinates of $\overrightarrow{\mathbf{v}}_{13}$ using $\overrightarrow{\mathbf{v}}_{12}$ and $\overrightarrow{\mathbf{v}}_{14}$ as a vector basis

$L_{12} \quad$ Distance from marker 1 to marker 2

$\lambda_{i} \quad$ Distance to marker i from the camera frame 


\section{Davenport's Q-Method (Section 3.2.5)}

$\mathbf{I}_{3} \quad 3 \times 3$ identity matrix

K Davenport matrix

\section{Calibration (Chapter 4)}

$d_{l} \quad$ The calibration pattern square side length in the length direction

$d_{w} \quad$ The calibration pattern square side length in the width direction

$f_{C I} \quad$ The camera model function: maps from image frame to camera frame

$\mathbf{P} \quad$ The 23 tunable parameters used in the camera model

\section{Tests \& Characterization (Chapter 5)}

$\hat{\mathbf{a}}$

A

$d_{e}$

$F_{L}$

$F_{R}$

$\Lambda_{\text {error }}$

$\mathbf{q}_{\text {error }}$

$\mathbf{q}_{f i x}$

$r_{e}$

$\Lambda_{s t e p, n}$

$\theta$

$\mathbf{v}_{\text {error }}$
Axis of rotation of $\mathbf{q}_{f i x}$

Orthographic projection matrix

The translation error

The ground reaction force underneath the left shoe

The ground reaction force underneath the right shoe

The transformation between the measured and reference $\boldsymbol{\Lambda}_{R L}$

The rotation between the measured and reference $\boldsymbol{\Lambda}_{R L}$

Quaternion rotation used to flatten step in pedestrian tracking algorithm

The rotation error

The transformation from subjects starting location to each step they take

Angle of rotation of $\mathbf{q}_{f i x}$

The translation between the measured and reference $\boldsymbol{\Lambda}_{R L}$ 


\section{Introduction}

This thesis documents the research, design, and experimental characterization of an on-shoe system capable of measuring the relative location and orientation of the shoes. This shoe localization system was created as a part of the BalanceAid project, which aims to create a device to improve the balance of the wearer.

\subsection{Motivation}

Among Canadians who are over 65 years of age, falling is the cause of more than half of all reported injuries and the cause of one fifth of all injury related deaths [1]. For all Canadians, falls are the leading cause of hospitalization due to injury and the leading cause of permanent partial disability [2]. It has been estimated that the annual direct cost of injuries due to falls among Canadian seniors is approximately $\$ 2$ billion, a cost that is projected to increase with time as the number of seniors continues to rise [2]. There is strong evidence of the need for a device capable of improving the postural stability of those with a balance impairment, just as hearing aids have improved the ability to hear for those with a hearing impairment [3]. Additionally, there is a multitude of research showing that balance can be improved by using a method of sensory feedback or substitution $[4,5,6,7,8,9]$. Thus, the BalanceAid project was created by the Advanced Biomechatronics Locomotion (ABL) laboratory at Carleton University 
with the goal of creating a low-cost wearable device capable of improving the balance of the wearer. As will be discussed in Section 1.2.7, there are no widespread commercial devices currently available. Most balance aid devices (typically prototypes created in research labs) work by measuring the tilt and sway of the upper body as a metric of balance, and feed that back to the wearer. The BalanceAid project attempts to improve upon this idea by measuring the wearer's center of pressure $(\mathrm{CoP})$ and/or more advanced dynamic stability measures such as those used in bipedal robotics research. In order to calculate the wearer's CoP, the BalanceAid system must measure the relative location and orientation of the wearer's shoes, thus the motivation for a shoe localization system and this thesis.

Additionally, by choosing to measure the $\mathrm{CoP}$ of the wearer, the BalanceAid device could also function as a wearable gait analysis system. Gait analysis is the measurement and analysis of how an individual walks. Currently, gait analysis is performed using either expensive immobile measurement systems such as force plates and optical marker localization systems, or in a clinic by a trained professional in a way that is difficult to quantify and compare across many subjects [10]. Therefore, there is a desire for a low cost mobile device that works in and out of a laboratory setting that is capable of measuring useful gait data such as the wearer's CoP and the trajectory of the shoes over time.

Finally, while not an aim of the BalanceAid project, another application of an accurate shoe localization system is pedestrian localization. Pedestrian localization, as will be explained further in Section 1.2.3, is locating and tracking people who are travelling by foot. A major difficulty in pedestrian localization is tracking people while moving inside of large buildings, where satellite navigation systems are often unavailable. Because the BalanceAid device measures shoe location and which of the shoes are on the ground, it can be used to track the relative movement of the wearer through a form of dead-reckoning that is potentially more accurate than existing methods. 


\subsection{Background}

This section is intended to provide background material to aid in the understanding of the presented system's motivation, design, and function.

\subsubsection{Balance Aid}

An individual's ability to balance while standing or walking depends on a number of the body's systems working together. A combination of sensory organs give the brain an overall model of how the body is balancing, referred to as equilibrioception. The brain processes this sensory information and then instructs muscles throughout the body to act so that the body remains upright. Problems in any portion of this chain (sensation, processing, or muscle reaction) can result in the loss or reduction of balance $[6,8,5]$. Devices and systems designed to improve balance attempt to either augment the individual's sense of balance or their muscle reaction. The former kind of device is the goal of the BalanceAid project and is described below, while the latter kind of device includes systems such as powered exoskeletons and is not discussed as part of this thesis.

The sensory systems most used in maintaining balance are:

1. the vestibular system (inner ear);

2. the visual system (sight); and

3. proprioception (joint angles and forces).

With current technology, there exist many artificial sensor devices capable of making the same measurements as those made by the human body's sensory organs. Thus, a number of devices have appeared in research and even for sale commercially that use the principle of sensory substitution. Sensory substitution is where the human

body is fed sensory input of one kind (normally from an artificial sensor) through a 
sensory organ traditionally used for another purpose. For example, imagine a device capable of measuring the distance to an object, such as an ultrasonic range finder. The distance measured by the range finder could be used to generate an audible frequency in a pair of headphones, with larger distances creating higher frequencies. If a visually impaired person were to wear the headphones and point the range finder, they would be able to sense the distance to objects in front of them via their sense of hearing. It has been shown that in many sensory substitution combinations the human brain adapts very well to processing the new sensory input given enough training time [11]. Many of the individuals that have diminished balance capability when walking or standing do not lack the mental processing required to balance or the muscle ability to react, but only have a reduced sensory capability. By using sensory substitution, it is possible to augment an individual's deficient equilibrioception with artificial devices, that feed the new information back to the body using a lesser used sensory input path improving their balance.

\subsubsection{Gait Analysis}

Gait analysis is the measurement and study of the way someone walks. Gait analysis is commonly used in sports therapy and, more pertinently, diagnosis and rehabilitation of those with balance disorders. Measurement of a subject's gait parameters is usually made by one or both of the following two methods [10]:

1. In a clinical setting, a professional makes visual observations of a subject walking or of video of them walking. This method is simple but makes it difficult to quantify gait parameters and compare data across many subjects.

2. Using optical tracking and force tracking measurement systems to record the precise pose of the subject's body and their ground reaction forces. This method is very useful and accurate, but makes use of expensive equipment and must 


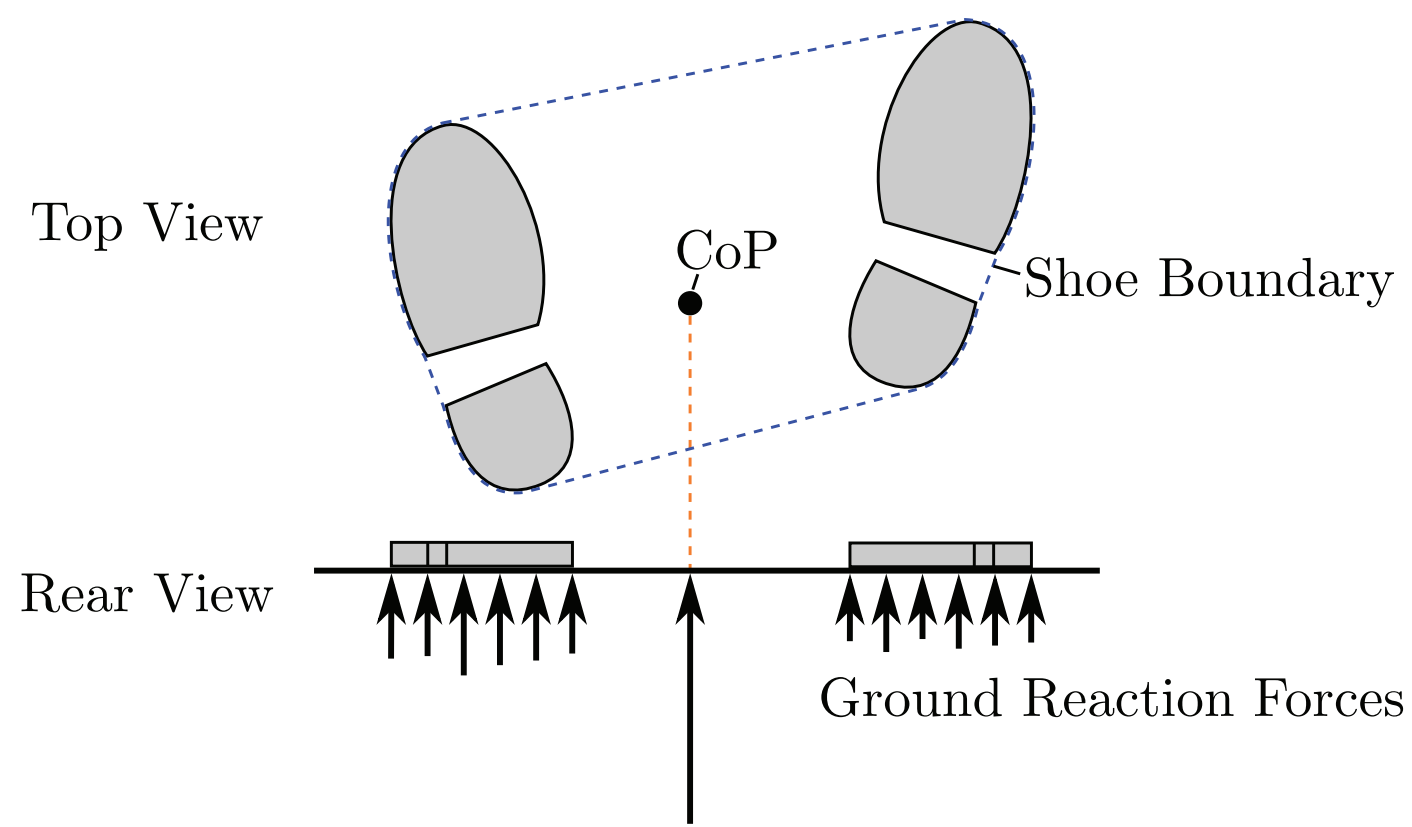

\section{Net Ground Reaction Force}

Figure 1.1.: Depiction of ground reaction forces underneath a pair of shoes, the center of pressure (CoP), and the boundary formed by the two shoes.

take place in a laboratory setting.

\subsubsection{Center of Pressure}

The center of pressure ( $\mathrm{CoP})$ is commonly used as a metric of balance in gait analysis. The $\mathrm{CoP}$ is the location of the net ground reaction force. When standing, the ground applies pressure up against the shoes, as shown in Figure 1.1. The pressure distribution can be replaced with a single force, called the net ground reaction force, acting at a single location called the center of pressure. While standing and walking the pressure distribution underneath the shoes changes causing the CoP to move. Although the CoP will always be within the shoe boundary, as shown in the figure, a CoP closer to the shoe boundary reflects a less stable posture. Even while trying to stand still the $\mathrm{CoP}$ will move and a $\mathrm{CoP}$ with high fluctuations has been linked to an increased chance of falling [12]. 


\subsubsection{Pedestrian Localization}

Pedestrian localization systems are those capable of locating or tracking people, particularly while they are walking. Global navigation satellite systems (GNSS), like the Global Positioning System (GPS), are the preferred method of locating and tracking pedestrians outside of buildings, but the GNSS signals are often too weak to penetrate inside of large buildings. Instead, alternative systems must be employed to locate or track people walking inside of buildings. These systems are commonly called indoor positioning systems (IPS) [13].

One of the systems being studied for use as an IPS is inertial navigation systems (INS). Inertial navigation systems typically function by processing the measurements of accelerometers, rate gyroscopes, and magnetometers to obtain an estimate of the wearer's orientation and acceleration. By mathematically integrating the acceleration of the wearer, the change in position can be calculated. Unfortunately, inertial navigation suffers from a quickly accumulating error in position [14]. The amount of error can often be reduced with more expensive sensors.

When inertial navigation is used with bipedal motion, a method called zero velocity updating can be used [15]. If the inertial sensors are placed on the wearer's shoes, then the fact that a shoe in contact with the ground must have zero velocity can be used to reduce the accumulation of error in position. As a byproduct of placing the inertial sensors in the shoes, an estimate of the shoe's location is also produced.

Another class of systems used in indoor positioning are those that attempt to directly measure the location of the feet, at least when both are planted on the ground. By adding the displacement of each footstep, it is possible to estimate the location of the wearer [16]. 


\subsubsection{Coordinate System and Transformation Notation}

In this thesis, many coordinate systems and transformations are used. This section describes the algebraic notation used with vectors, coordinate systems, quaternions, and transformations. Figure 1.2 depicts the example coordinate systems and vectors referred to in this section.

Coordinate systems (also called frames) and points in space will be denoted with an uppercase roman letter, such as $A$ or $B$. Vectors, which are the displacements from one point to another, will be denoted with a $\overrightarrow{\mathbf{v}}_{X Y}$, which means the vector from $X$ to $Y$. For example, $\overrightarrow{\mathbf{v}}_{A B}$ is the vector from $A$ to $B$. The location of a point in a given coordinate system will be equivalent to saying the vector from the coordinate system's origin to the point.

Vectors can be expressed as a linear combination of the axes of any coordinate system.

For example, $\overrightarrow{\mathbf{v}}_{A B}$ can be expressed using the two coordinate systems $A$ and $B$ :

$$
\overrightarrow{\mathbf{v}}_{A B}=a \hat{\mathbf{x}}_{A}+b \hat{\mathbf{y}}_{A}+c \hat{\mathbf{z}}_{A}=d \hat{\mathbf{x}}_{B}+e \hat{\mathbf{y}}_{B}+f \hat{\mathbf{z}}_{B}
$$

where $\hat{\mathbf{x}}_{A}, \hat{\mathbf{y}}_{A}$, and $\hat{\mathbf{z}}_{A}$ are axes of $A$, and $\hat{\mathbf{x}}_{B}, \hat{\mathbf{y}}_{B}$, and $\hat{\mathbf{z}}_{B}$ are the axes of $B$. The scalars $a, b$, and $c$ are the coordinates of $\overrightarrow{\mathbf{v}}_{A B}$ in frame $A$, while $d, e$, and $f$ are the coordinates of $\overrightarrow{\mathbf{v}}_{A B}$ in frame $B$.

The coordinates of $\overrightarrow{\mathbf{v}}_{A B}$ in any frame can be collected into a convenient $3 \times 1$ column matrix and given their own symbol: 


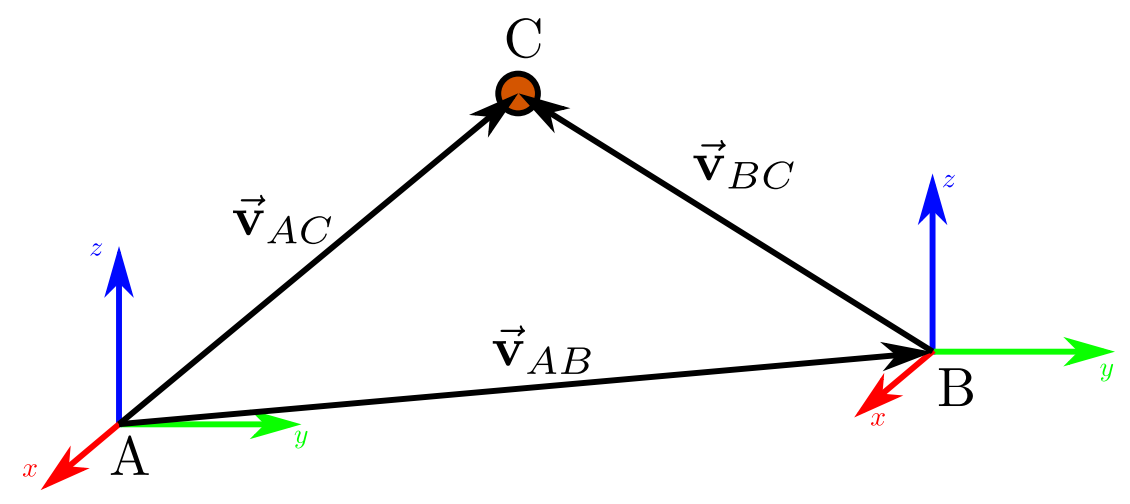

Figure 1.2.: Two coordinate systems $A$ and $B$, and a point $C$, shown with connecting vectors.

$$
\begin{aligned}
& \overrightarrow{\mathbf{v}}_{A B}=\left(\begin{array}{c}
\hat{\mathbf{x}}_{A} \\
\hat{\mathbf{y}}_{A} \\
\hat{\mathbf{z}}_{A}
\end{array}\right)^{T}\left(\begin{array}{l}
a \\
b \\
c
\end{array}\right)=\left(\begin{array}{c}
\hat{\mathbf{x}}_{B} \\
\hat{\mathbf{y}}_{B} \\
\hat{\mathbf{z}}_{B}
\end{array}\right)^{T}\left(\begin{array}{l}
d \\
e \\
f
\end{array}\right) \\
& \overrightarrow{\mathbf{v}}_{A B}=\left(\begin{array}{c}
\hat{\mathbf{x}}_{A} \\
\hat{\mathbf{y}}_{A} \\
\hat{\mathbf{z}}_{A}
\end{array}\right)^{T} \mathbf{v}_{A B, A}=\left(\begin{array}{c}
\hat{\mathbf{x}}_{B} \\
\hat{\mathbf{y}}_{B} \\
\hat{\mathbf{z}}_{B}
\end{array}\right)^{T} \mathbf{v}_{A B, B} .
\end{aligned}
$$

The matrix of coordinates of a vector will use bold letter with no arrow: $\mathbf{v}_{A B, A}$. The subscript means from $A$ to $B$ in frame $A$. In fact, all matrices will use bold letters: column matrices will use lower case letters while larger matrices will use upper case letters. If a vector or its coordinate matrix has a magnitude of one, then it is a unit vector and is denoted with a hat, such as $\hat{\mathbf{v}}_{A B}$ or $\hat{\mathbf{v}}_{A B, A}$. Some common unit vectors are not given a $\hat{\mathbf{v}}$ and are instead given more traditional symbols. For example, the unit axes of a coordinate system will be $\hat{\mathbf{x}}, \hat{\mathbf{y}}$, and $\hat{\mathbf{z}}$; and $\hat{\mathbf{a}}$ is commonly used to mean an axis of rotation.

Unit quaternions[17] will be used for rotations and coordinate system transformations. 
Quaternions are an extension of the notion of complex numbers, but instead of being the sum of a real number and an imaginary number, they are the sum of a real number and three imaginary numbers ${ }^{1}$, and thus contain four elements:

$$
\mathbf{q}=q_{s}+q_{i} \mathbf{i}+q_{j} \mathbf{j}+q_{k} \mathbf{k}
$$

For a unit quaternion, the magnitude, $|\mathbf{q}|=\sqrt{q_{s}^{2}+q_{i}^{2}+q_{j}^{2}+q_{k}^{2}}$, must be equal to one. Conveniently, a quaternion can also be expressed as a real scalar and an imaginary vector part:

$$
\mathbf{q}=\left(\begin{array}{c}
\mathbf{q}_{s} \\
\mathbf{q}_{\overrightarrow{\mathbf{v}}}
\end{array}\right)=\left(\begin{array}{c}
q_{s} \\
q_{i} \mathbf{i}+q_{j} \mathbf{j}+q_{k} \mathbf{k}
\end{array}\right)
$$

Unit quaternions can be easily related to the rotation of an angle, $\theta$, about an axis, â:

$$
\left(\begin{array}{l}
\mathbf{q}_{s} \\
\mathbf{q}_{\overrightarrow{\mathbf{v}}}
\end{array}\right)=\left(\begin{array}{c}
\cos \frac{\theta}{2} \\
\hat{\mathbf{a}} \sin \frac{\theta}{2}
\end{array}\right)
$$

Quaternion multiplication can be denoted using $\otimes$, but it is often left out for simplicity:

$$
\mathbf{q}_{1} \otimes \mathbf{q}_{2}=\mathbf{q}_{1} \mathbf{q}_{2}=\left(\begin{array}{c}
\mathbf{q}_{s, 1} \\
\mathbf{q}_{\overrightarrow{\mathbf{v}}, 1}
\end{array}\right) \otimes\left(\begin{array}{c}
\mathbf{q}_{s, 2} \\
\mathbf{q}_{\overrightarrow{\mathbf{v}}, 2}
\end{array}\right)=\left(\begin{array}{c}
\mathbf{q}_{s, 1} \mathbf{q}_{s, 2}-\mathbf{q}_{\overrightarrow{\mathbf{v}}, 1} \cdot \mathbf{q}_{\overrightarrow{\mathbf{v}}, 2} \\
\mathbf{q}_{s, 1} \mathbf{q}_{\overrightarrow{\mathbf{v}}, 1}+\mathbf{q}_{s, 2} \mathbf{q}_{\overrightarrow{\mathbf{v}}, 2}+\mathbf{q}_{\overrightarrow{\mathbf{v}}, 1} \times \mathbf{q}_{\overrightarrow{\mathbf{v}}, 2}
\end{array}\right)
$$

An asterisk indicates quaternion conjugate, which for unit quaternions is equal to the

\footnotetext{
${ }^{1}$ Complex numbers are often considered to be points in the complex plane, which has one real axis and one imaginary axis. If you imagine extending the $2 \mathrm{D}$ complex plane into a $4 \mathrm{D}$ space by adding two additional imaginary axes orthogonal to the first two, a point in that space would be a quaternion.
} 
inverse:

$$
\mathbf{q}_{A B}^{*}=\mathbf{q}_{A B}^{-1}=\mathbf{q}_{B A}=\left(\begin{array}{c}
\mathbf{q}_{s, A B} \\
-\mathbf{q}_{\overrightarrow{\mathbf{v}}, A B}
\end{array}\right)
$$

where $\mathbf{q}_{A B}$ is the rotation of frame $B$ with respect to frame $A$.

Coordinate system transformations can be performed using quaternion rotations. For example, the transformation of point $C$ from frame $B$ to frame $A$ can be done with:

$$
\mathbf{v}_{A C, A}=\mathbf{v}_{A B, A}+\mathbf{q}_{A B} \mathbf{v}_{B C, B} \mathbf{q}_{A B}^{*},
$$

where:

- $\mathbf{v}_{A C, A}$ is the location of point $C$ in frame $A$;

- $\mathbf{v}_{B C, B}$ is the location of point $C$ in frame $B$;

- $\mathbf{v}_{A B, A}$ and $\mathbf{q}_{A B}$ are the location and orientation of frame $B$ relative to frame $A$; and

- $\mathbf{q}_{A B} \mathbf{v}_{B C, B} \mathbf{q}_{A B}^{*}$ is short-hand for two quaternion multiplications:

$$
\mathbf{q}_{A B} \mathbf{v}_{B C, B} \mathbf{q}_{A B}^{*}=\mathbf{q}_{A B} \otimes\left(\begin{array}{c}
0 \\
\mathbf{v}_{B C, B}
\end{array}\right) \otimes \mathbf{q}_{A B}^{*}=\left(\begin{array}{c}
0 \\
\mathbf{v}_{B C, A}
\end{array}\right) \text {. }
$$

To help simplify the mathematics involving multiple coordinate systems and transformations, a coordinate system transformation will be represented with a $\boldsymbol{\Lambda}$. For example, the coordinate system transformation from frame $B$ to $A$ would be:

$$
\boldsymbol{\Lambda}_{A B}=\left(\mathbf{v}_{A B, A}, \mathbf{q}_{A B}\right)
$$

Using this notation, the transformation shown in Equation 1.9 would be written using function notation: 


$$
\mathbf{v}_{A C, A}=\boldsymbol{\Lambda}_{A B}\left(\mathbf{v}_{B C, B}\right) .
$$

Coordinate systems can also be combined. For example, if there are three frames $D$, $E$, and $F$, and transformations $\boldsymbol{\Lambda}_{D E}$ and $\boldsymbol{\Lambda}_{E F}$ are known, then $\boldsymbol{\Lambda}_{D F}$ can be calculated as:

$$
\begin{aligned}
\boldsymbol{\Lambda}_{D F} & =\boldsymbol{\Lambda}_{D E} \boldsymbol{\Lambda}_{E F} \\
& =\left(\mathbf{v}_{D E}+\mathbf{q}_{D E} \mathbf{v}_{E F} \mathbf{q}_{D E}^{*}, \mathbf{q}_{D E} \mathbf{q}_{E F}\right) .
\end{aligned}
$$

Finally, a coordinate system transformation can also be inverted using:

$$
\boldsymbol{\Lambda}_{A B}^{-1}=\left(-\mathbf{q}_{A B}^{*} \mathbf{v}_{A B} \mathbf{q}_{A B}, \mathbf{q}_{A B}^{*}\right),
$$

such that:

$$
\mathbf{v}_{B C, B}=\Lambda_{A B}^{-1}\left(\mathbf{v}_{A C, A}\right) .
$$

Additional information on quaternions and their use in coordinate system transformations can be found in [17]. A list of symbols used for all coordinate systems as well as other mathematical objects used in this thesis can be found in the Nomenclature section.

\subsubsection{Localization Technology}

The section gives a brief summary of the currently available technology for measuring position and orientation. This information becomes useful in understanding the 
reasons for the system design that will be discussed in Section 2.2.

First, the following is a list of sensors used in localization technology along with a short description:

Accelerometers measure the magnitude and direction of proper acceleration. Proper acceleration is the combination of kinematic acceleration and the acceleration perceived due to gravitational fields.

Gyroscopes measure relative orientation.

Rate gyroscopes measure angular velocity.

Magnetometers measure the magnitude and direction of the local magnetic field.

Inertial measurement units or IMUs are a combination of accelerometers, gyroscopes, and sometimes magnetometers. Together they can estimate orientation with respect to the earth as well as linear acceleration.

Range finders measure the distance to a nearby object by sending out a signal and either measuring the time it takes to be received or the amplitude of the signal when received. These devices can work by sending out acoustic waves, radio waves, or lasers.

Scanning range finders have a mechanism for pointing a range finder in many directions. By scanning through all of the directions it is possible to create a map of distances.

Cameras measure the amount of light coming from many directions. By using an array of light detection sensors, cameras can form an image of the surroundings similar to how people see.

There are a three major approaches to measuring the location of a point at a distance:

1. The simplest case is measuring the direction and the distance to the point simultaneously. While this may seem like the obvious solution, the technology 
required to measure direction and distance is usually cumbersome, expensive, and does not scale down easily to the size required in this thesis. For example, scanning laser range finders are a common option for this method, but are very expensive and in general too large. Cameras could be included in this category, if apparent size of a target is used to estimate its distance from the camera.

2. Trilateration in three dimensions works by making three or four measurements of the distance of the point from different, well known locations. Systems that use trilateration typically work by sending out an electromagnetic (EM) or acoustic signal and measure the time it takes to reach its destination, or by measuring the amplitude of the signal. Both methods can be used to estimate the distance.

3. Triangulation works by making a number of direction measurements to the point from different, well known locations.

Other combinations of distance and direction measurements can also be made to provide a solution.

To measure the relative orientation of two coordinate systems, it is necessary to measure a number of direction vectors in both coordinate systems. In three dimensional space, it is necessary to measure at least two directions vectors. These vectors can be from the environment like the direction to magnetic north, or local to the device itself like the direction from one marker to another.

\subsubsection{Optotrak Certus}

This section introduces a commercial measurement system commonly referred to in this thesis: the Optotrak Certus.

The Optotrak Certus, or simply the Optotrak, is a commercial product for accurately measuring the position of many small markers. The system consists of a central tower, 


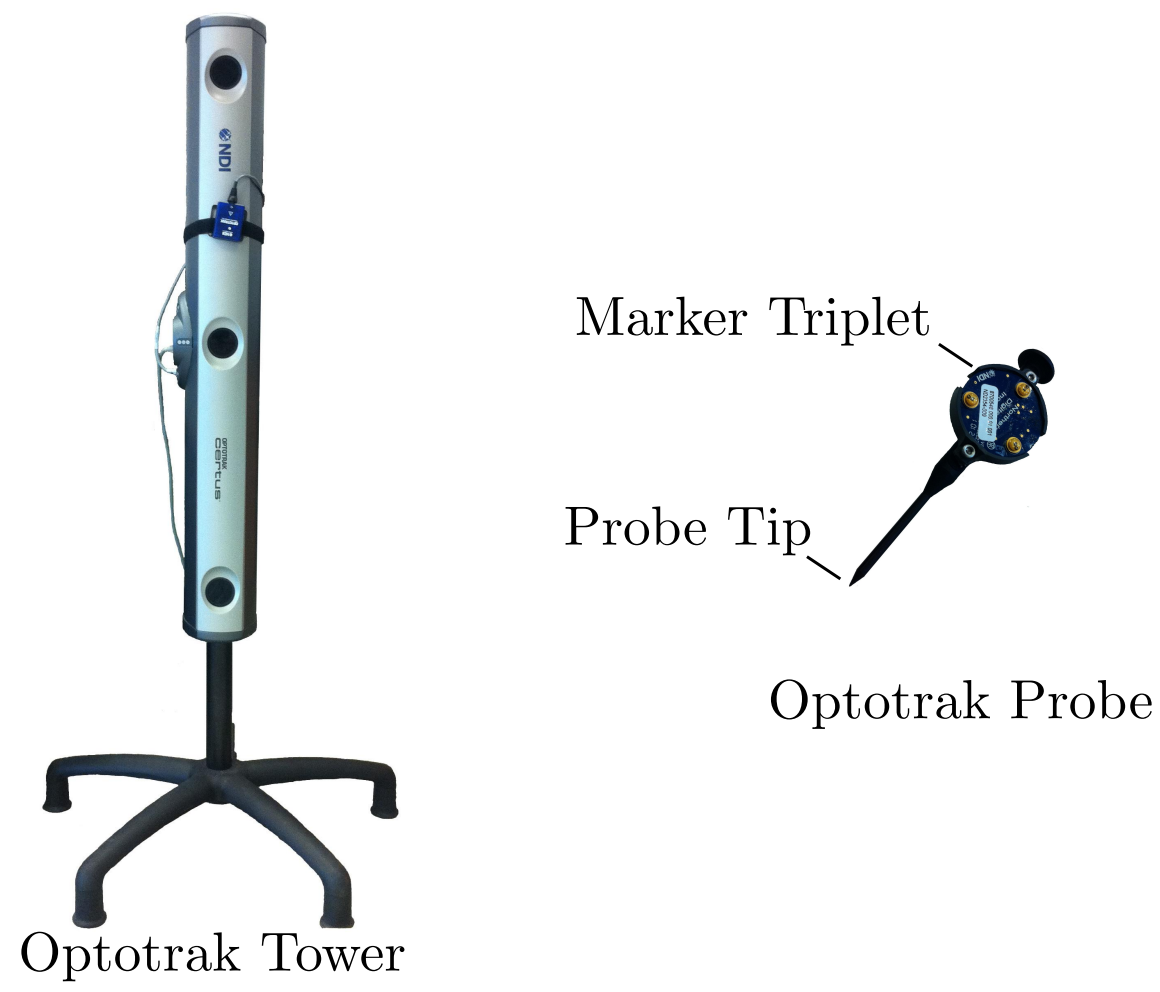

Figure 1.3.: The hardware components of the Optotrak Certus measurement system.

a number of marker triplets, and a probe, all shown in Figure 1.3. The central tower contains cameras that must be able to see the markers to determine their positions. The marker triplets each contain three markers so that the orientation of the marker triplet may be ascertained. The Optotrak probe is simply a marker triplet rigidly fixed to a structure with a sharp point at one end. The sharp point allows the probe to be easily used for determining the precise location of the point on any physical object. The Optotrak is capable of measuring the location of a marker or the probe tip with sub-millimeter accuracy.

The Optotrak system is used in this thesis in Chapters 4 and 5 as part of the calibration and experimentation procedures. To facilitate the use of the Optotrak system for calibration and experimentation, a marker triplet is fixed to the rear of each shoe, as shown in Figure 1.4.

In addition to reporting the position of individual markers, the Optotrak is capable of 


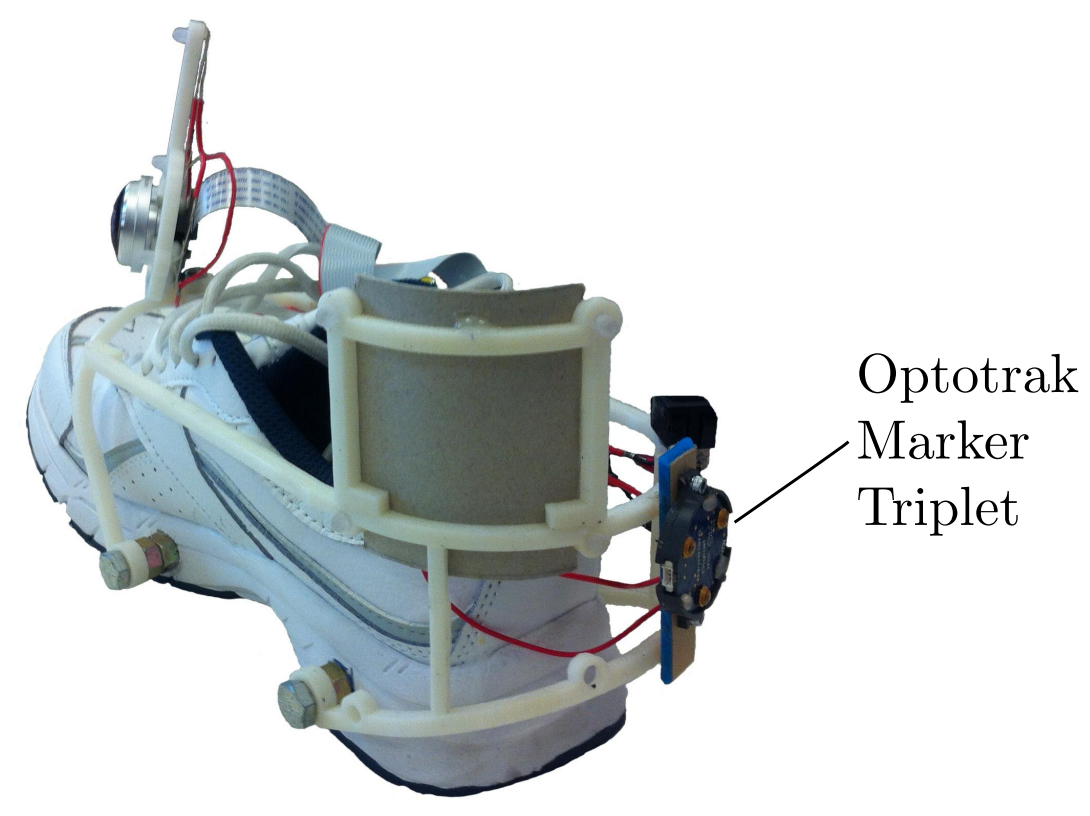

Figure 1.4.: An Optotrak marker triplet located on the rear of each shoe.

reporting the position and orientation of coordinate systems fixed to marker triplets.

\subsubsection{Related Work}

This section summarizes the existing published research related to this thesis. Research done on the localization of shoes, especially using wearable systems, typically comes from three different areas of application: balance aid devices, gait analysis, and pedestrian localization.

\subsubsection{Balance Aid}

There are a number of wearable devices that attempt to aid the wearer in balancing, such as $[4,8,5]$. Most of these devices employ an inertial measurement unit used to measure torso tilt and sway, and a vibrotactile feedback device worn around the waist. A notable exception is [18], where the inertial sensors are on the wearer's head and the tactile feedback mechanism is attached to the tip of the tongue. A significant 
amount of research on balance aid using tactile feedback has come from a group in the Harvard - MIT Division of Health Science and Technology. They have evaluated devices with head mounted and waist mounted inertial sensors coupled with tactile feedback on the torso $[19,20,21]$. They have also performed very informative research into the effects of tactile feedback on the wearer's balance [9, 22, 23].

From these sources, it is concluded that vibrotactile feedback of body inclination can improve postural control, especially in the elderly. Unfortunately, while inertial tilt sensors come in conveniently compact packages and can make measurements at high rates, they have a number of disadvantages:

1. IMUs of sufficient accuracy can be very expensive.

2. Their measurements can be influenced by the other motions of the wearer, such as the linear acceleration or even the rotation rate if the inertial sensors are not close to the center of spin.

3. Measurement of only body inclination or even rate of inclination completely ignores other factors of stability, such as the wearer's stance or grade of the ground.

The measurements from inertial sensors closely resemble the information provided by the vestibular system, but if the intended wearer of the balance aid device is deficient in other sensory systems, then the sensory substitution provided by an IMU alone may not provide the wearer with enough information to maintain postural stability. The goal of the BalanceAid project is to overcome the deficiencies with IMU based balance assistance devices, or to simply provide an alternative technology, by measuring the wearer's center of pressure instead of the body's inclination. 


\subsubsection{Gait Analysis}

In 2003, Fitzpatrick and Kemp investigated the use of shoe mounted cameras for analyzing gait, obstacle detection, and context recognition [24]. They used a single camera and IMU mounted to a shoe, with image processing occurring offline. They concluded that the shoes have good potential as a location for computer vision based devices.

In 2011, Do and Suh presented a system for measuring shoe location and orientation using shoe mounted cameras and externally fixed infrared LED markers [25]. In 2012, they used a camera rigidly attached to the shoe to measure the shoe's position and orientation [26]. While walking, the camera takes images of a strip of markers fixed along the ground. Their system is able to accurately estimate the trajectory of the shoe globally, but only in a very limited setting.

In [27], Edgar et al. presented an on-shoe device capable of recognizing the activity and posture of the wearer. The system uses a three axis accelerometer on each shoe that communicates it measurements wirelessly to a smartphone for processing. The device is an excellent example of an unobtrusive system that can work outside of the laboratory. However, the gait information produced by this system is limited to posture and activity recognition.

The only device found that also attempts to measure the wearer's CoP using onshoe sensors is documented in [10]. Morris' device, called GaitShoe, used an array of force sensors in the sole of the shoe to measure ground reaction forces, and inertial sensors (accelerometer and gyroscopes), electric field sensors, and ultrasonic range sensors to estimate the relative location and orientation of each shoe. The electric field sensors were used to measure the height of the shoe above the ground, and the ultrasonic range sensors were used to calculate the distance between the shoes. While [10] focused mainly on gait analysis, it also contained some work on real-time sensory 
feedback. The GaitShoe device relies on the integration of inertial measurements and makes the assumption that the shoes move with zero vertical translation and only pitch rotation. While this is sufficient for estimating the location of the shoe while in contact with the ground, it does not provide a true three dimensional trajectory of the shoe over time.

In later work, Bamberg et al. use similarly instrumented shoes to perform research into further gait analysis [28, 29, 30, 31], sensory feedback [32], and slip detection [33].

From these previous developments, it is concluded that existing on-shoe gait analysis systems either lack the freedom of being able to be used in everyday settings, or they do not actually measure the complete position and orientation of the shoes over time. The BalanceAid device aims to provide very detailed information, such as the complete trajectory of the shoes though space as well as the pressure distribution underneath each shoe, while still being able to be worn in everyday settings.

\subsubsection{Pedestrian Localization}

There are a large number of shoe mounted inertial navigation systems [14, 13, 34]. Of particular note is [15], where Foxlin presented the idea of the zero-velocity position updates as pseudo-measurements to an extended Kalman filter when both feet are on the ground as part of his NavShoe system. Foxlin's system significantly reduced the error of inertial dead-reckoning systems when used in bipedal motion.

Yuan and Chen have a system where each lower limb has an IMU to determine orientation of that limb, making it possible to determine pose and location and orientation of each foot [35]. While their system successfully tracks the wearer, including the locations of the shoes, the use of many accurate IMUs results in a costly system.

The only pedestrian localization system found that uses on-shoe cameras to measure 
relative location and orientation was done very recently: In 2013, Placer and Kovačič demonstrated a pedestrian localization system that makes use of a camera mounted on one shoe looking at a marker on the other [36]. The camera is only able to measure the position of the other shoe when the right foot is forward, and uses inertial measurements and an unscented Kalman filter (UKF) to estimate the position in between measurements. All the calculations, including image processing, were done offline.

While it is not the primary goal of the BalanceAid project, it is hoped that the technology developed will provide an useful measurement for pedestrian tracking applications.

\subsection{BalanceAid Project Overview}

The BalanceAid project was created by the ABL laboratory with the aim of developing a device capable of improving the balance, or reducing the chance of falling, of those with balance related dysfunction. The requirements of the device are the following:

1. Should be wearable, with no external infrastructure;

2. Should not hinder the normal movement of the wearer;

3. Should be able to work in realistic settings outside of the laboratory; and

4. Should work for various sources of balance disorders.

The BalanceAid system works by feeding supplementary sensory information to the wearer, providing them with an improved sense of balance. In order to be useful for many people with various balance disorders, the system does not attempt to replace a single sensory organ, such as the vestibular system or foot pressure, but instead measures the wearer's center of pressure (CoP), a useful high level metric of balance. In order to measure the wearer's $\mathrm{CoP}$, it is necessary to measure the ground reaction 


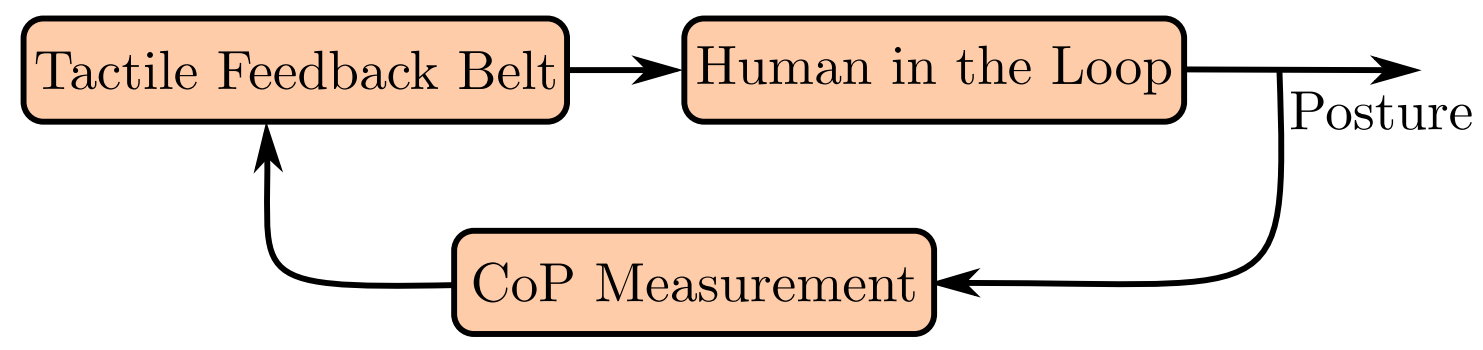

Figure 1.5.: Block diagram description of feedback of center of pressure information.

forces underneath each shoe, as well as the relative location and orientation of each shoe. Once the $\mathrm{CoP}$ is calculated, that information is fed back to wearer via a belt with embedded vibrotactile actuators, or tactors. This feedback process is shown in the block diagram in Figure 1.5.

The BalanceAid system is broken up into three different subsystems:

1. A system for measuring the relative location and orientation of the shoes. This system is the topic of this thesis, and will be described in much more detail.

2. A system for measuring the ground reaction forces underneath each shoe. The design system of this system was completed by Patricia Giacoman Zarzar in parallel with the localization system and is documented in [37].

3. A system for informing the wearer in real-time of the calculated CoP (ie, the vibrotactile belt). At the time of writing, this system is still under development by the ABL laboratory.

\subsection{Localization System Overview}

This section describes the localization system. It contains a brief overview of the system and an explanation of its basic principle of operation.

Figure 1.6 shows a high-level overview of the localization system. The system includes two typical shoes. Attached to the exterior of each shoe is a camera, eight markers 


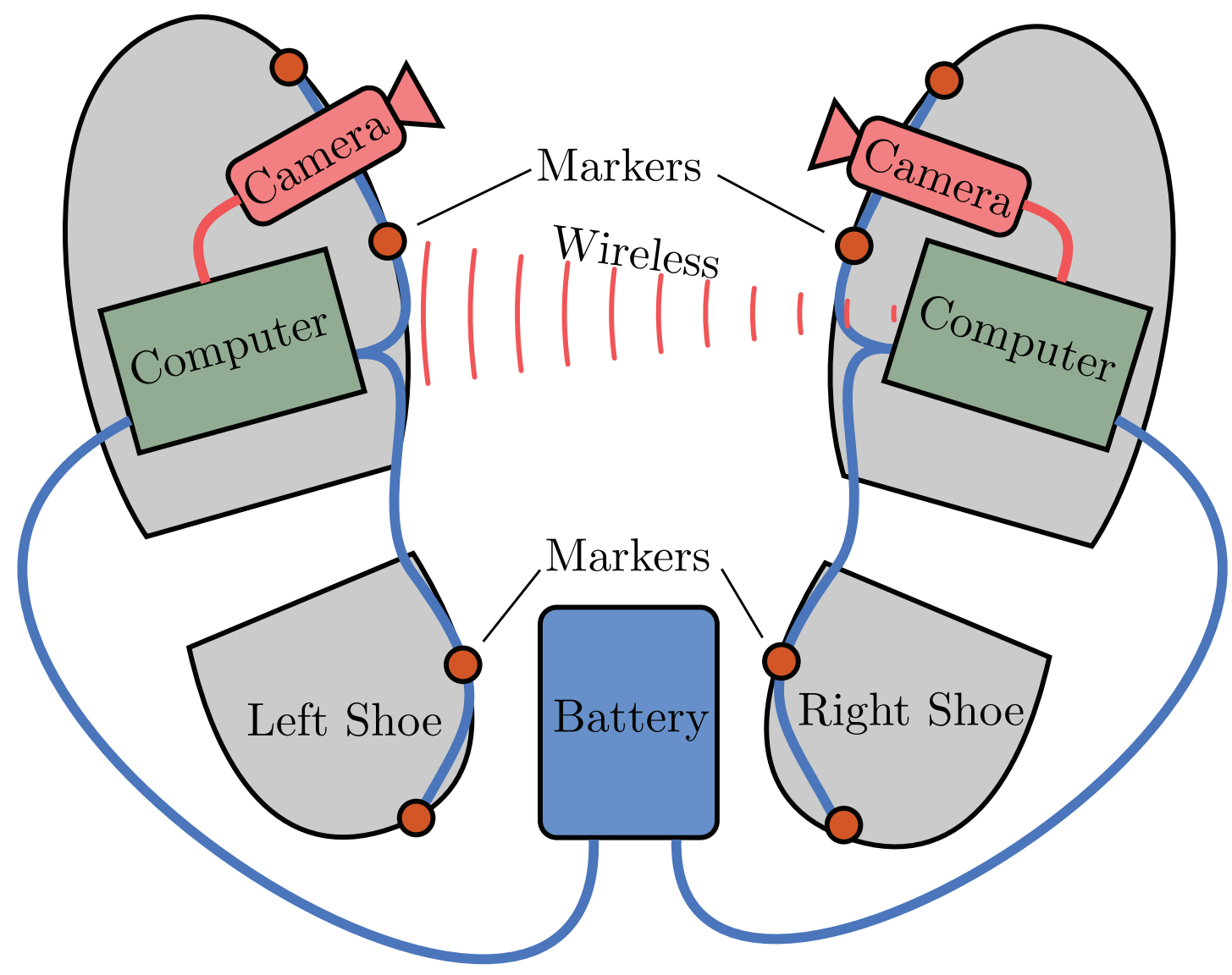

Figure 1.6.: Overview of the localization system design.

(only four shown), a small computer, wiring, and a structure to hold it all together. The cameras and markers on the shoes allow each computer to calculate the relative position and orientation of the shoes. The images captured by the cameras are processed in real-time by the small computer on each shoe, with the resulting data eventually being combined together on the left shoe via a wireless connection.

The final data products (i.e., the position and orientation of the shoes) are logged in the left shoe's memory where they can be downloaded for later analysis, or they can be sent wirelessly to another computer in real-time.

The design of the system can be divided into two major components: the hardware design and the software design. The hardware design consists of the choice and 
placement of the physical parts of the system such as the camera, lens, electronics, and the shoe itself. The design of the system's hardware is described in detail in Chapter 2. The software design consists of all the steps in processing the data fed into the on-board computer such as the algorithms and inter-process communication. The design of the system's software is documented in Chapter 3.

\subsubsection{Principle of Operation}

Simply put, the relative position and orientation of the shoes is determined in the following way: Each camera captures images of the other shoe. Each captured image will also contain the markers fixed to the shoe. From the location of each marker set in the image, it is possible to calculate its direction away from the camera using a camera model. Once the directions to four markers are known, the distances to those markers can be calculated using a solution to the perspective-4-point problem. Finally, with the positions of the four markers known with respect to both shoes, it is possible to calculate an optimal coordinate system transformation from one shoe to the other. The coordinate system transformation contains the relative location and orientation of the shoes.

\subsection{Contributions}

The contributions made by this thesis are summarized as follows:

1. The first design of a system capable of calculating the relative position and orientation of the wearer's shoes using on-board cameras, markers, and image processing was developed.

2. A self-contained prototype of the system was implemented using commercially available parts and a custom-made structure and image processing software. A 
calibration procedure for the prototype was developed and performed.

3. A custom-made real-time embedded software architecture was developed for the localization system as well as for the BalanceAid project, including components for hardware access and inter-process communication.

4. As part of the BalanceAid project, the localization system and force sensing insole system were integrated.

5. Experiments using the prototype were performed using 6 healthy subjects to characterize the performance of the system.

\subsection{Thesis Overview}

\section{Chapter 2: Hardware Design}

This chapter describes the hardware used in the localization system and details the reasons for the selection and configuration.

\section{Chapter 3: Software Design}

This chapter gives a description of the software used in the localization system. This include both the system architecture, interprocess communication, and the image and data processing algorithms.

\section{Chapter 4: Calibration}

This chapter explains the experiments and data processing performed to calibrate the system.

\section{Chapter 5: Tests \& Characterization}

This chapter describes the setup, processing, and results of the experiments used to test and characterize the system.

\section{Chapter 6: Conclusion}


This chapter summarizes the accomplishments of this thesis, possible improvements to the design or implementation, and recommendations for future work. 


\section{Hardware Design}

This chapter describes the hardware used in the localization system and details the reasons for the hardware's selection and configuration.

\subsection{Overview}

As can be seen in Figure 2.1, the system consists of two shoes with the following attached to the exterior of each:

- a Raspberry Pi Model B single board computer (Section 2.3);

- a camera with a fish-eye lens (Section 2.4); and

- eight infrared (IR) light emitting diode (LED) markers (Section 2.5).

These components are attached to the shoe with a 3D-printed skeletal structure which is fixed to the sole of the shoe (Section 2.6). The skeletal structure allows the components to be placed in their ideal locations while being largely unaffected by the deformation of the shoe during walking. It also allows the whole system to be transferred to a different pair of shoes if necessary.

The Raspberry Pi and markers on both shoes are powered by an external battery pack, which can clip on to the wearer's belt or pants. The battery pack is connected to each shoe by a long USB cable that is strapped to the leg. 


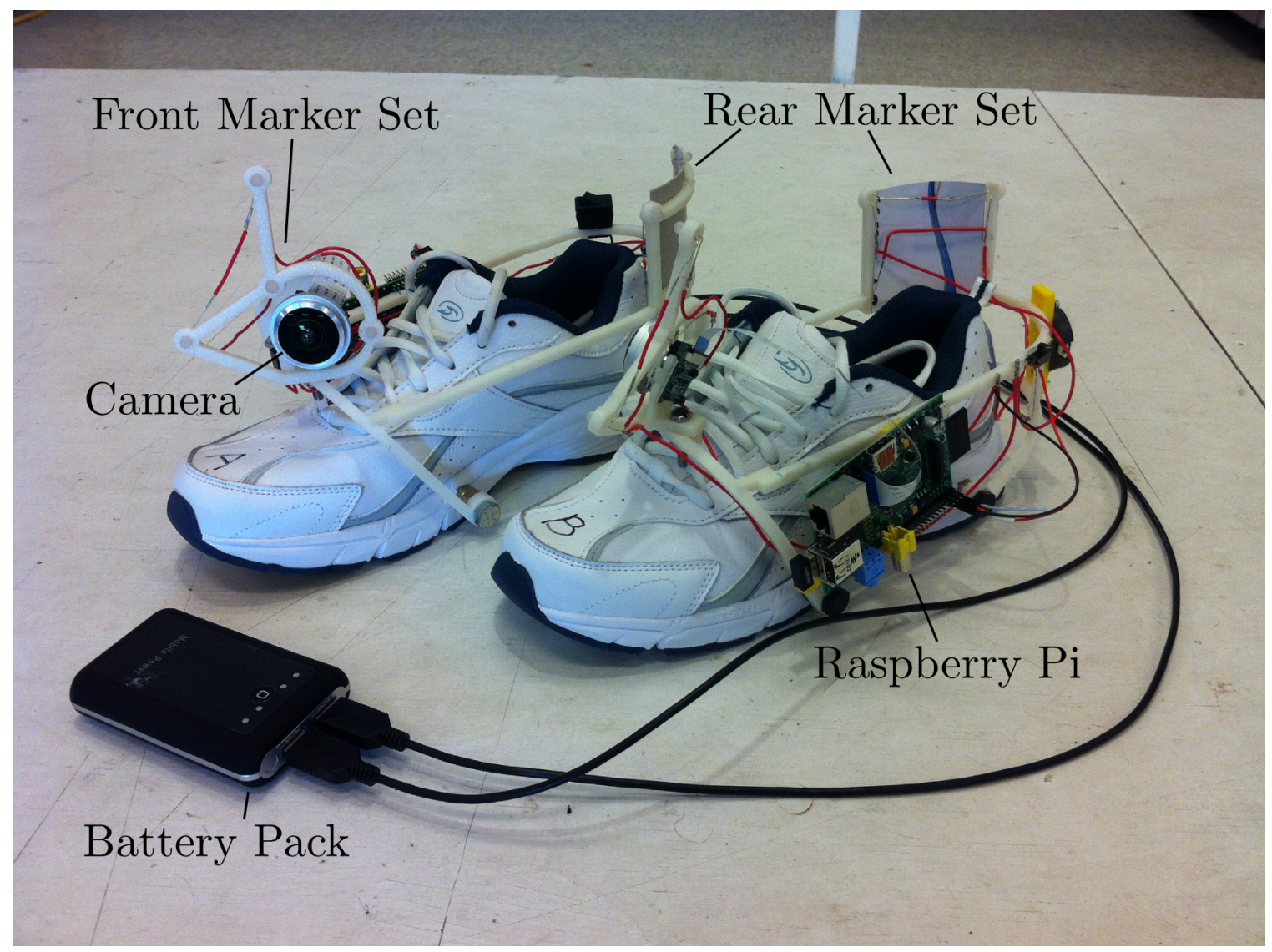

Figure 2.1.: Hardware assembly used in localization system.

\subsection{Design Considerations}

In addition to the BalanceAid requirements, stated in Section 1.3, the localization system has the following design requirements:

1. Should share hardware resources with the other subsystems of the BalanceAid project to minimize the total system size;

2. Should work for normal walking movement;

3. Should attempt to minimize cost; and

4. Should attempt to maximize accuracy.

There is little research on what would be a necessary accuracy for balance aid purposes. An accuracy on the order of 1 or 2 centimeters would be advisable goal based 
on the size of the shoes and distanced involved.

The hardware for measuring the location and orientation of the shoes could be located anywhere on the body, but a shoe mounted system was chosen for the following reasons:

1. The system for measuring ground reaction forces would certainly have hardware on the shoes already. It is advantageous to locate the two system close by to facilitate the sharing of computing resources. Additionally, it is a long term goal of the BalanceAid project to compact the system enough to fit everything on the shoes.

2. A camera system requires line of sight to both shoes, and there are few locations on the body where a single camera could be placed where it would always see both shoes and not interfere with the wearer's movement.

The battery was placed off-shoe as its weight was deemed too high for comfortable walking. Two separate smaller batteries could be placed on each shoe for shorter duration periods of operation.

As was discussed in Section 1.2.5, there are many possible principles by which the position and orientation of the shoes could be measured. It was decided that the use of a camera sensor with easily recognizable markers would best achieve the requirements for the following reasons:

1. EM or acoustic based trilateration lacked the desired accuracy.

2. Scanning range finders would be too large to wear on a shoe.

3. IMUs alone can be used to estimate the orientation of the shoes, but not their relative position, thus two separate sensor systems would be required for position and orientation, increasing cost and complexity. Low cost IMUs might be an appropriate addition to the system in further work. 
A typical camera based triangulation system uses two cameras looking at the same point in space (stereoscopic vision). But the hardware required to processes the images from two cameras simultaneously is not yet compact enough to fit on a single shoe. Instead this localization system uses a single camera looking at a set of markers rigidly fixed to the other shoe.

\subsubsection{Design Limitations}

The limitations of this design are:

1. Because of hardware limitations, the sampling rate is only $15 \mathrm{~Hz}$. This means that during quick strides, only a few measurements may be made. A different selection of hardware should be able to achieve $30 \mathrm{~Hz}$ or even $60 \mathrm{~Hz}$, but it was estimated that the time required to integrate such hardware would take longer than was available.

2. As will be discussed in Chapter 3 - Software Design, the exposure of the camera is quite low at 400 microseconds. But with any non-zero exposure, the markers will streak in the image when moved quickly. If the streaks are long enough that they intersect each other, it can become very difficult to make a measurement. Thus this limits the speed of motion that the system can measure, but from tests performed with the prototype, streaking does not appear to be prevalent during speeds encountered during fast walking.

3. Because of the limited field of vision of the cameras, there are certain placements of the feet that cannot be measured or will provide incorrect measurement, such as with feet crossed or with a large rotational offset between the two feet. Fortunately, none of these cases are expected for normal walking.

4. The camera requires line of sight to some of the markers, obstructions between the shoes could result in incorrect measurements. 


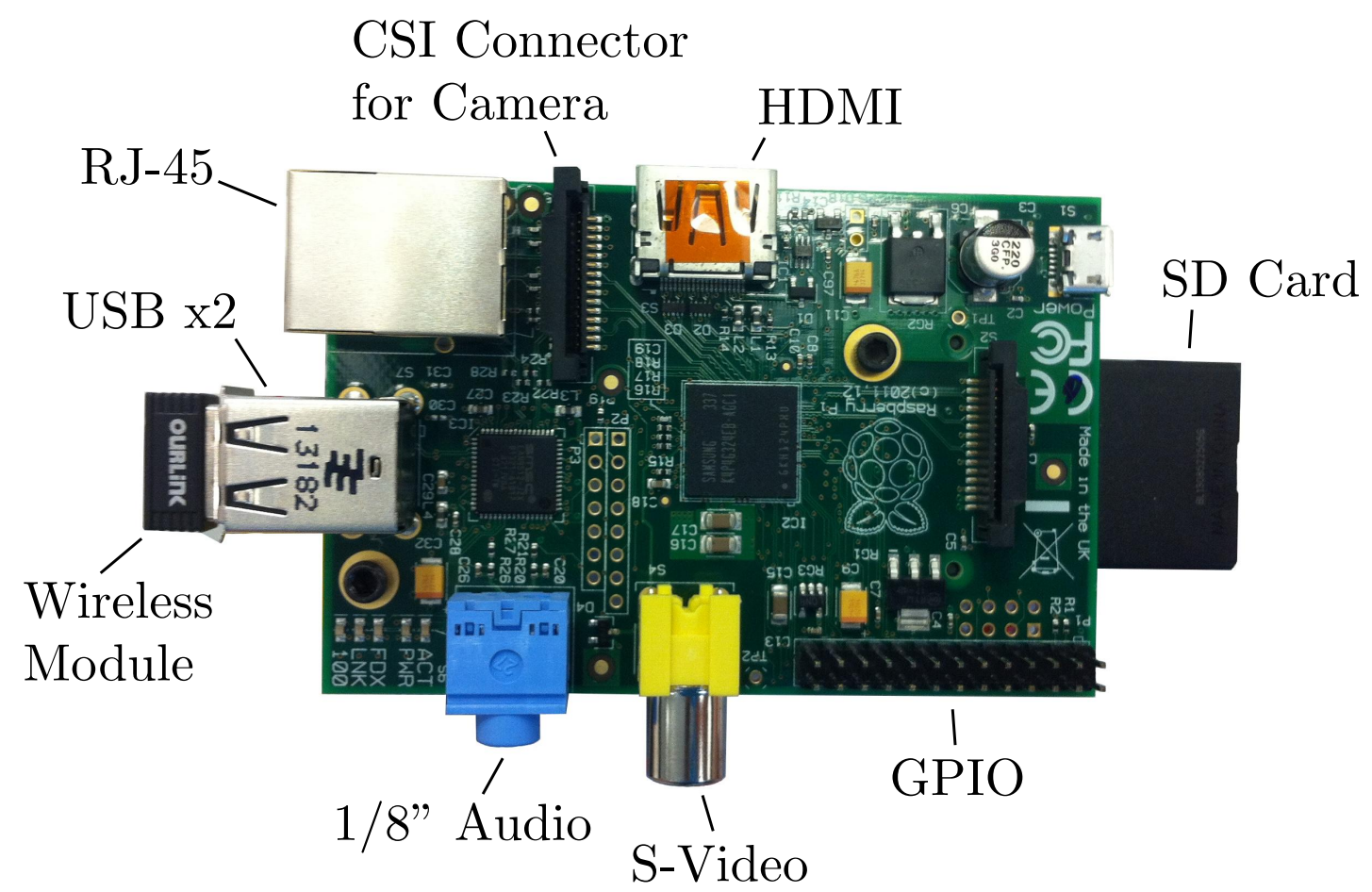

Figure 2.2.: Raspberry Pi Model B single board computer with labels.

5. The location of the camera and markers above the shoe is not very structurally robust.

6. Currently, the design will mistake the sun for an infrared marker. Further work is needed to allow use outside during sunny weather.

\subsection{Raspberry Pi}

The Raspberry Pi Model B, shown in Figure 2.2, is a small, low-cost, single-board computer made by the Raspberry Pi Foundation, aimed at the education or hobbyist market [38]. The relevant technical specifications of the Model B are shown in Table 2.1. 
The Raspberry Pi was selected for this system (specifically the prototype) for its low cost, small size, ease of use, and because it contained the necessary input/output capability required. Of particular importance was the Raspberry Pi's ability to handle the image processing necessary for this system to function. This is largely possible because of the on-board graphics processing unit (GPU) which handles the incoming camera images, and the high processing speed (for computers of this size) of the central processing unit (CPU). While the power consumption is not very low, it is low enough that the single battery pack can power both Raspberry Pis for almost two hours of continuous operation.

The Raspberry Pi Model B has two universal serial bus (USB) ports for peripheral devices. During operation, one of the USB ports is used for a small wireless module (shown in Figure 2.2) allowing the Raspberry Pis to communicate wirelessly to each other using the $802.11 \mathrm{~g}$ specification, or to any other wireless devices for monitoring and control. For the purpose of testing and development, the Raspberry Pis connect to an external wireless access point, but they could also form an ad-hoc wireless network, removing the necessity of the wireless access point. The second USB port and the high-definition multimedia interface (HDMI) port are used during development for connecting a keyboard and monitor directly to the Raspberry Pi.

Finally, the camera serial interface (CSI) connector is used to connect the Camera Module, the GPIO pin headers are used to supply power to the markers, and the

Table 2.1.: Selected technical specifications of the Raspberry Pi Model B [39].

\begin{tabular}{cc}
\hline CPU & $700 \mathrm{MHz}$ ARM11 ARM1176JZF-S core \\
\hline GPU & Broadcom VideoCore IV \\
\hline RAM & $512 \mathrm{MiB}($ Shared with GPU $)$ \\
\hline Power Consumption & $3.5 \mathrm{~W}(700 \mathrm{~mA}$ at $5 \mathrm{~V})$ \\
\hline Size & $85.0 \times 56.0 \times 17 \mathrm{~mm}$ \\
\hline Weight & $40 \mathrm{~g}$ \\
\hline
\end{tabular}




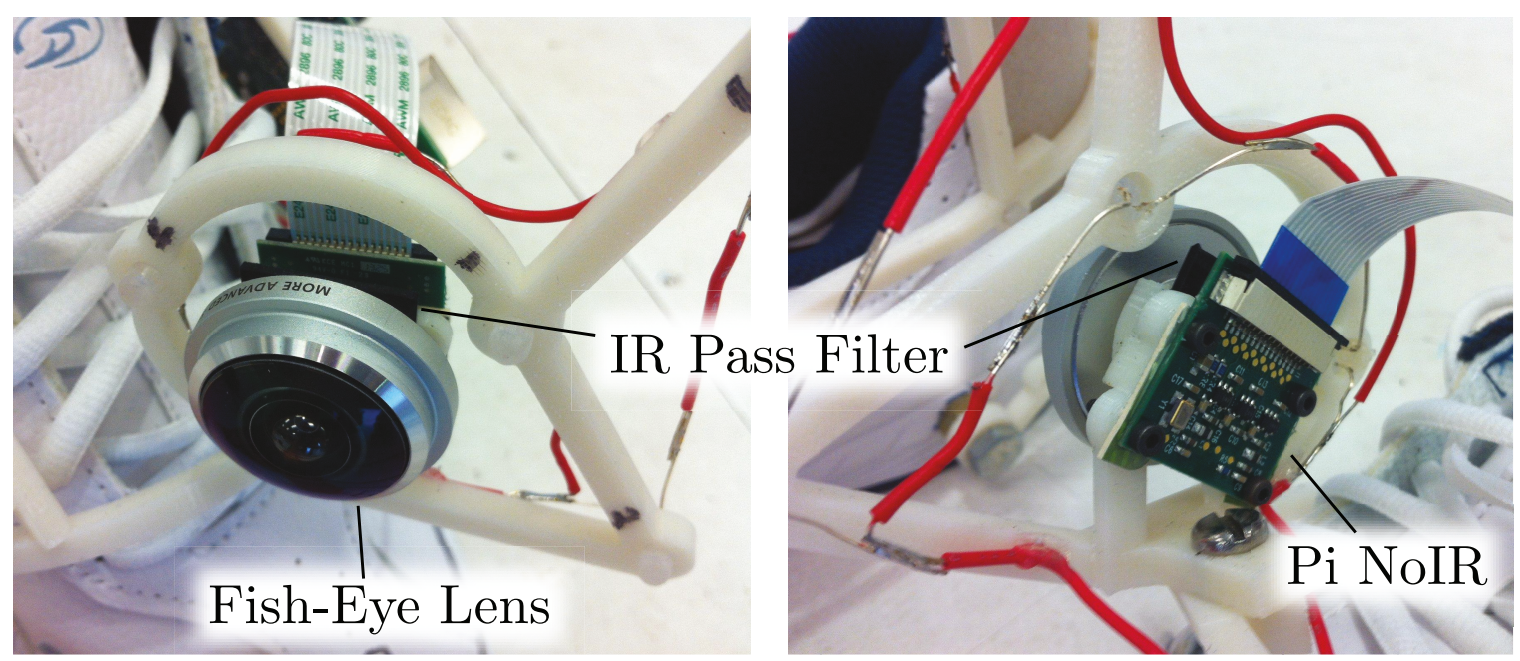

Figure 2.3.: The camera assembly supported on each shoe. The assembly is composed of a Pi NoIR Camera Board, an IR pass filter, a fish-eye lens, and the mounting structure.

micro-USB connector is used to receive power from the battery pack. Each Raspberry $\mathrm{Pi}$ is bolted directly to the skeletal structure using its two mounting holes.

\subsection{Camera Board, Lens, and Filter}

The core sensor for the localization process is the camera. Each shoe supports a camera assembly consisting of a Pi NoIR camera module, an IR pass filter, a fish-eye lens, and the 3d printed mounting structure, all shown in Figure 2.3.

The Pi NoIR camera module is a small 5 Mega-pixel CMOS sensor and breakout board made specifically for the Raspberry $\mathrm{Pi}$ [40]. It is a variant of the standard Raspberry $\mathrm{Pi}$ camera module, in that it is sensitive to light in the near infrared spectrum in addition to visible. Table 2.2 shows the relevant technical parameters of the camera module. The camera module communicates with the Raspberry Pi via its CSI connector. Because of this, it is capable of transferring much larger images at higher rates than a USB connected camera.

Placed in front of the camera module is a superfisheye lens made by Photojojo [42]. 
This lens provides the camera with a field of view (FoV) of greater than 180 degrees $^{1}$, significantly increasing how much the camera module can see alone. This fish-eye lens was chosen because of its small size, low cost, large field of view, and because its projected image fit well on the camera module's sensor area. It is only recently that lenses this small and cheap have become available, driven by the mobile phone accessory market. Among available lenses of similar size and cost, the field of view on this lens is unique. Finally, the sensor size of the camera module, commonly referred to as $1 / 4$ inch, is quite small and the number of fish-eye lenses designed to fit the sensor area was limited.

In between the camera module and the fish-eye lens is a thin IR pass filter, made by Hammond Manufacturing. The camera sensor is sensitive to both visible and near-infrared light, but the markers emit only in infrared. The IR pass filter is used to block all sources of visible light that might otherwise interfere with the image processing by allowing only the infrared wavelengths to reach the sensor.

A single camera viewing four IR markers is sufficient to calculate the location and orientation of the other shoe. Given the range of motion of the shoes and the obstructions caused by the wearer's shoes and legs, it is difficult to obtain a camera/marker

\footnotetext{
${ }^{1}$ The actual FoV with this camera is difficult to state accurately, because of the distortion at the extreme angles. The manufacturer's claim is 235 degrees. Additionally, the circle of projection is cropped in some areas by the small sensor size, meaning the maximum view angle from the optical axis depends on the azimuthal angle around the optical axis.
}

Table 2.2.: Selected technical parameters of the Pi NoIR camera module [41].

\begin{tabular}{cc}
\hline Sensor type & OmniVision OV5647[4] Color CMOS QSXGA \\
\hline Sensor size & $3.67 \times 2.74 \mathrm{~mm}$ \\
\hline Pixel Count & $2592 \times 1944$ \\
\hline Pixel Size & $1.4 \times 1.4 \mathrm{um}$ \\
\hline Field of View & $54 \times 41$ degrees (without fish-eye lens) \\
\hline Board size & $25 \times 24 \mathrm{~mm}$ \\
\hline
\end{tabular}




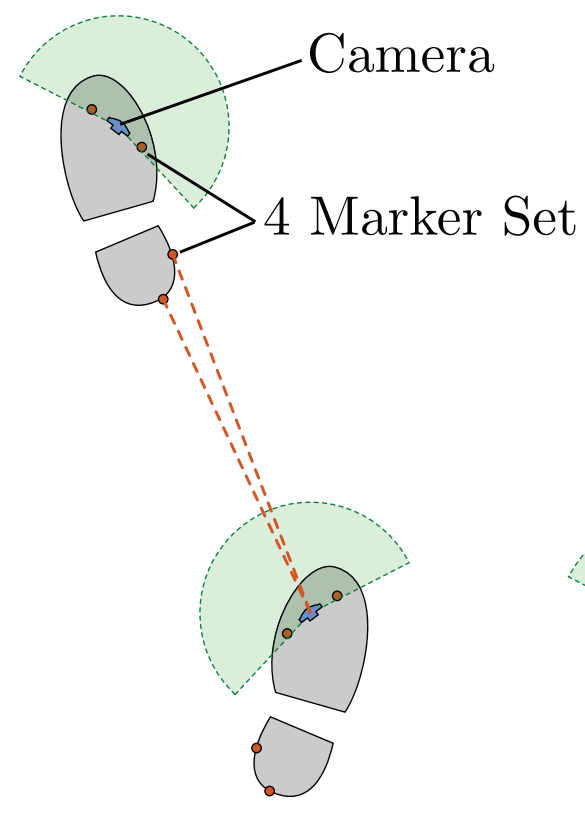

Left Shoe Forward

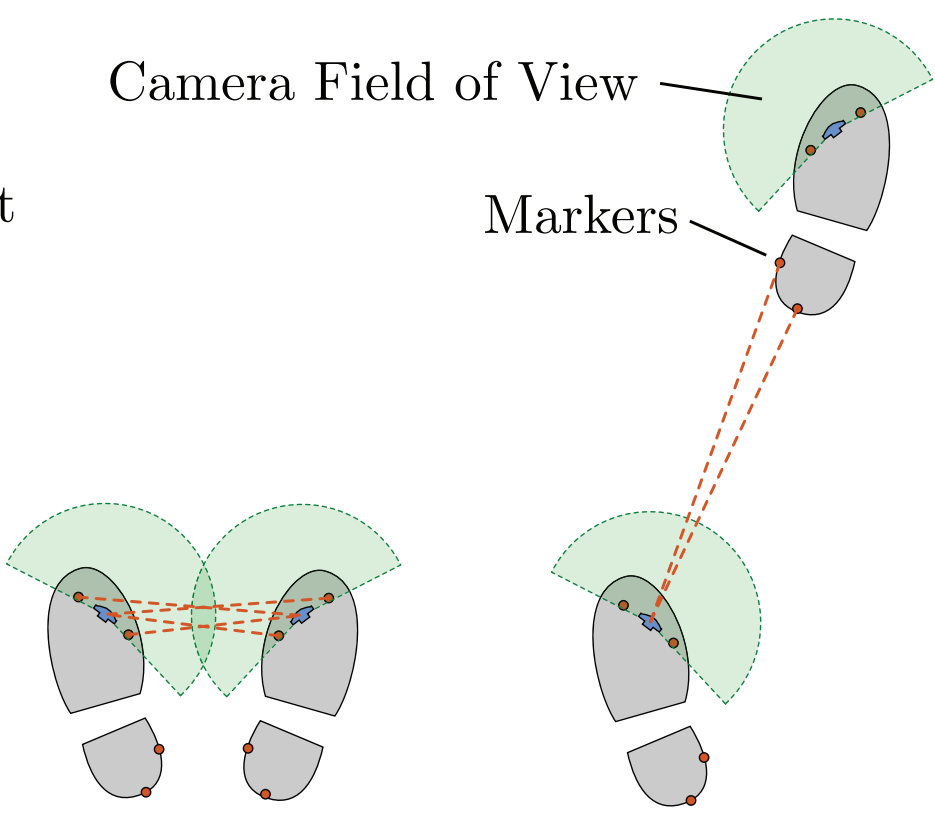

Standing

\section{Right Shoe Forward}

Figure 2.4.: Field of vision of each camera during different stages of walking.

configuration in which a single camera and four markers can be placed on the shoes so that they are always in view for typical walking movement, even with the large field-of-view provided by the super fish-eye lens. The configuration chosen for this system is eight markers and one camera on each shoe. The cameras are pointed so that together they cover a much wider range of motion than just one can. Figure 2.4 shows the fixed orientation of the cameras on each shoe. When in the nominal standing position, both cameras can see the opposing markers and two solutions are calculated and averaged. During long steps, only one camera will be able to see a marker set and derive a solution.

Having each shoe capable of calculating its own solution provides a simple form of redundancy in case one shoe cannot form a solution. If both solutions are calculated they are averaged together providing an increase in accuracy.

With the current placement of cameras and markers, the markers will appear closer 


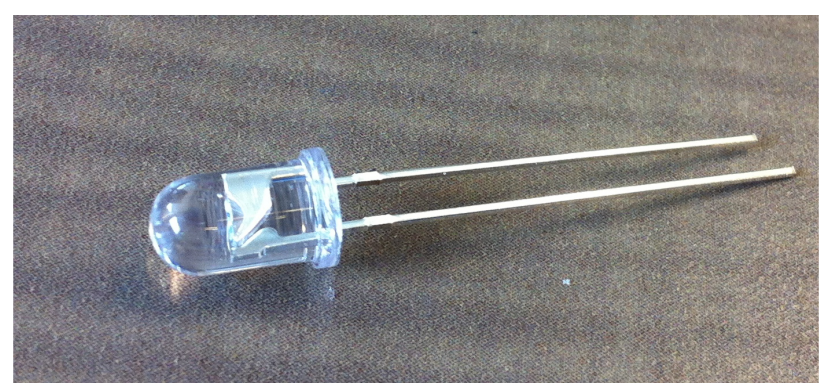

Figure 2.5.: Infrared LED used as a marker in the localization system.

to the center of the field of view of the camera when long strides are taken, as shown in Figure 2.4. Because of the distortion effects of the fish-eye lens discussed in Section 3.2.4, this will yield more accurate and less noisy results.

\subsection{IR Markers}

As mentioned in the system overview (Section 1.4.1), the localization system works by processing the images from a camera pointed at the opposing foot to determine its position and orientation. While it would be possible to take regular images of the shoe, run a shoe recognition algorithm and compare that with an internal model of the shoe to determine its position and orientation, that would take more processing power than is available on board the Raspberry Pi to happen in real-time. Instead, easily identifiable infrared LED markers are used. As will be discussed in detail in Section 3.2.5, by determining where four markers are in the image and on the other shoe, it is possible to determine the other shoe's position and orientation.

Each marker is a typical LED (5 mm diameter), as shown in Figure 2.5. But instead of producing visible light, these LEDs produce light in the near-infrared spectrum $(850 \mathrm{~nm})$. Infrared light is invisible to the human retina, but is visible to most camera technologies. Using infrared markers allows the use of very bright markers (very visible to cameras), that go unnoticed by the shoes wearer or others. 


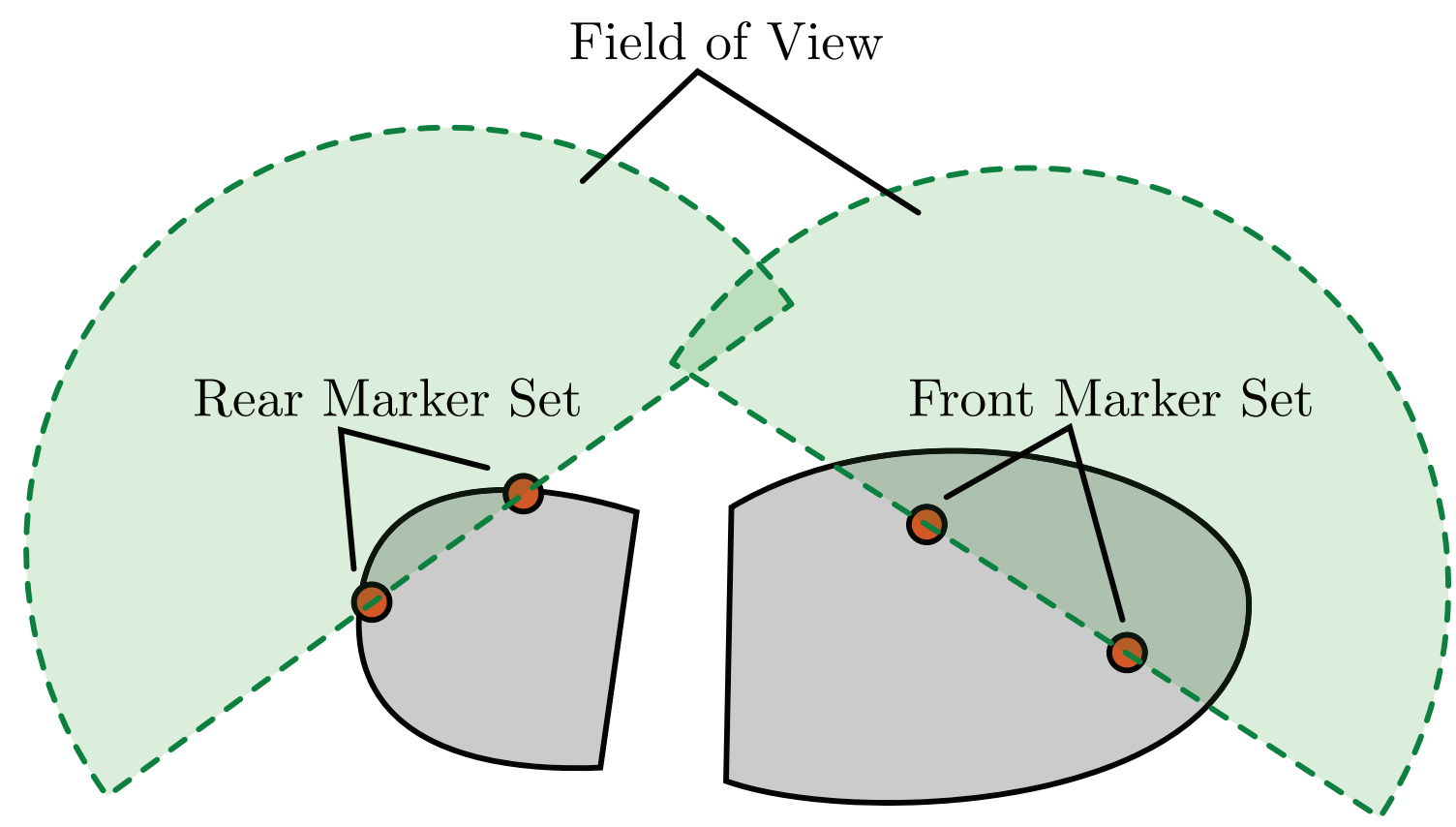

Figure 2.6.: Visibility of the two marker sets. Field of view in this case refers to the volume in which each marker set would be visible.

While only four markers are necessary for a localization solution to be calculated, there is no location on the shoe that a set of four markers would remain visible to the camera at all times while walking. Instead, two sets of four markers are used: One designed to be visible from the rear and one visible from the front with some overlap visibility from the side, as shown in Figure 2.6. Additionally, each set of four markers is set in a two dimensional plane to simplify the calculations during processing as described in Section 3.2.5. The two marker set must be rigidly fixed to one another in the current data processing scheme. With the addition of a bend sensor in the shoe's sole or insole it might be possible to measure the transformation between the two marker sets in real time and remove the need for the rigid connection.

The $5 \mathrm{~mm}$ LEDs were initially selected because they are easily commercially available through many commercial outlets, and to ensure they would remain visible in the image even at the extreme distances. The disadvantage of such large LEDs is that the center point of the LED, which is used in the localization calculations, is harder to 
determine from different view angles and in the presence of partial obstructions. It is recommended that for future devices, smaller LEDs are investigated to overcome this disadvantage. A marker set using surface mounted LEDs would be easier to construct and would necessarily place the LEDs in a plane, which is a requirement of the later calculations.

Finally, to increase marker visibility, the surfaces of the LEDs are sanded rough causing the emitted light to be more diffuse.

\subsection{Shoe and Structure}

The shoes used in the prototype are women's size 8 (US) Dr. Scholl's running shoes, but in principle any shoe can be used. The skeletal support structure was 3D printed using ABS plastic. Four threaded nuts are glued to the outside edge of the shoe's sole and the skeletal structure is bolted to those nuts, as shown in Figure 2.7.

For the localization system to function correctly and accurately, the camera, the markers, and the shoe itself must not move relative to each other during operation. Unfortunately, while walking, a typical soft shoe can deform significantly, particularly at the toe. The skeletal structure attempts to minimize relative movement between these components in two ways:

1. By separating the camera and the markers from the surface of the shoe it allows the shoe to deform comfortably for the wearer while not affecting the components directly.

2. By mounting to the rear of the sole (which remains relatively undeformed while walking), the structure remains largely unaffected by the large toe bending deformation at the front of the shoe.

Figure 2.7 shows the skeletal structure with and without the deformation from the 

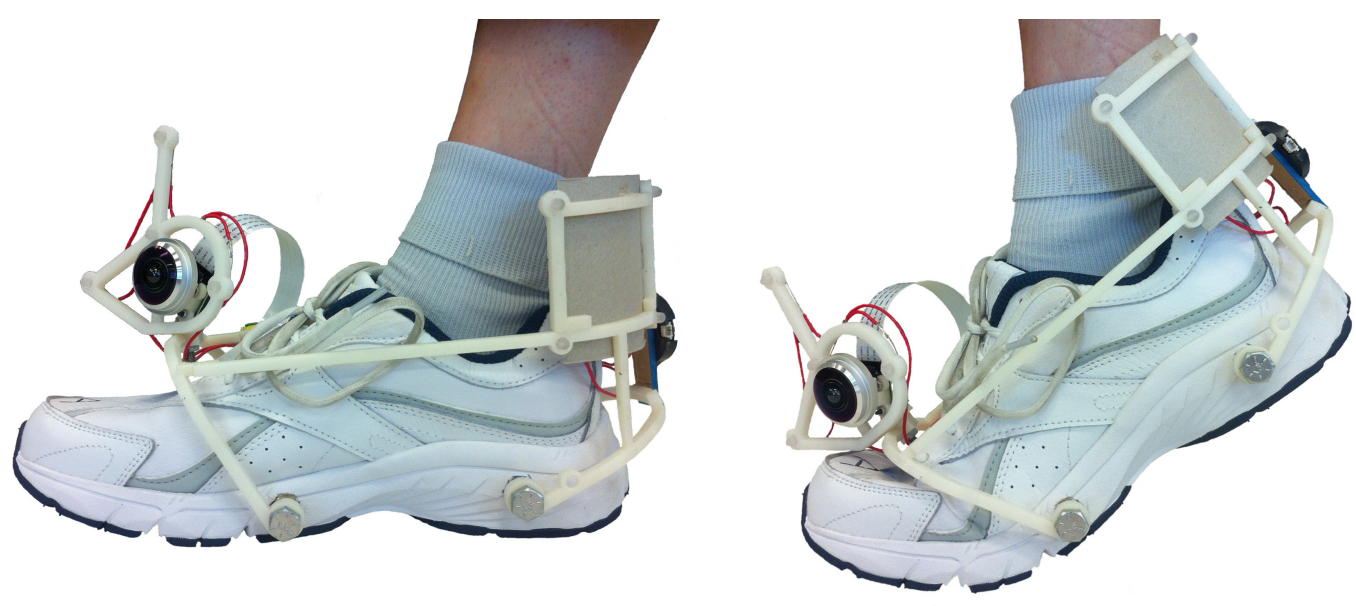

Figure 2.7.: Skeletal support structure shown with and without shoe deformation.

toe bending typically seen during walking.

In addition to providing rigidity, the skeletal structure provides the required geometry for optimal marker and camera placement, as discussed in Sections 2.5 and 2.4, which is not naturally created by the surface of the shoe. Additionally, mounting everything on a skeletal structure has the added benefit of being easily removable from the shoe should the need arise.

The main challenge of the structural design is the placement of the camera and markers so that at least one camera on one shoe can see a set of markers on the other shoe at all times. The current design achieves this geometric requirement, but the resulting structure is very susceptible to deformation, is fragile, and can be cumbersome to the wearer. It is recommended that in the next design iteration a new camera and marker set placement be chosen to overcome these shortcomings. A design that only requires one marker set for each shoe would be preferable as it would allow the entire structure to become more compact as well as have benefits in the image processing described in Chapter 3. To achieve the geometric constraint mentioned, it may be necessary to depart from the on-shoe model and consider mounting the camera elsewhere on the body, such as the legs looking at both feet. 


\subsection{Chapter Summary}

The hardware on each shoe of the localization system consists of

1. a Raspberry Pi Model B single board computer;

2. an Adafruit Industries miniature WiFi module;

3. a Sandisk 16 GiB SD memory card;

4. a Raspberry Pi NoIR camera module;

5. a Superjojo Super fish eye lens;

6. eight infrared LEDs (5 mm diameter);

7. 5 V Battery pack;

8. cables/wiring; and

9. a 3D printed skeletal structure.

As is shown later in Chapter 5 , the camera/marker concept for calculating the position and orientation of the shoe works successfully, but there are a number of recommendation for improvement on the design which will be highlighted here:

1. It is recommended that smaller LED markers are used, perhaps even surface mounted LEDs.

2. The camera sensor should be sized to include the entire circle of projection of the fish-eye lens.

3. The on-board computer and camera module should be selected to achieve a higher framerate, such as 30 frames per second. It may be possible to stay with the Raspberry $\mathrm{Pi}$, as long as the lens/sensor are sized differently.

4. A new camera and marker set placement should be chosen to significantly reduce the fragility and complexity of the current skeletal structure. It may be 
necessary to depart from the on-shoe model and mount the camera elsewhere on the body such as the legs so that both feet are visible. 


\section{Software Design}

This chapter describes the algorithms and software used in the localization system.

\subsection{Overview}

The objective of the localization system software is to capture images from the camera and determine the localization solution, which is defined below.

Installed on each Raspberry Pis is a GNU/Linux operating system [43]. During operation, each operating system runs a process called shoevision and one runs another process called the integrator.

Each shoevision process is responsible for capturing images from the camera, processing those images, and calculating a localization solution, if possible, and sending its results to the integrator. The shoevision processes on each shoe are identical, except that each is compiled with different calibration parameters, as well as slightly different instructions reflecting that the shoes are mirror images.

The integrator receives the solutions from the two instances of shoevision, combines and filters them using a Butterworth filter and logs the resulting localization solution. ${ }^{1}$ Figure 3.1 shows the flow of information through the system software.

\footnotetext{
${ }^{1}$ In the larger BalanceAid system, the integrator also receives the pressure insole data, calculates the center of pressure, and controls the tactile feedback.
} 


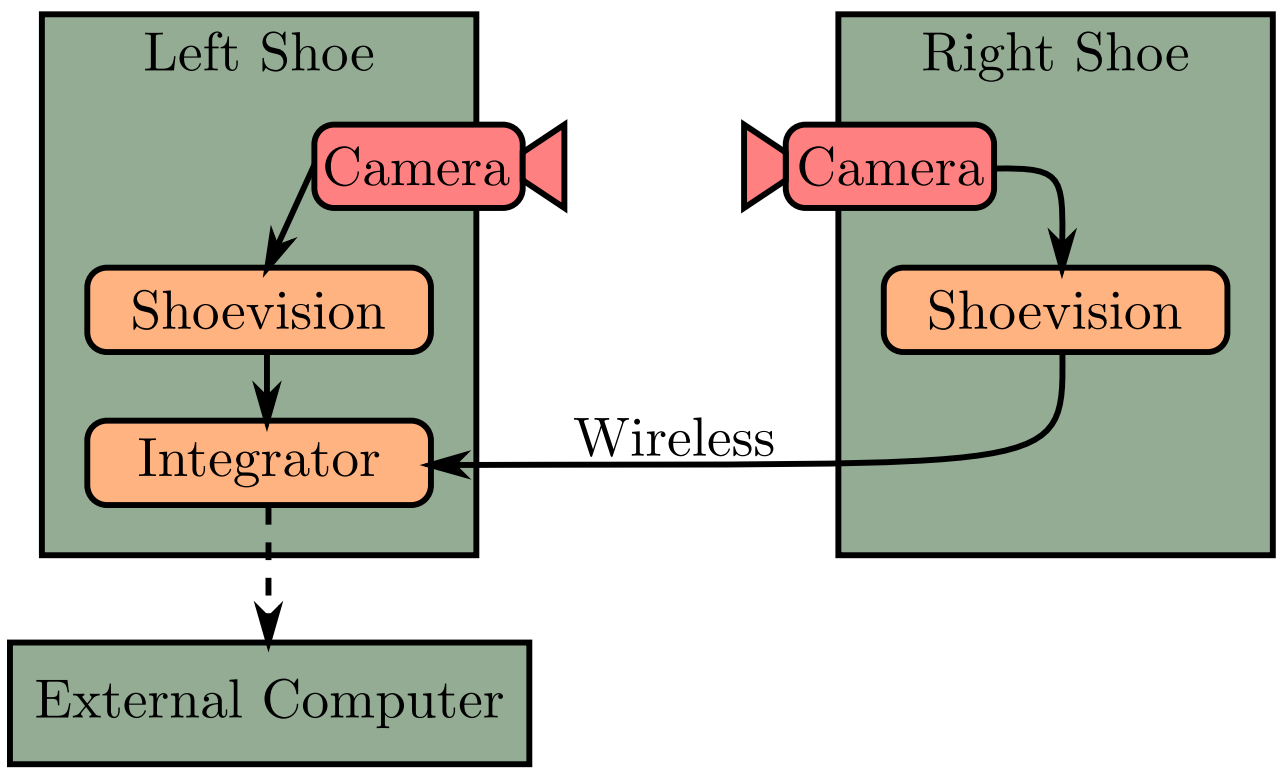

Figure 3.1.: Overview of software architecture. Communication between the processes is using the transmission control protocol (TCP). Communication between the shoes is wireless $(802.11 \mathrm{~g})$.

Fixed to each shoe is a coordinate system, as shown in Figure 3.2. Both coordinate systems, referred to as Camera Frame $L$ for the left shoe and Camera Frame $R$ for the right shoe, are centered and aligned with their shoe's camera. The exact alignment will be further described in Section 3.2.4. The localization solution is the coordinate system transformation from Camera Frame $L$ to Camera Frame $R$ :

$$
\boldsymbol{\Lambda}_{R L}=\left(\mathbf{v}_{R L, R}, \mathbf{q}_{R L}\right)
$$

The calculation of $\boldsymbol{\Lambda}_{R L}$ described in the remainder of this chapter assumes the images are captured on the right shoe and the visible markers are on the left shoe. When the solution is calculated on the left shoe, the procedure is identical but the final solution must be inverted to calculate $\boldsymbol{\Lambda}_{R L}$, instead of $\boldsymbol{\Lambda}_{L R}$. 


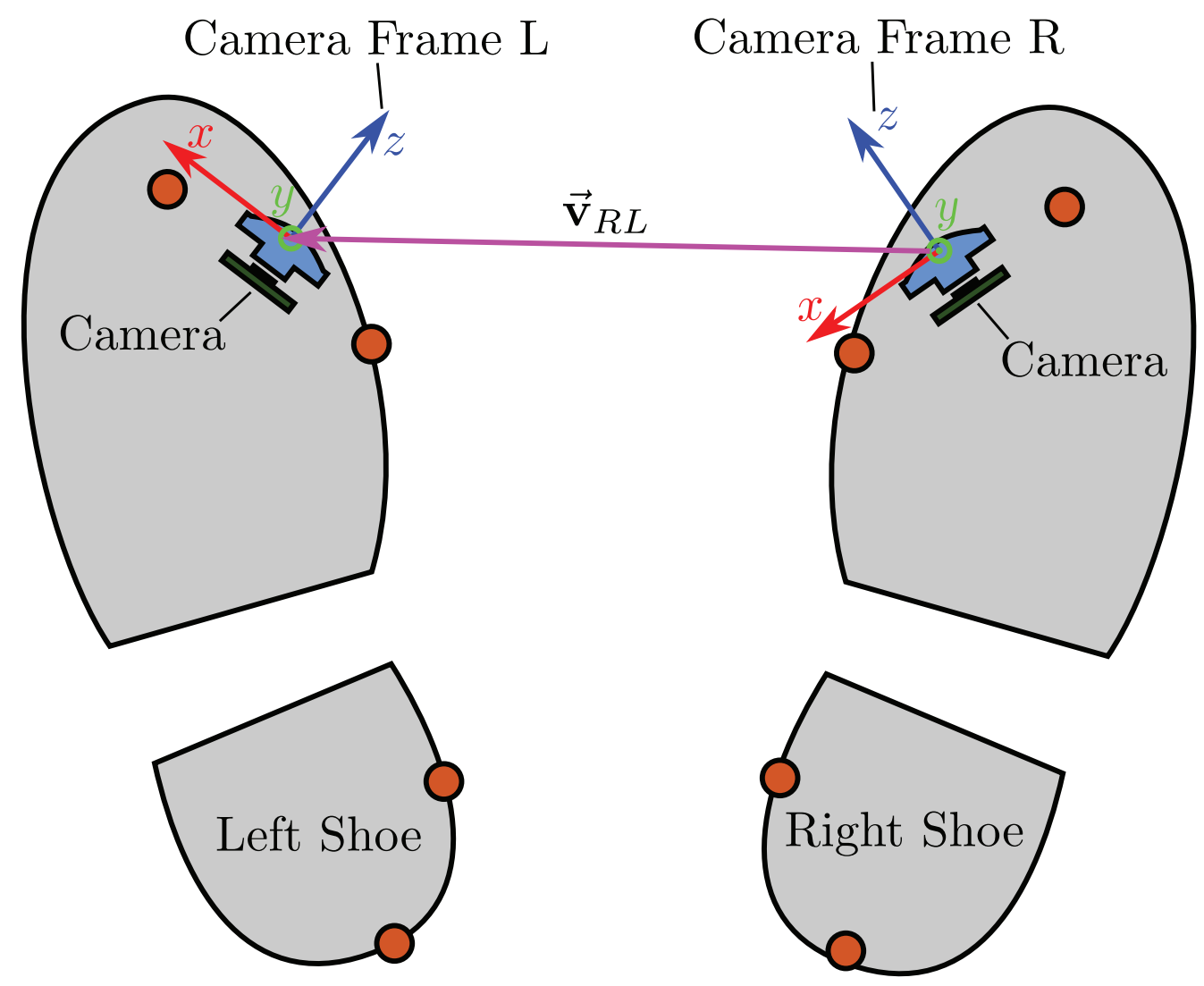

Figure 3.2.: Geometric depiction of the localization solution.

\subsection{The Shoevision Process}

The shoevision process consists of two threads operating simultaneously:

1. The main thread initializes the camera for image capture, spawns the second thread and waits for the user to end the process. When the user ends the program, it then handles the clean up (this ends the processing thread as well).

2. The processing thread waits for captured images from the camera. When an image is received, it performs the following operations:

a) connected component labeling (CCL) on the image produces a list of blobs (Section 3.2.1);

b) the resulting blobs are filtered (Section 3.2.2); 


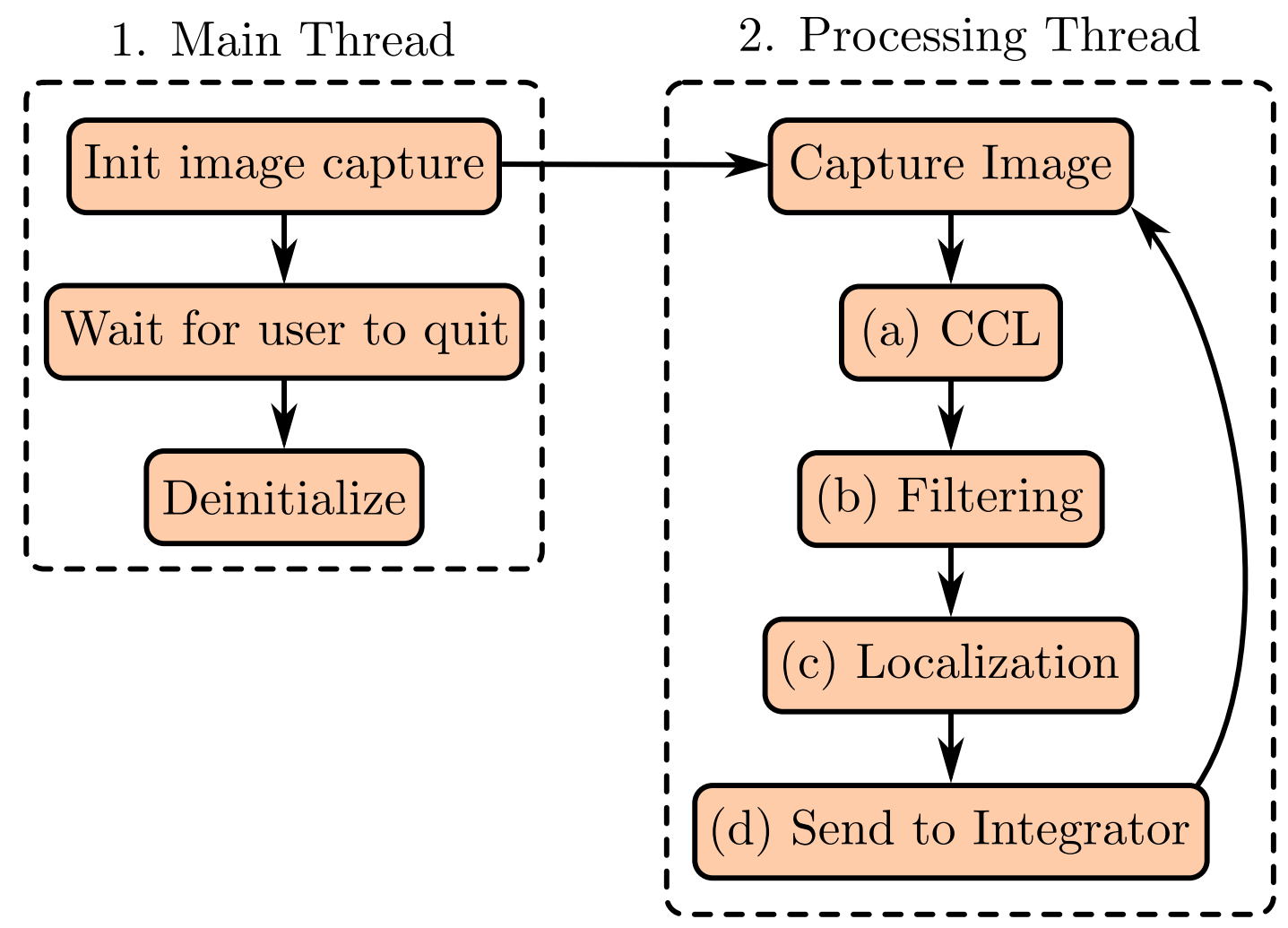

Figure 3.3.: Overview of the shoevision process.

c) the FIND_SOLUTION algorithm uses the filtered blobs to calculate a localization solution (Section 3.2.3); and

d) the solution is sent to the integrator.

\subsubsection{Connected Component Labelling}

The first step after receiving an image from the camera is to find where the markers appear in the captured image. Specifically, the marker's $\mathrm{x}$ and y coordinates in the image and the markers apparent size are desired. This step is accomplished with a simple connected component labelling algorithm.

Connected component labelling (also known as blob extraction) is the algorithmic process of finding the regions of contiguous pixels within an image that share similar properties, such as colour. A digital image is typically composed of a rectangular grid 
of small elements called pixels. Each pixel has only a single colour and brightness. When the camera module captures an image on the Raspberry Pi, each pixel is represented by three bytes (YUV420 pixel encoding), two for the colour and one for the brightness. Since the camera sensor is only receiving a single wavelength of infrared light, the entire image is a single colour and only varies in brightness. Thus, before any processing, all of the colour information is discarded. This leaves each pixel only having a single byte representing brightness. A single byte expressed as an integer represents a range of 0-255. In this section, only a black and white image is desired. White pixels will be those with an integer value of 190 and above, while black pixels will be those less than 190. This operation is commonly referred to as thresholding the image.

The image captured uses the camera's full resolution and thus has a size of 2592 by 1944 pixels. The camera captures 15 images every second, exposing the sensor for 400 microseconds during each capture. Due to the IR pass filter in the camera assembly (Section 2.4), the bright IR LED markers, and the very low exposure time, the captured images are largely black with the markers appearing as distinct white dots, as shown in Figure 3.4. The camera's focal length is approximately $30 \mathrm{~cm}$ (About half a typical stride length). At a focal length this close to the camera, the depth of field is very short. That is, there is a only a small region where objects will appear very clear in the image. Away from the focal length, the LED markers do appear slightly blurrier, but since only the center point of the markers needs to be found, it does not cause much error. Additionally, at such a low exposure the streaking due to the dynamic motion of the LEDs was found to be small.

Each IR marker creates a region of connected white pixels separated by black pixels. These connected regions are called connected components or simply blobs. For clarity, the term marker will now only be used when referring to the LEDs themselves, while blobs will refer to the appearance of the markers in the image. This includes properties 


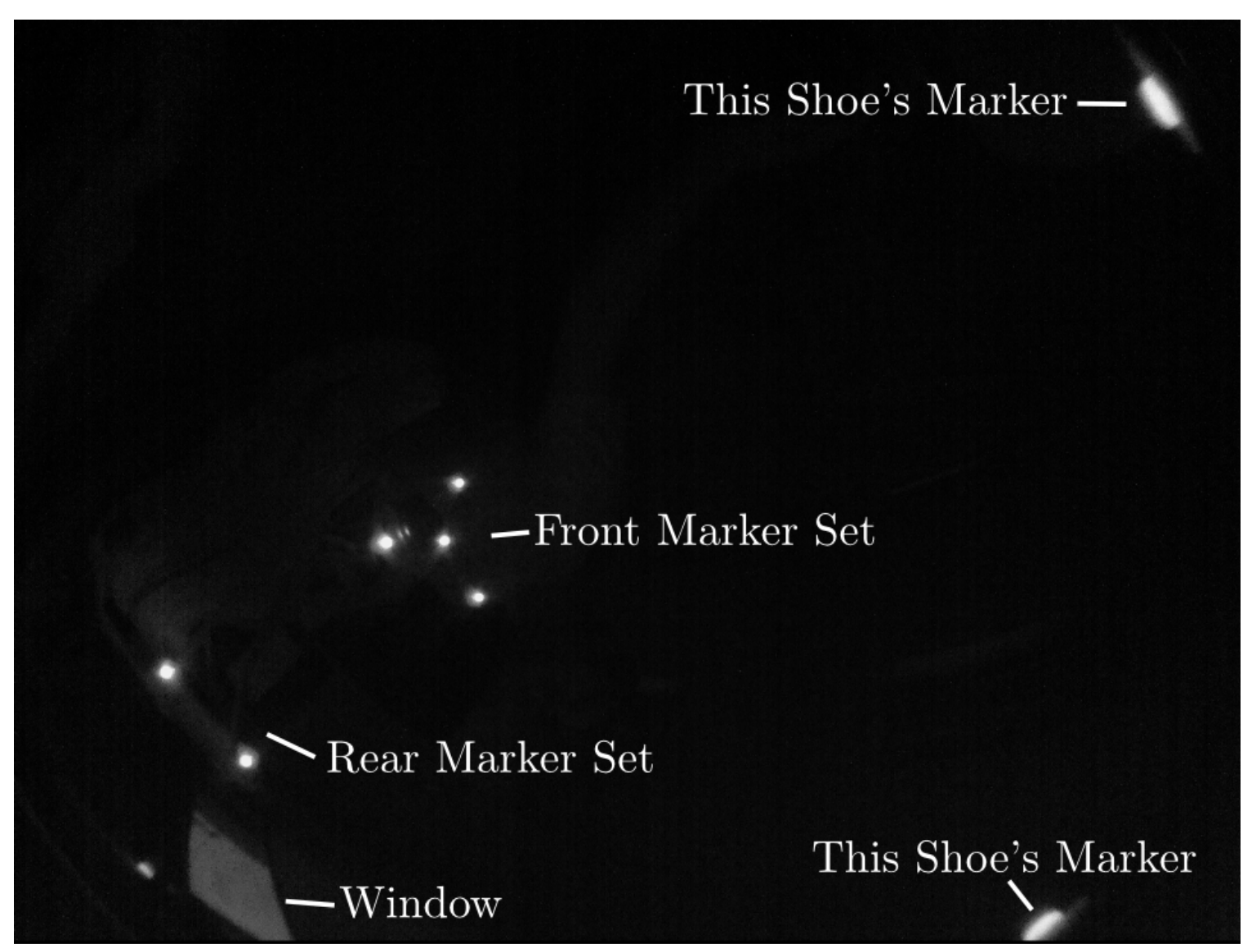

Figure 3.4.: Example of a captured image with labels. The bright dots are the infrared LED markers on both this shoe and the other shoe. The dim distorted rectangles are open windows on the room in which the image was captured.

derived from the blobs, such as the blob's size or the blob's direction.

The CCL is accomplished by scanning the image for white pixels, and then beginning a search starting from that pixel to find other white pixels connected to it. While searching, the algorithm records the location of all the connected white pixels it finds, and marks them so that they are not searched again later. When the search has found all of the connected white pixel of a particular blob, its centroid is calculated by averaging all of the stored pixel locations. The blob's size is the number of pixels which composed it. Once that blob is complete, the scanning process continues until new white pixels are found and searching begins again. This process continues until the entire image is scanned. 
Because of the limited processing power of the Raspberry Pi, it is not possible to check every pixel of the image during the scanning process. Instead, only every 6th column and row are scanned, resulting in a scanning process 36 times faster than checking every pixel. From experimentation, it was found that during regular operation, blobs remain much larger than 6 pixels across, and are rarely passed by. Additionally, when blobs are small enough to be passed by, the discretization error of a localization solution would be large enough that it is preferable to simply ignore the blob.

Once a white pixel is found, the connected white pixels are found using what is called a per-order depth-first-graph ${ }^{2}$ search (DFS). Besides its efficiency and common use, a DFS is used here because it allows for the sparse scanning just mentioned. Other CCL methods typically require every pixel to be checked.

Pseudocode for the connected component labelling and DFS are shown in Algorithm 3.1 .

\footnotetext{
${ }^{2}$ Here, the term graph is used in the mathematical sense and refers to a network of connected nodes. The image can be thought of as a network of nodes, where each pixel is a node and is connected to each of its 8 neighbours.
} 


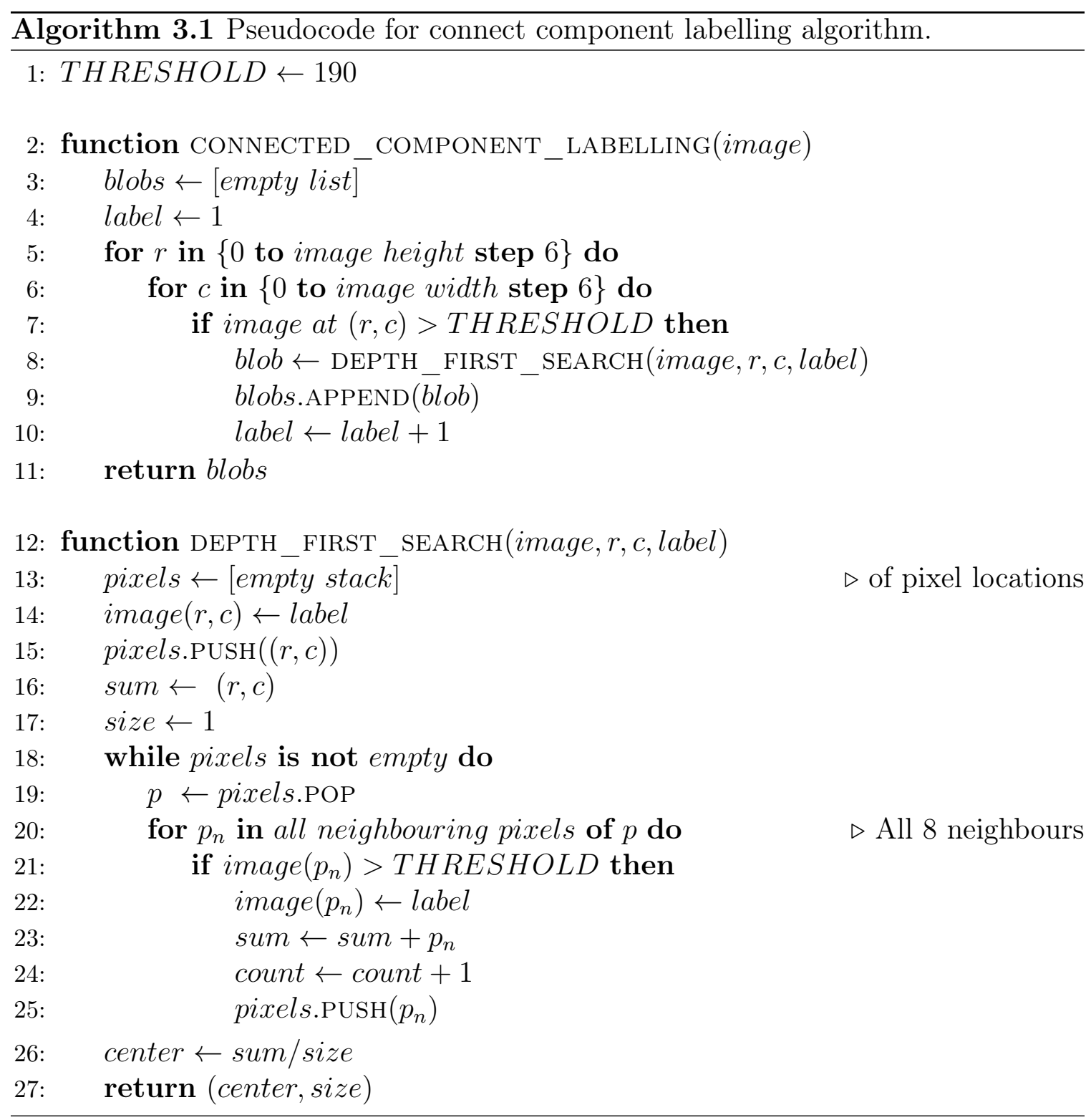

When complete, the connected component labelling algorithm produces a list of blobs, where each blobs has a location in the image in pixels and a size in square pixels. This can be visualized as shown in Figure 3.5, which is the same image as Figure 3.4, but with each blob separated from the others, as indicated by the different colours and numbers. 


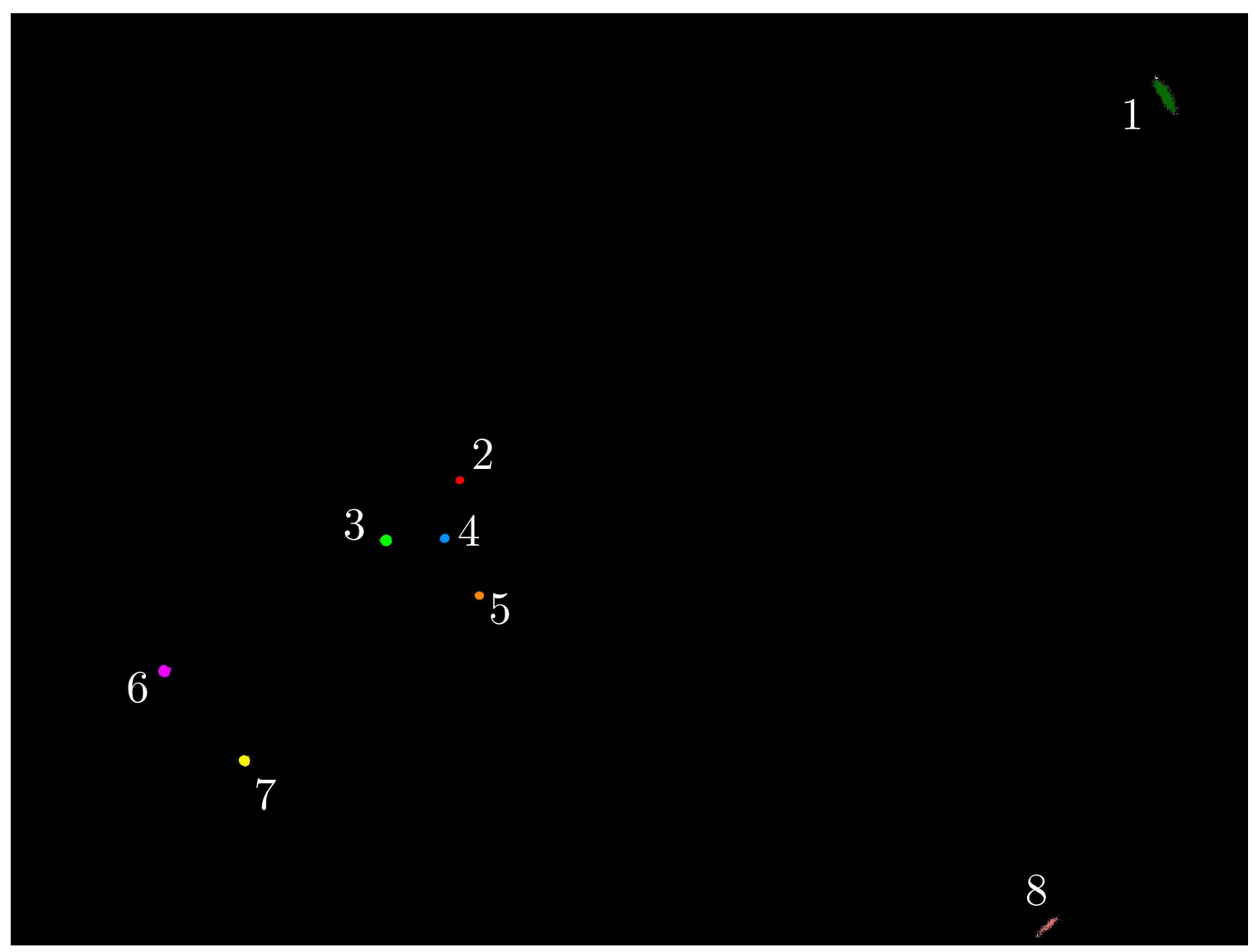

Figure 3.5.: Visualization of the output of connected component labelling. Each colour identifies all of the pixels belonging to a single blob.

\subsubsection{Blob Filtering}

In addition to the implicit filtering done by the thresholding in the previous section, a number of other steps are taken to ignore false blobs (blobs that do not correspond to a marker) before proceeding to finding the solution:

1. Any blob that has a size of less than 15 pixels is ignored. Typically when a connected component that small is found, it is not from a marker, but only a small island of brightness next to the true marker blob. Additionally, if a blob were to appear in the image with a size less than 15 pixels, it would be too far away or distorted to provide an accurate solution, and would best be discarded.

2. Each blob is checked against a list of known blob regions. If the marker is within 


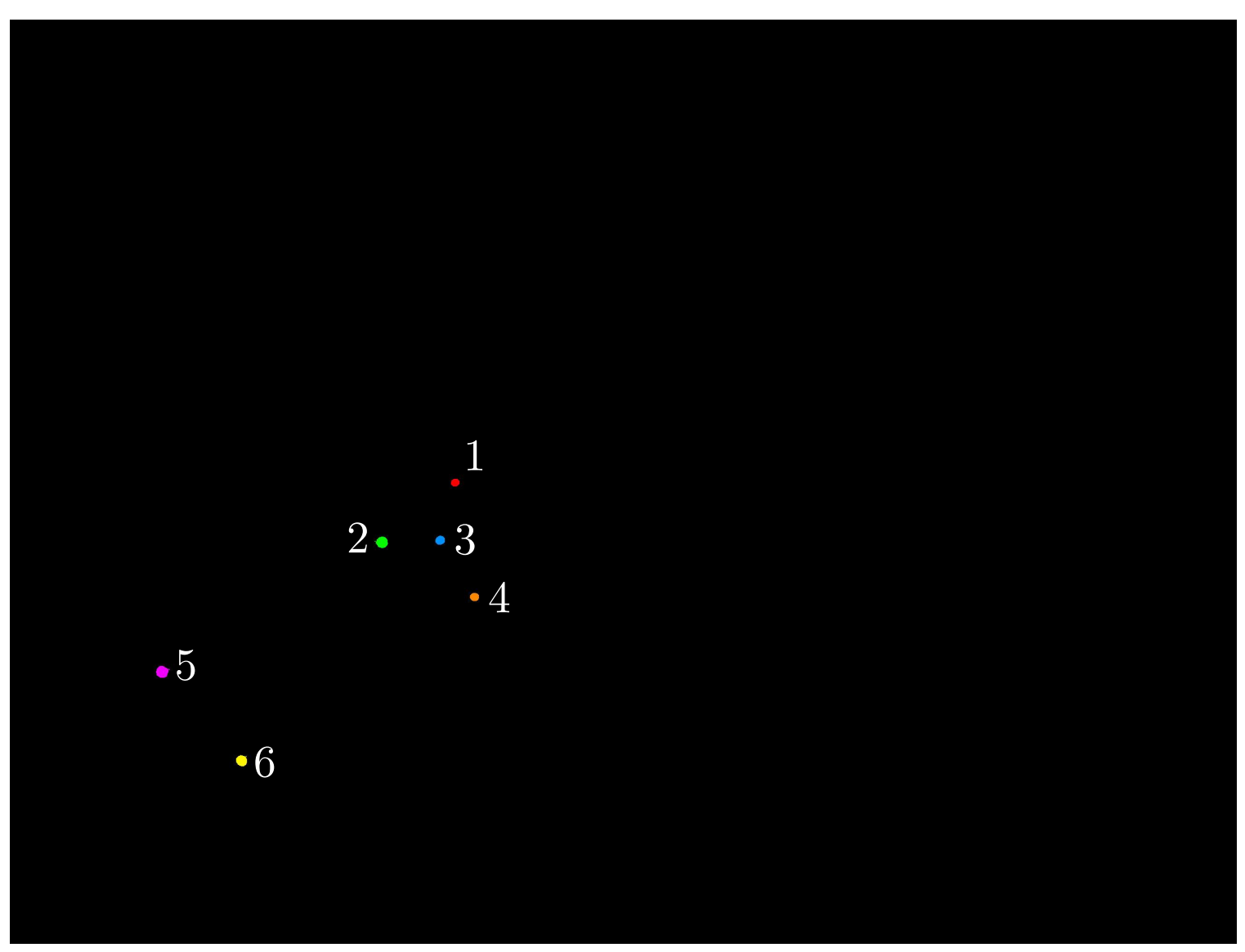

Figure 3.6.: Visualization of output of blob filtering.

one of these regions it is discarded. This step is necessary because some of the markers on the same shoe that captured the image will appear as blobs fixed to a certain location near the edge of the image, as shown in Figure 3.4.

3. If there are more than 8 blobs in the list, the list is sorted by size and the smallest blobs are removed until the list only contains 8 blobs. Since only 8 markers exist, more blobs than that indicates that the list contains false blobs. Typically, the false blobs are much smaller than the true blobs.

The result of this step is a list of blobs that all correspond to the markers on the other shoe. If the input image was Figure 3.4, the filtered blobs would be those shown in Figure 3.6. 


\subsubsection{The Find_Solution Algorithm}

The FIND_SOLUTION algorithm determines the position and orientation of the opposing shoe, $\boldsymbol{\Lambda}_{R L}$, from the list of blobs produced in the blob filtering step. Each blob in the list corresponds to a single marker on the other shoe, but which marker specifically is not yet known. Thus, the following algorithm must determine:

1. Which blob matches which marker;

2. If the list of blobs contains at least one entire marker set; and

3. Compute the localization solution, $\boldsymbol{\Lambda}_{R L}$, using that marker set.

To arrive at a single solution using the given information, the algorithm relies on a number of heuristic assumptions:

1. The markers' orientations with respect to the camera are limited by the range of motion of the human ankle. This makes it possible to determine which direction the front and top of the shoe are in. It also makes it possible to determine which blob matches which marker for a given marker set by knowing that certain markers will always be closer to the front of the shoe or closer to the top of the shoe than others.

2. Markers that are brighter will create larger blobs. In general, markers that are closer to the camera, or point closer ${ }^{3}$ to the camera's center will appear brighter and thus have larger blobs. Marker sets that are closer or whose LEDs point closer to the camera are often the best choice for calculating a solution. Thus blob size can be used to select the best marker set.

The FIND_SOLUTION algorithm will first be explained in pieces, with an explanation following each piece. The complete pseudocode will follow the explanations and can be found in Algorithm 3.2.

\footnotetext{
${ }^{3}$ The light that is emitted from the LED markers is not omnidirectional, but is brightest in one direction and dims when viewed at larger angles from the brightest direction.
} 


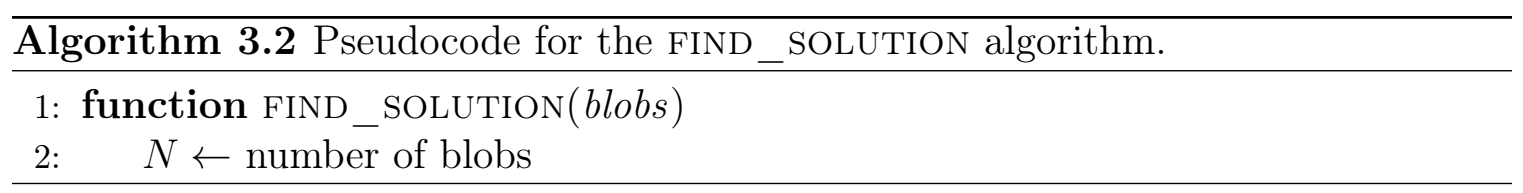

The algorithm has only a single input: the list of blobs produced in the blob filtering step. Each blob should correspond to a unique marker on the opposing shoe. $N$ is the the number of blobs passed to the function.

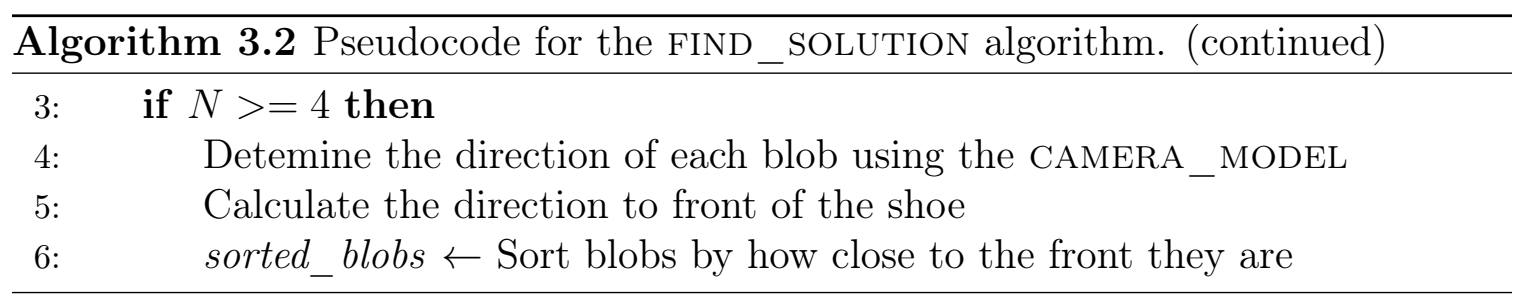

Since a complete marker set contains four markers, the algorithm only proceeds if four blobs were passed in input. The directions to the visible markers corresponding to the location of each blob in the image are calculated using the camera model. The camera model is described in detail in Section 3.2.4.

The direction to the front of the shoe is calculated by first calculating the average direction all of the directions calculated in the previous step. The direction to the front is simply the cross product of the average marker direction with the up direction. The up direction is a predefined set of coordinates unique to the shoe that is defined as normal to the sole of the shoe. Note that this is not the direction to the front of the shoe that captured the image, but of the shoe that is visible in the image. This calculation is not intended to provide a good estimate of the direction to the front of the shoe, but only provide a reference of the nominal direction to the front for the purpose of sorting the blobs. Finally, the blobs are sorted by the angle of their direction to the front direction. Blobs that have a smaller angle to the front direction are placed earlier in the list. 


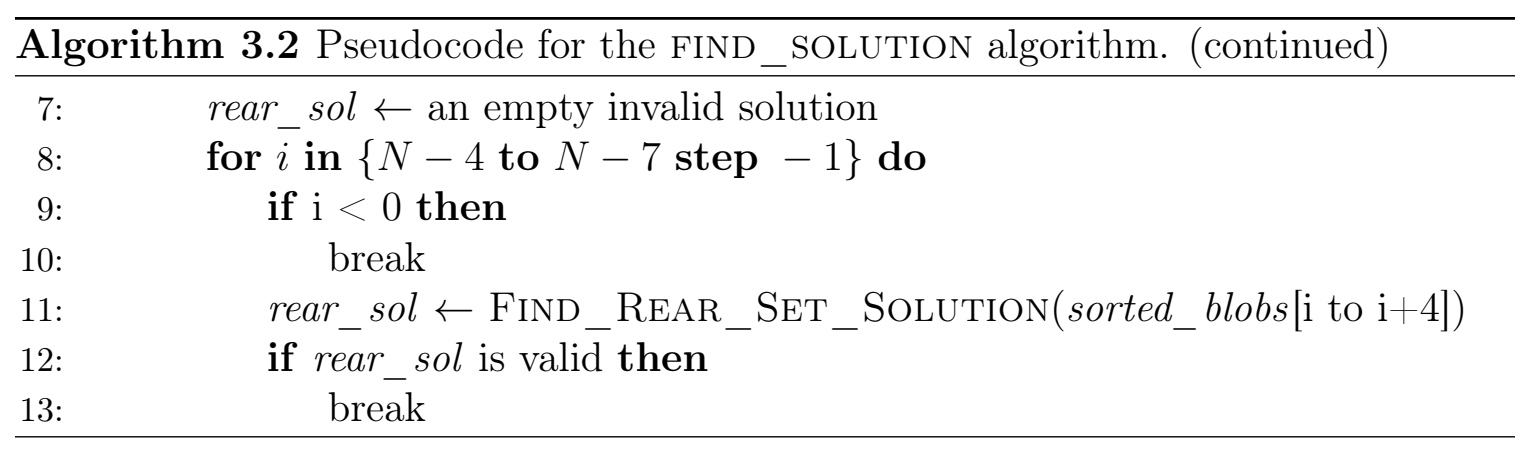

This portion of the algorithm searches the sorted blob list for valid solutions assuming the rear marker set is present. It begins by searching the rear-most blobs, then moves its search forward by one blob looking for a valid solution. It moves forward in this way three times. As soon as it finds a valid solution or runs out of blobs it stops searching, ending up with either a valid or invalid solution.

Ideally, checking the rear-most blobs for a valid rear-set solution would be sufficient as there are no markers farther to the rear than those in the rear-set. As mentioned in Section 1.2.6, fixed to the rear of each shoe is an Optotrak marker triplet. The Optotrak marker triplets produce infrared light that is visible to the cameras on the shoes. The marker triplets function by strobing on and off very quickly, and thus are not always visible in the image because of the camera low exposure time. In any given image, the Optotrak marker triplets add zero to two extra visible blobs at the rear of the shoe, and are indistinguishable from the markers used in the localization system. Thus, when searching for the rear-set solution, at most three possibilities must be checked. Fortunately, the geometry formed by the blobs when including the Optotrak markers is easily identifiable and is checked for in the FInD_REAR_SET_SOLUTION function.

The FIND_REAR_SET_SOLUTION function performs additional checks to validate the calculated solution and will be described in Section 3.2.5. 


\begin{tabular}{ll}
\hline Algorithm 3.2 & Pseudocode for the FIND_SOLUTION algorithm. (continued) \\
\hline 14: & front_blobs $\leftarrow$ sorted_blobs $[1$ to 4$]$ \\
15: & front_sol $\leftarrow$ FIND_FRONT_SET_SOLUTION $($ front_blobs $)$ \\
\hline
\end{tabular}

Compared to finding the rear-set solution, finding the front-set solution is much simpler: Either the front most blobs are a valid front-set solution or they are not. The FIND_FRONT_SET_SOLUTION function performs additional checks to validate the calculated solution and will be described in Section 3.2.5.

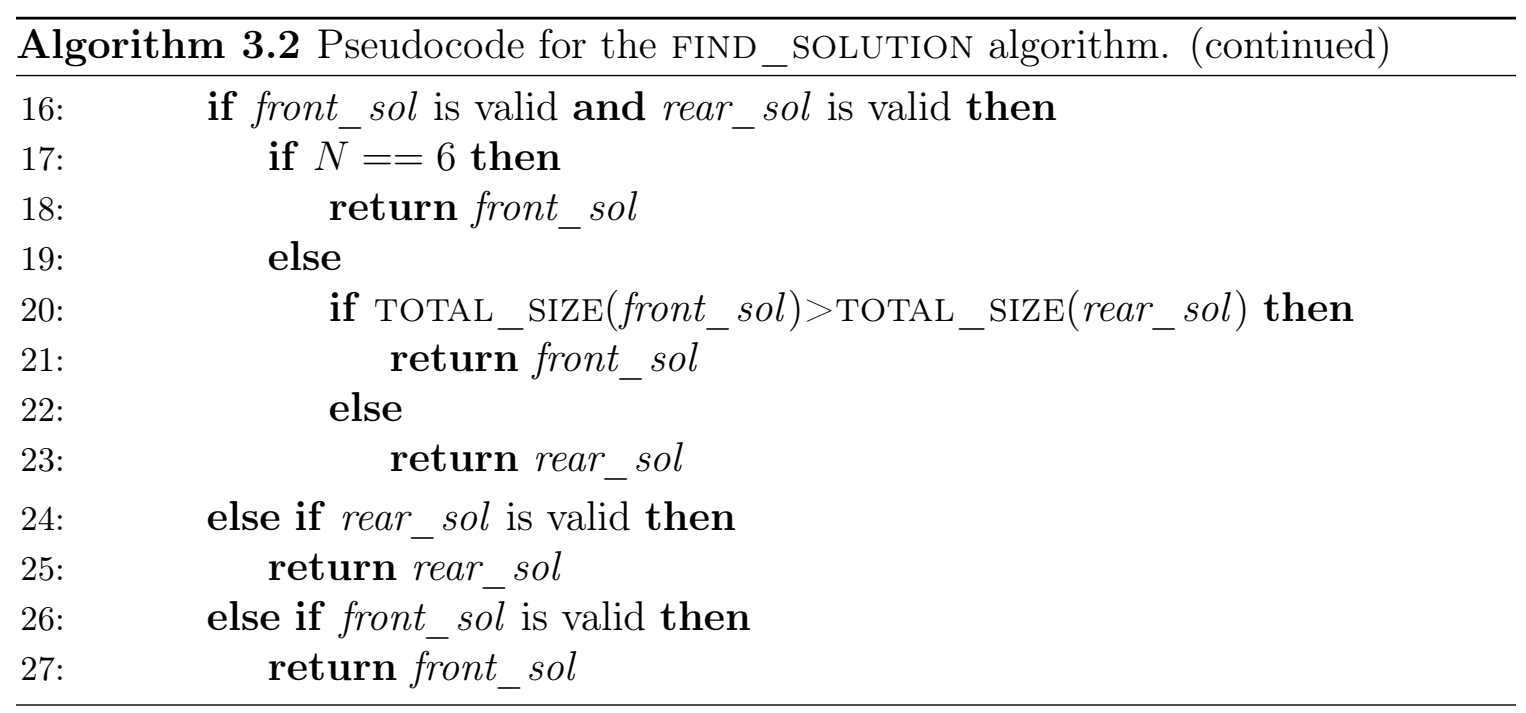

In this section of the code, three cases are handled:

1. If both the front-set solution and the rear-set solution are valid, then return the solution that was made from the larger blobs. There is one special case that was determined by experimentation: If there are exactly 6 markers visible, then the front-set solution should be returned. This is necessary because in this one situation the rear-set set solution has a chance of being incorrectly identified and has a larger total size than the front-set solution.

2. If only the rear-set solution is valid, then return the rear-set solution.

3. If only the front-set solution is valid, then return the front-set solution. 


\begin{tabular}{l}
\hline Algorithm 3.2 Pseudocode for the FIND_SOLUTION algorithm. (continued) \\
28: $\quad$ return an empty invalid solution
\end{tabular}

Finally, if a valid solution has not been found at this point, then return an invalid solution.

Below is the complete pseudocode for the FIND_SOLUTION algorithm.

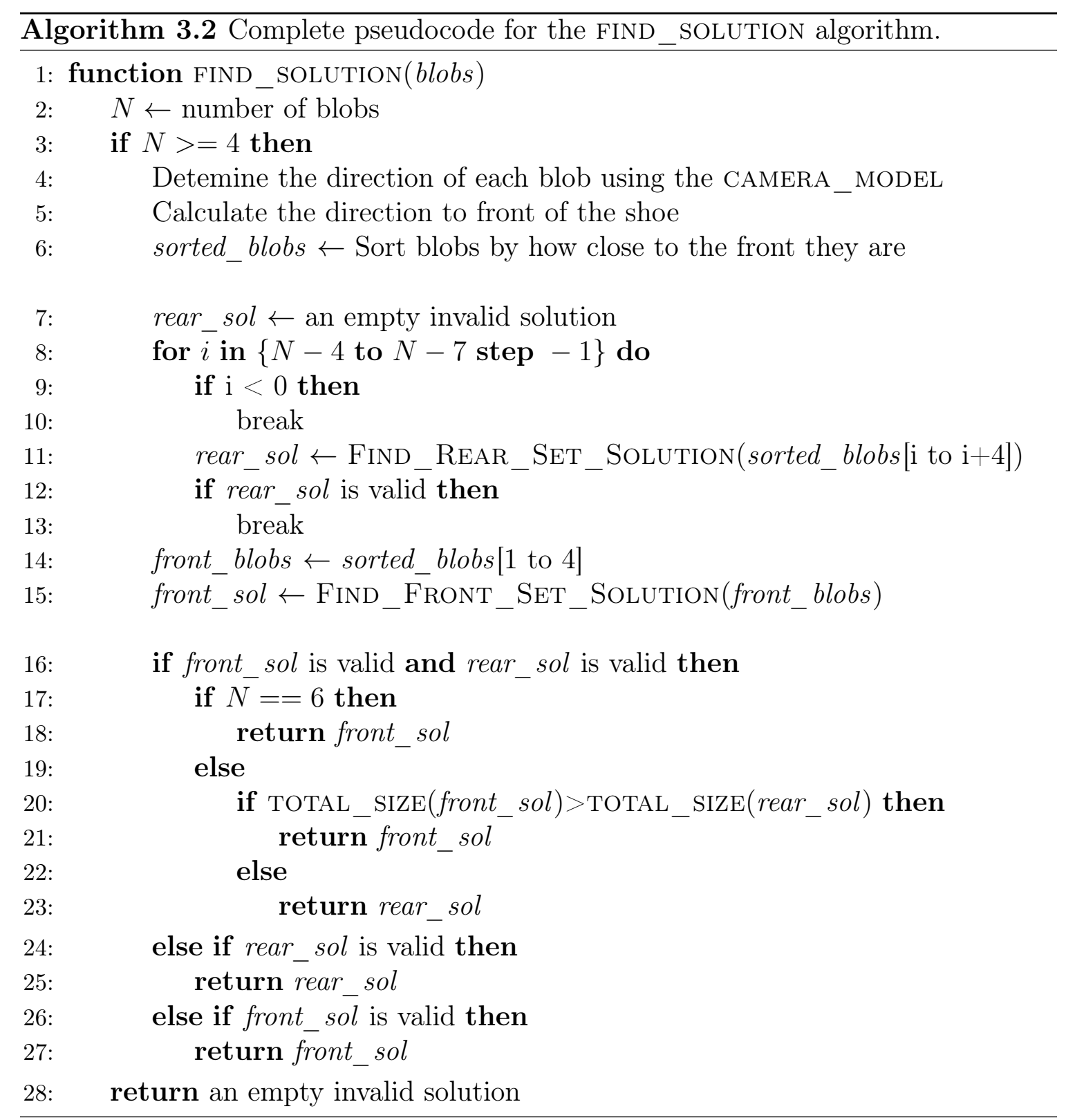




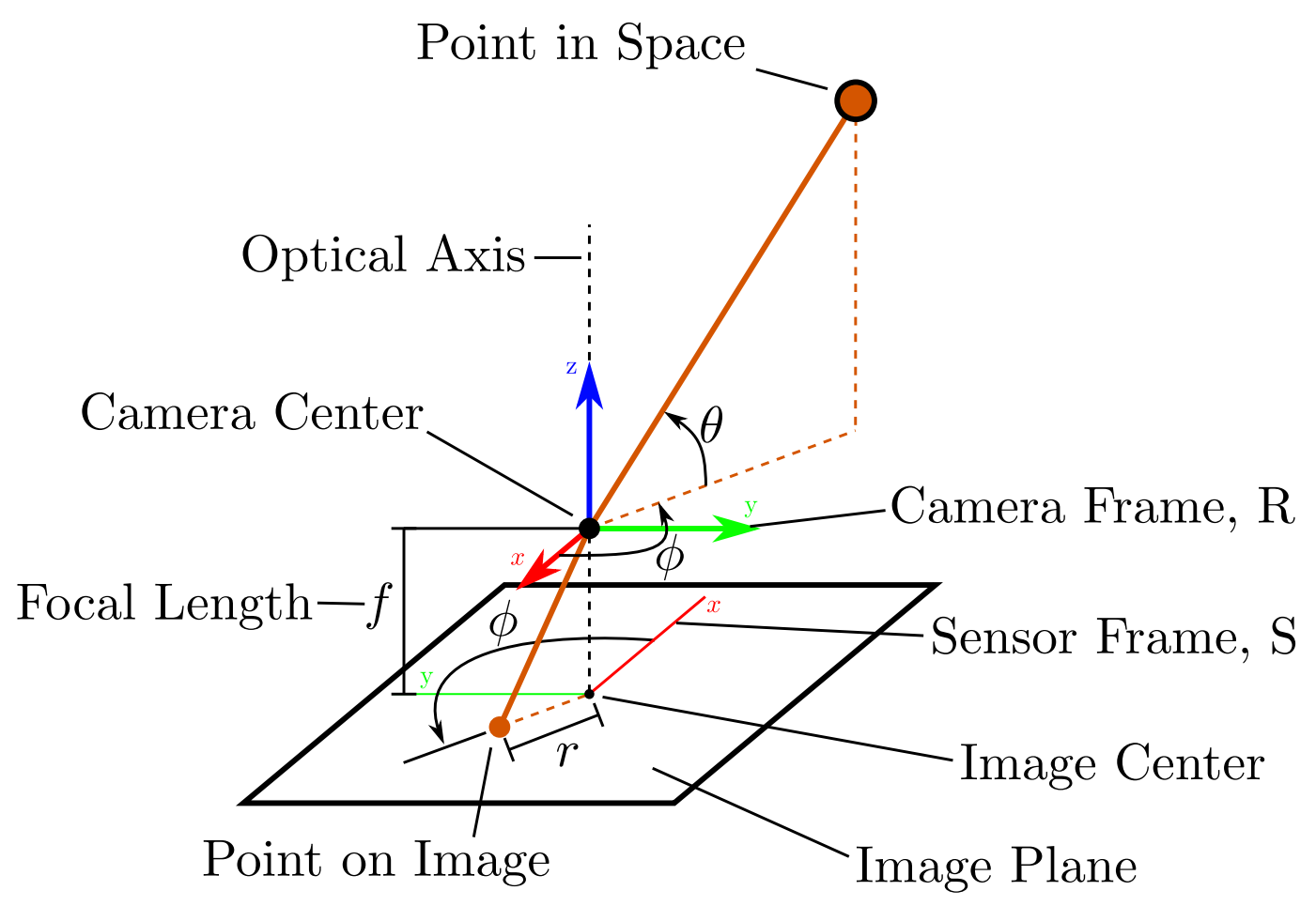

Figure 3.7.: Geometry of the camera model with labels.

\subsubsection{The Camera Model}

One of the key components of any vision system is the camera model. The camera model is a mathematical function or algorithm that relates the location of a point in front of the camera and the location of that point as it appears as in the image.

Figure 3.7 shows the geometry of a typical camera model. Many camera models employ a concept called the camera center (or lens center or optical center). In these models (called central camera models), the imaginary line which connects the point in space and the point in the image always passes through a point called the camera center (not necessarily in a straight line), as shown in Figure 3.7. The image center is the point where the optical axis intersects the image plane, where the optical axis is the direction the camera points in. The coordinate system placed at the camera center is called the camera frame. The camera frame's Z axis is aligned with the optical axis, and its $\mathrm{X}$ axis is aligned with the image's - $\mathrm{X}$ axis. The sensor frame 
is a coordinate system whose origin coincides with the image center and is aligned with the image frame. The image frame, shown below in Figure 3.8, has its origin in the top left corner of the image with its $\mathrm{X}$ axis pointing to the right, and its $\mathrm{Y}$ axis pointing down.

The direction to the point in space and the location of the point in the image can then be described using parameters relative to the camera frame and the sensor frame: $\theta$, $\phi$, and $r$. The camera model then relates these parameters algebraically.

Below, the term forward projection will refer to when a camera model is used to calculate the location of the point on the image, $(r, \phi)$, given the direction of a point in space, $(\theta, \phi)$. The opposite operation, backward projection, is when the direction in space $(\theta, \phi)$ is calculated given the location of a point in the image $(r, \phi)$. Many camera models work for both forward and backward projection, but some do not. To determine the localization solution, a model capable of efficiently computing the backward projection is necessary.

There are many camera models out there to choose from, such as the traditional pinhole or perspective model $(r=f \tan (\theta))$, or the equidistant fish-eye lens model $(r=f \theta)$. Unfortunately, these models are not complex enough to represent the camera used in this project. For example, they do not vary as a function of $\phi$, while the real camera does.

In [44], Kannala and Brandt describe a simple but very flexible camera model capable of very accurately describing fish-eye lenses and many of the accompanying distortions. Their model uses a radially symmetric polynomial mapping from $\theta$ to $r$, and then radial and tangential distortion terms that are products of a low order polynomials and Fourier series are applied. Unfortunately, their model was designed to be able to easily perform the forward projection; the backward projection can only be performed numerically. 
Since it is desirable to have a purely analytical backward projection, we propose to use a modification of Kannala and Brandt's model. Our model uses the same equations and distortion terms as their model, but the equations are applied in reverse order so that they do not need to be solved numerically. For example, Kannala and Brandt use:

$$
r=D_{1} \theta+D_{2} \theta^{3}+D_{3} \theta^{5}+D_{4} \theta^{7}+D_{5} \theta^{9},
$$

while our model uses:

$$
\theta=D_{1} r+D_{2} r^{3}+D_{3} r^{5}+D_{4} r^{7}+D_{5} r^{9}
$$

The algorithm, which is described below, has:

1. two inputs: $p$ and $q$, the coordinates of a point in the image in pixels; and

2. two outputs: $\theta$ and $\phi$, the direction in the camera frame in spherical coordinates.

The variables listed in Table 3.1 are tunable parameters used in the camera model that are determined though a calibration process that will be described in Section 4.3 .

To help illustrate the intermediate parameters calculated below, Figure 3.8 depicts them geometrically.

Variables $p$ and $q$ are the Cartesian coordinates of point $D$ in the image frame with units of pixels. The first step is to calculate the coordinates of point $D$ in the sensor frame with units of millimeters using 


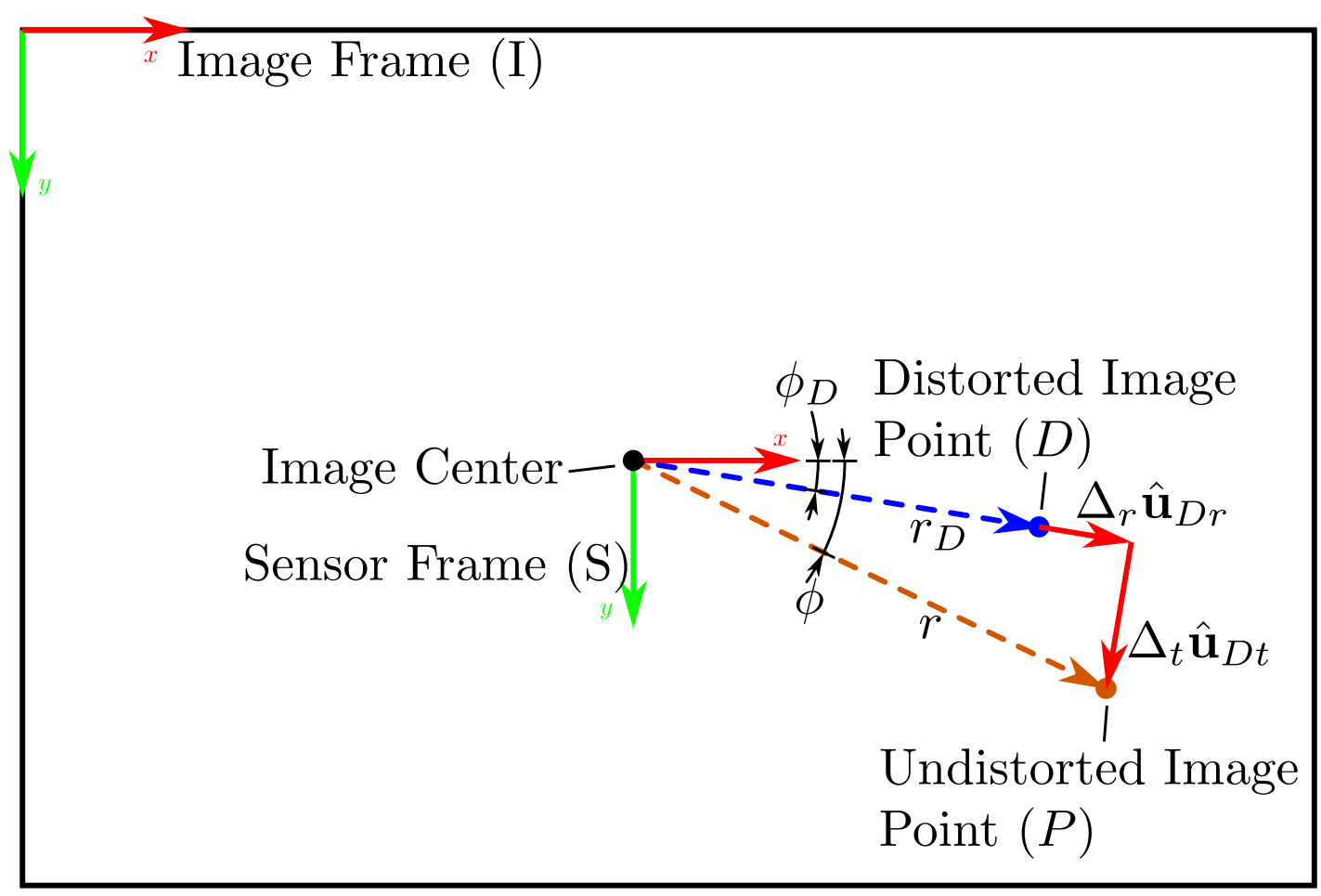

Figure 3.8.: Depiction of parameters used in the camera model. The $r$ and the $t$ subscripts indicate the radial and tangential directions.

$$
\begin{aligned}
& r_{D}=\left\|\mathbf{v}_{S D, S}\right\| \\
& \phi_{D}=\tan ^{-1}\left(\frac{v_{D y}}{v_{D x}}\right) .
\end{aligned}
$$

Note that when $\tan ^{-1}$ is used here and below, special attention should be paid to calculating the correct quadrant. In most programming languages, this is done with the ARCTAN2() or ATAN2() function.

Now the distortion terms $\Delta_{r}$ and $\Delta_{t}$ can be calculated using the equations from [44]: 


$$
\begin{aligned}
\Delta_{r} & =\left(L_{1} r_{D}+L_{2} r_{D}^{3}+L_{3} r_{D}^{5}\right)\left(I_{1} \cos \phi_{D}+I_{2} \sin \phi_{D}+I_{3} \cos 2 \phi_{D}+I_{4} \sin 2 \phi_{D}\right) \\
\Delta_{t} & =\left(M_{1} r_{D}+M_{2} r_{D}^{3}+M_{3} r_{D}^{5}\right)\left(J_{1} \cos \phi_{D}+J_{2} \sin \phi_{D}+J_{3} \cos 2 \phi_{D}+J_{4} \sin 2 \phi_{D}\right) .
\end{aligned}
$$

Now we can apply the distortion terms to the distorted image point to calculate the undistorted image point $P$ using

$$
\mathbf{v}_{S P, S}=\left(\begin{array}{c}
v_{P x} \\
v_{P y}
\end{array}\right)=\left(r_{D}+\Delta_{r}\right) \hat{\mathbf{u}}_{D r}+\Delta_{t} \hat{\mathbf{u}}_{D t} .
$$

where

$$
\begin{aligned}
& \hat{\mathbf{u}}_{D r}=\left(\begin{array}{c}
\cos \phi_{D} \\
\sin \phi_{D}
\end{array}\right) \\
& \hat{\mathbf{u}}_{D t}=\left(\begin{array}{c}
-\sin \phi_{D} \\
\cos \phi_{D}
\end{array}\right)
\end{aligned}
$$

Next, we can convert the undistorted image point $\mathbf{v}_{S P, S}$ to polar coordinates $r$ and $\phi$ with

$$
\begin{aligned}
r & =\left\|\mathbf{v}_{S P, S}\right\| \\
\phi & =\tan ^{-1}\left(\frac{v_{P y}}{v_{P x}}\right) .
\end{aligned}
$$

Finally, using the radius of the undistorted image point, $\theta$ can be calculated using a 
radially symmetric polynomial of the same form as the mapping from [44]:

$$
\theta=D_{1} r+D_{2} r^{3}+D_{3} r^{5}+D_{4} r^{7}+D_{5} r^{9} .
$$

The coordinate pair $(\theta, \phi)$ is the desired direction in camera frame in spherical coordinates as shown in Figure 3.7. In the software, the direction is converted to a unit vector, $\hat{\mathbf{v}}_{R n, R}$ for marker $n$, which is required for the FIND_FRONT/REAR_SET_SOLUTION algorithms.

Just as Kannala and Brandt's model is efficient at calculating the forward projection, but not the backward projection, this model is efficient at calculating the backward projection, but not the forward projection. Fortunately, during real-time operation, calculating the forward projection is never necessary. The only case when it is necessary to do forward projection is during the camera calibration procedure, which will be described in Section 4.3. In the forward projection case, the image point is calculated using a numerical root finding algorithm and the model described above. An initial estimate is made using the above model, but neglecting all of the distortion terms except $D_{1}, S_{1}, S_{2}, p_{0}$, and $q_{0}$. The resulting simplification is easily invertible, and provides an acceptable estimate.

The simplification of the model is:

$$
\mathbf{v}_{S P, S}=\left(\begin{array}{l}
v_{x} \\
v_{y}
\end{array}\right)=\left(\begin{array}{cc}
S_{1} & 0 \\
0 & S_{2}
\end{array}\right)\left(\left(\begin{array}{l}
p \\
q
\end{array}\right)-\left(\begin{array}{l}
p_{0} \\
q_{0}
\end{array}\right)\right),
$$




$$
\begin{aligned}
& r=\left\|\mathbf{v}_{S P, S}\right\| \\
& \phi=\tan ^{-1}\left(\frac{v_{y}}{v_{x}}\right),
\end{aligned}
$$

$$
\theta=D_{1} r
$$

The inverted simplified model used to make an initial estimate is:

$$
\begin{gathered}
r=\frac{\theta}{D_{1}} \\
\mathbf{v}_{S P, S}=r\left(\begin{array}{c}
\cos \phi \\
\sin \phi
\end{array}\right) \\
\left(\begin{array}{l}
p \\
q
\end{array}\right)=\left(\begin{array}{cc}
\frac{1}{S_{1}} & 0 \\
0 & \frac{1}{S_{2}}
\end{array}\right) \mathbf{v}_{S P, S}+\mathbf{v}_{I S, I}
\end{gathered}
$$

\subsubsection{The Find_Front/Rear_Set_Solution Algorithms}

At this point in the FIND_SOLUTION algorithm, four of the blobs have been identified for calculating a solution. Four steps are followed to calculate a solution given these four blobs:

1. Check whether the four blobs are arranged in the correct configuration;

2. For the given marker set, determine to which marker each blob corresponds;

3. Calculate the solution; 
Front of the Shoe
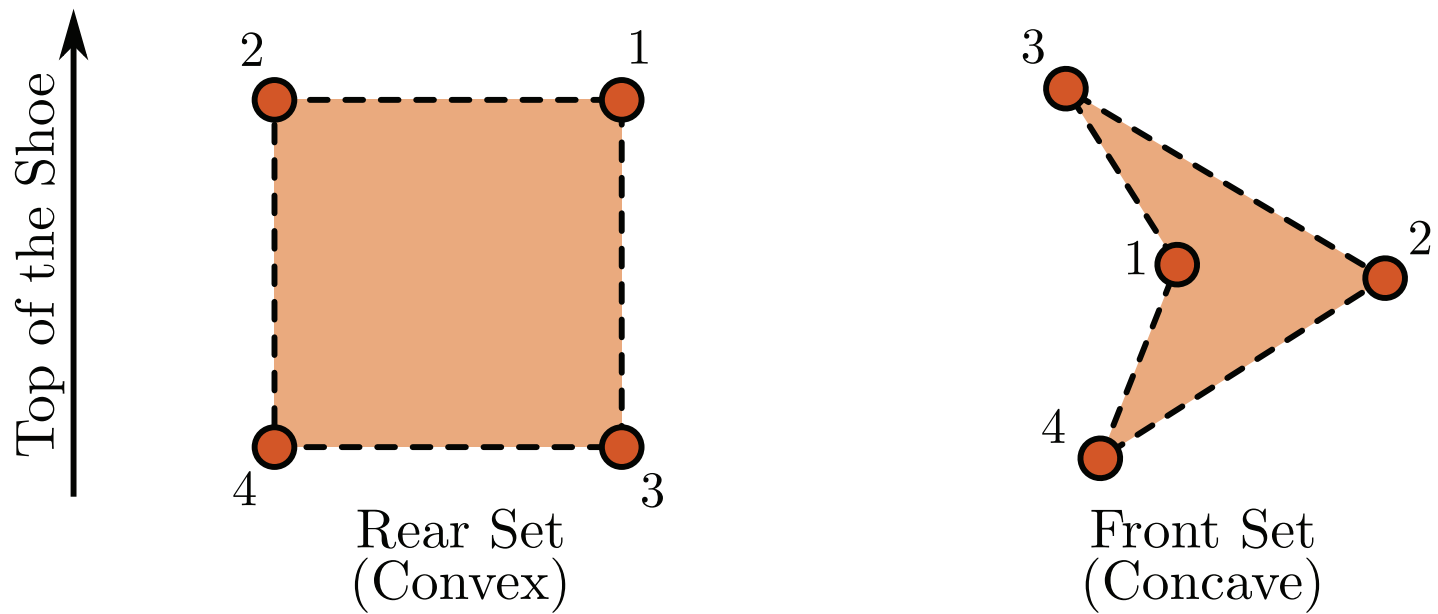

Figure 3.9.: Marker arrangement in the two marker sets.

4. Check whether the solution is valid.

To help differentiate the two sets of markers during processing, the markers of each set are arranged in two unique configurations, as shown in Figure 3.9.

If the markers are connected with imaginary lines as shown in Figure 3.9, they form two distinct types of quadrilaterals. The front set forms what is called a concave quadrilateral, while the rear set forms a convex quadrilateral. These two types of quadrilaterals are very useful because regardless of what angle they are viewed at, they remain convex or concave. Thus, given four blobs from either the front set or rear set, it is possible to determine which set they belong to by checking whether they form a concave or convex quadrilateral.

To determine whether a set of points forms a concave quadrilateral, each point is checked to see if it lies inside the triangle formed by the other three points. If a single point lies in the triangle formed by the other points, then those four points form a concave quadrilateral. To test whether a given point lies within a triangle the barycenter coordinate method is used. 
Once it is known to which set the given blobs belong, it is necessary to know which blob in our given list corresponds to which marker in the specified set. This is achieved by sorting the given list of blobs in a number of ways until they end up in a known order. The expected order of markers for the two sets is indicated in Figure 3.9 by the numbers next to each marker.

For the front set, the sorting procedure is as follows:

1. Calculate the direction to the center of the four blobs, by averaging the four spatial directions.

2. Sort the four blobs by how close they are to the center direction, so that the closest blob is now the first in the list.

3. Sort the last three blobs by how close they are to the front of the shoe.

4. Sort the last two blobs by how close they are to the top of the shoe.

For the rear set, the sorting procedure is:

1. Sort the entire list by how close the blobs are to the top of the shoe.

2. Sort the first two blobs by how close they are to the front of the shoe.

3. Sort the last two blobs by how close they are to the front of the shoe.

After sorting, it is easy to get a specific blob by looking at a certain element in the list.

Once it is known which marker matches each blob, it is finally possible to calculate $\Lambda_{R L}$. The first step is to calculate the distance to each marker.

The problem of determining the distance to $n$ points given only their directions and their position relative to each other is referred to as the perspective $n$ point $(\mathrm{PnP})$ problem, and has many known solutions. In this case, $n=4$. In [45], Fabrizio and Devars have provided a very elegant solution to the P4P problem for 4 coplanar points, and this solution has been adopted for this system. 


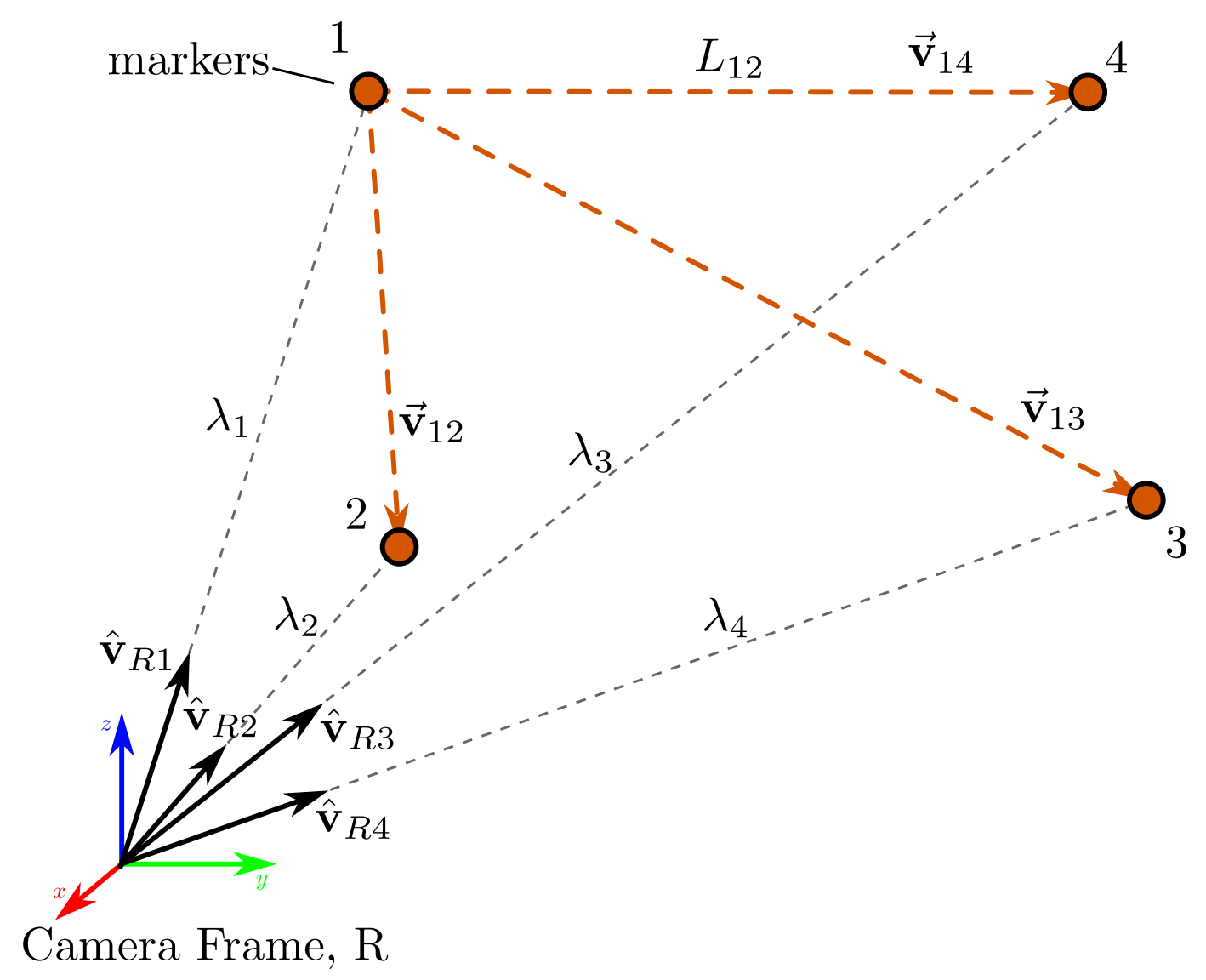

Figure 3.10.: Depiction of values used in Fabrizio and Devars' solution to the P4P problem using coplanar points.

Figure 3.10 shows the geometry and parameters used in Fabrizio and Devars' solution. Once again, the $\mathrm{P} 4 \mathrm{P}$ problem here is: given the unit vectors $\hat{\mathbf{v}}_{R 1}, \hat{\mathbf{v}}_{R 2}, \hat{\mathbf{v}}_{R 3}$, and $\hat{\mathbf{v}}_{R 4}$ and the vectors $\overrightarrow{\mathbf{v}}_{12}, \overrightarrow{\mathbf{v}}_{13}$, and $\overrightarrow{\mathbf{v}}_{14}$, what are the distances to the markers, $\lambda_{1}, \lambda_{2}, \lambda_{3}$, and $\lambda_{4}$. Instead of using $\overrightarrow{\mathbf{v}}_{12}, \overrightarrow{\mathbf{v}}_{13}$, and $\overrightarrow{\mathbf{v}}_{14}$ directly, Fabrizio and Devars make use of the scalar parameters:

- $L_{12}$ is the distance from marker 1 to marker 2 , and

- parameters $K_{1}$ and $K_{2}$ (not shown in the diagram) must satisfy the following equation:

$$
\overrightarrow{\mathbf{v}}_{13}=K_{1} \overrightarrow{\mathbf{v}}_{12}+K_{2} \overrightarrow{\mathbf{v}}_{14}
$$


That is, $K_{1}$ and $K_{2}$ are the coordinates of $\overrightarrow{\mathbf{v}}_{13}$ using $\overrightarrow{\mathbf{v}}_{12}$ and $\overrightarrow{\mathbf{v}}_{14}$ as a vector basis. This is only strictly possible if all four markers are coplanar and $\overrightarrow{\mathbf{v}}_{12}$ is not parallel to $\overrightarrow{\mathbf{v}}_{14}$. The values of $L_{12}, K_{1}$, and $K_{2}$ are determined using the calibration procedure that will be described in Section 4.5.

The distances to the markers, $\lambda_{1}, \lambda_{2}, \lambda_{3}$, and $\lambda_{4}$ can then be calculated with [45]:

$$
\begin{aligned}
\lambda_{1} & =\frac{L_{12}}{\left\|\zeta \hat{\mathbf{v}}_{R 2, R}-\hat{\mathbf{v}}_{R 1, R}\right\|}, \\
\lambda_{2} & =\zeta \lambda_{1}, \\
\lambda_{3} & =\frac{-K_{1} \Delta_{214}}{\Delta_{134}} \zeta \lambda_{1}, \\
\lambda_{4} & =\frac{K_{1} \Delta_{231}}{K_{2} \Delta_{134}} \zeta \lambda_{1},
\end{aligned}
$$

where

$$
\zeta=\frac{\left(K_{1}+K_{2}-1\right) \Delta_{134}}{K_{1} \Delta_{234}}
$$

and

$$
\Delta_{i j k}=\left|\begin{array}{lll}
\hat{\mathbf{v}}_{R i, R} & \hat{\mathbf{v}}_{R j, R} & \hat{\mathbf{v}}_{R k, R}
\end{array}\right| .
$$

The $3 \times 1$ column matrix $\hat{\mathbf{v}}_{R n, R}$ contains the Cartesian coordinates of $\hat{\mathbf{v}}_{R n}$ in camera frame $R$, and $\Delta_{i j k}$ denotes the determinant of the $3 \times 3$ matrix formed by horizontally stacking the column vectors $\hat{\mathbf{v}}_{R i, R}, \hat{\mathbf{v}}_{R j, R}$, and $\hat{\mathbf{v}}_{R k, R}$.

The second step in calculating the localization solution, $\boldsymbol{\Lambda}_{R L}$, is to determine the rotation and translation from the left shoe to the right shoe. Consider two separate coordinate systems, $A$ and $B$, as shown in Figure 3.11, with some difference in their 


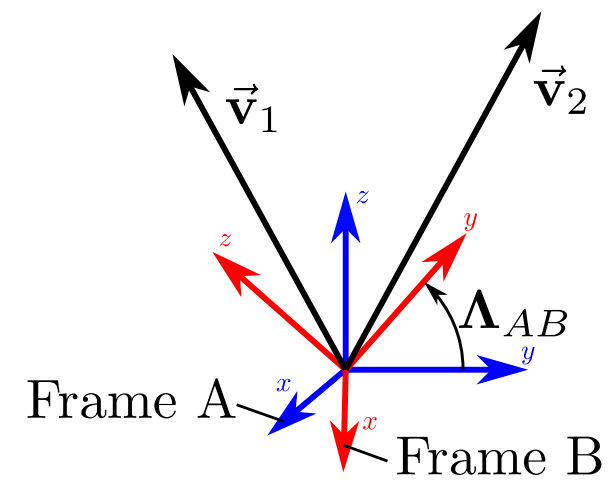

Figure 3.11.: Geometry of a simple attitude estimation problem.

relative orientation. Consider also, two unit vectors, $\hat{\mathbf{v}}_{1}$ and $\hat{\mathbf{v}}_{2}$, representing two different directions. Because of their rotational offset, each frame will have different sets of coordinates for those two directions: $\left(\mathbf{v}_{1, A}, \mathbf{v}_{1, B}\right)$ and $\left(\mathbf{v}_{2, A}, \mathbf{v}_{2, B}\right)$. The problem at hand is to find $\boldsymbol{\Lambda}_{A B}$, knowing only $\mathbf{v}_{1, A}, \mathbf{v}_{1, B}, \mathbf{v}_{2, A}$, and $\mathbf{v}_{2, B}$. This problem is well known in spacecraft dynamics as attitude estimation, where attitude is a synonym for orientation.

The calculation of the relative orientation between the shoes is precisely the same problem with the additional complication that two coordinate systems do not share the same origin, but this can be easily overcome. Figure 3.12 shows a simplified version of the attitude estimation problem for the shoes.

Each shoe has its own fixed coordinate system (each shoe's camera frame), $R$ on this foot and $L$ on the other. At the current point in the localization algorithm, the position of the markers is known in both coordinate systems. In Camera Frame $R$, it was calculated using the captured images as discussed above. In $L$, the positions of the markers are fixed and are measured in advance, as will be discussed in Section 4.5. Because of the translation between $R$ and $L$, the position of marker $n$ with respect to frame $R$ is not the same vector as the position of the same marker with respect to frame $L$, as is clear in Figure 3.12. Instead, we use the position of the markers relative to each other, vectors $\overrightarrow{\mathbf{v}}_{12}, \overrightarrow{\mathbf{v}}_{13}$, and $\overrightarrow{\mathbf{v}}_{14}$. The coordinates of these vectors can 


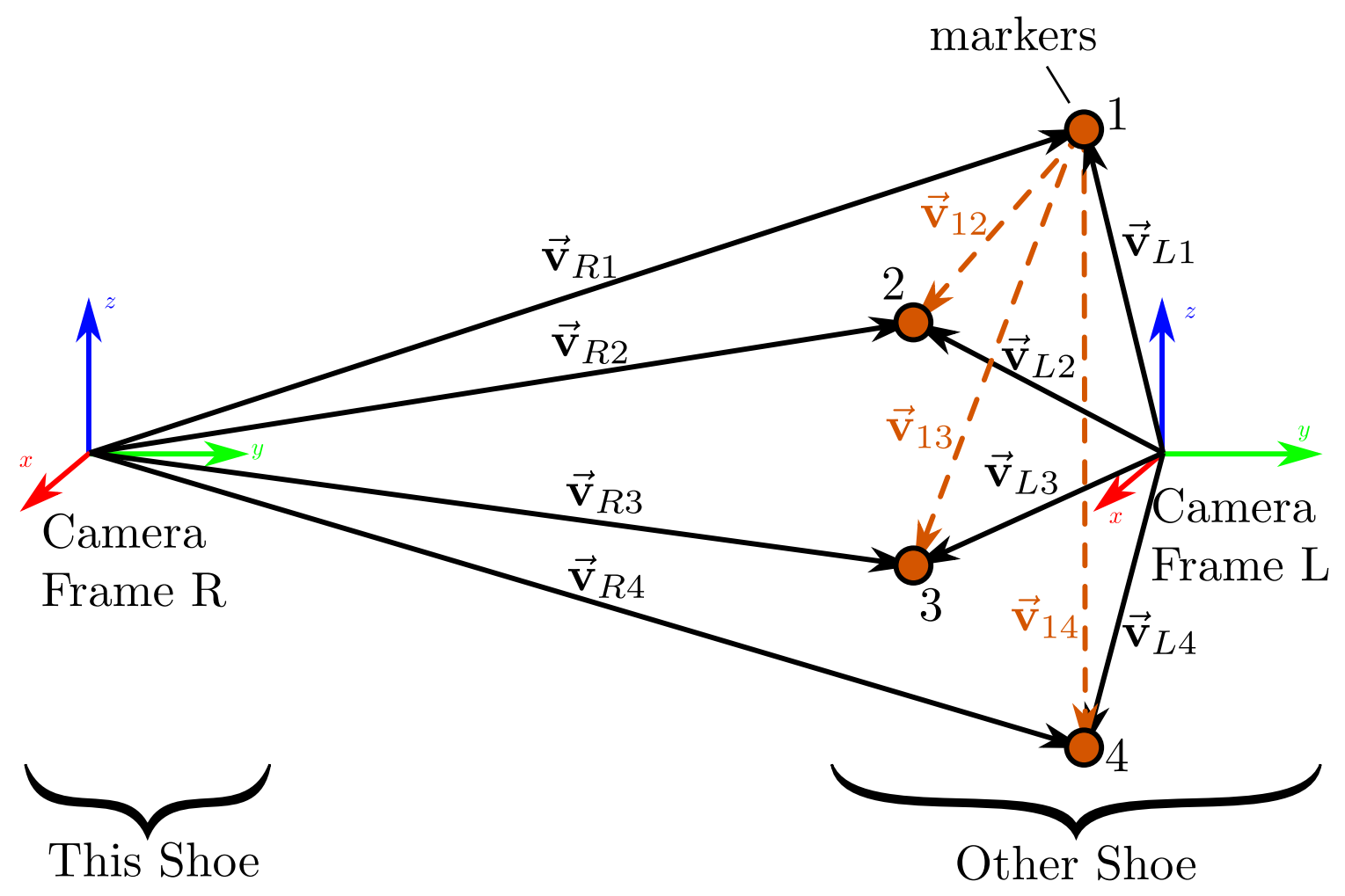

Figure 3.12.: Geometry of coordinate systems and markers for the shoe localization problem.

be easily calculated in both reference frames.

In practice, it is often impossible to find a rotation that is able to transform all the coordinates perfectly because of the inherent noise in the coordinates that derive from sensor measurements. The problem usually becomes one of finding the rotation that minimizes the error of coordinate transformation. While minimization often requires a numerical solution, this particular minimization problem actually has an analytical solution.

While a number of solutions exist, Davenport's Q-Method[46] is implemented here for its simplicity and robustness. First, we calculate the coordinates of the three vectors $\overrightarrow{\mathbf{v}}_{12}, \overrightarrow{\mathbf{v}}_{13}$, and $\overrightarrow{\mathbf{v}}_{14}$ in frames $R$ and $L$ : 


$$
\begin{aligned}
& \mathbf{v}_{12, R}=\lambda_{2} \hat{\mathbf{v}}_{R 2, R}-\lambda_{1} \hat{\mathbf{v}}_{R 1, R} \\
& \mathbf{v}_{13, R}=\lambda_{3} \hat{\mathbf{v}}_{R 3, R}-\lambda_{1} \hat{\mathbf{v}}_{R 1, R} \\
& \mathbf{v}_{14, R}=\lambda_{4} \hat{\mathbf{v}}_{R 4, R}-\lambda_{1} \hat{\mathbf{v}}_{R 1, R} \\
& \mathbf{v}_{12, L}=\mathbf{v}_{L 2, L}-\mathbf{v}_{L 1, L} \\
& \mathbf{v}_{13, L}=\mathbf{v}_{L 3, L}-\mathbf{v}_{L 1, L} \\
& \mathbf{v}_{14, L}=\mathbf{v}_{L 4, L}-\mathbf{v}_{L 1, L}
\end{aligned}
$$

Next, we can compute $K$, a $4 \times 4$ matrix sometimes referred to as the Davenport Matrix:

$$
\mathbf{K}=\left(\begin{array}{cc}
\mathbf{S}-\sigma \mathbf{I}_{3} & \mathbf{Z} \\
\mathbf{Z}^{T} & \sigma
\end{array}\right)
$$

where

$$
\begin{gathered}
\mathbf{I}_{3}=\left(\begin{array}{ccc}
1 & 0 & 0 \\
0 & 1 & 0 \\
0 & 0 & 1
\end{array}\right) \\
\mathbf{B}=\mathbf{v}_{12, R} \mathbf{v}_{12, L}^{T}+\mathbf{v}_{13, R} \mathbf{v}_{13, L}^{T}+\mathbf{v}_{14, R} \mathbf{v}_{14, L}^{T} \\
\mathbf{S}=\mathbf{B}+\mathbf{B}^{T}, \\
\sigma=\operatorname{trace}(\mathbf{B}),
\end{gathered}
$$


and

$$
\mathbf{Z}=\left(\begin{array}{l}
\mathbf{B}_{23}-\mathbf{B}_{32} \\
\mathbf{B}_{31}-\mathbf{B}_{13} \\
\mathbf{B}_{12}-\mathbf{B}_{21}
\end{array}\right)
$$

Next, we compute the eigenvalues and eigenvectors of matrix $K$. The eigenvector that corresponds to the largest eigenvalue will be the optimal ${ }^{6}$ coordinate system transformation, in the form of a unit quaternion. That is, if the eigenvector is $\left(\begin{array}{llll}a_{1} & a_{2} & a_{3} & a_{4}\end{array}\right)^{T}$, then the quaternion transformation is $\mathbf{q}_{R L}=a_{4}+a_{1} i+a_{2} j+a_{3} k$. Lastly, the location of frame $L$ in frame $R, \mathbf{v}_{R L, R}$, can be calculated using any marker $i$ with:

$$
\mathbf{v}_{R L, R}=\mathbf{v}_{L i, L}-\mathbf{q}_{R L}^{*} \mathbf{v}_{R i, R} \mathbf{q}_{R L}
$$

The translation and rotation, $\mathbf{v}_{R L, R}$ and $\mathbf{q}_{R L}$, represent the coordinate system transformation from one shoe's camera frame to the other, and is the final result of the shoevision process:

$$
\boldsymbol{\Lambda}_{R L}=\left(\mathbf{v}_{R L, R}, \mathbf{q}_{R L}\right)
$$

After calculation, $\boldsymbol{\Lambda}_{R L}$ is sent to the integrator process to be combined with the measurement from the other shoe, filtered and logged. 


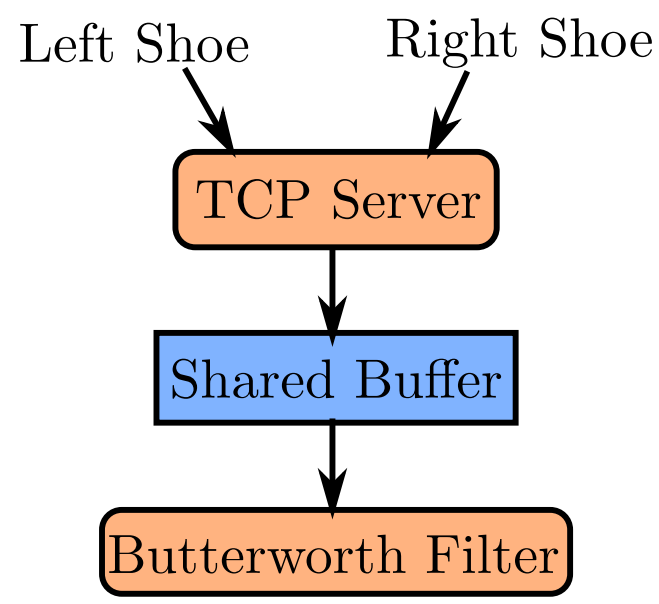

Figure 3.13.: Overview of the integrator process.

\subsection{Integrator}

The integrator process is the second phase of the data processing pipeline. It receives the outputs of the two shoevision processes, combines them together, applies a smoothing filter, and outputs the final estimate of the transformation between shoe frames.

The integrator contains two sub-processes running concurrently, using the Boost ASIO library, which is an open-source, cross-platform $\mathrm{C}++$ library for network programming and provides a framework for asynchronous function execution like threading. The two main sub-processes are a TCP data server and a Butterworth filter.

The TCP data server handles the connections from the clients (the shoevision processes) and parses the data that they send. When any client sends its data, the TCP server copies that data into a buffer accessible to both the TCP server and the Butterworth filter, overwriting any data it may have already contained. The Boost ASIO prevents the two concurrent sub-processes from writing to the buffer simultaneously.

The Butterworth filter runs at a fixed rate of $50 \mathrm{~Hz}$. At each time-step it uses each value in the shared buffer to update each value's own Butterworth filter. Finally, all

\footnotetext{
${ }^{6}$ In the sense that it minimizes the sum-square-difference error of the coordinates it transforms.
} 
output values are logged. ${ }^{7}$

The Butterworth filter is a special kind of low pass filter. It has the effect of smoothing the data, which is necessary for three reasons:

1. The integrator receives measurements from both shoes. Ideally, those two measurements will be identical, but in practice there will be some difference between the two. This difference causes sharp discontinuities every time the source of data switches from one shoe to the other.

2. Occasionally, an invalid solution may be sent to the integrator. The Butterworth filter reduces the effect of single anomalous solutions.

3. Since the camera image is noisy, the solutions produced from it will also contain noise. The Butterworth filter reduces the noise in the filtered solution.

The exact kind of low-pass filter used to smooth the data is not critical. A thirdorder Butterworth was chosen as a reasonable trade off between simplicity and cut-off frequency and because it has a flat frequency response. Since there is not a particular frequency to be removed, a sharp frequency cut-off is not necessary.

The Butterworth filter is implemented in its 3rd-order discrete time form, as shown below, with a cutoff frequency, $\omega_{c}$, of $9 \mathrm{~Hz}$.

$$
y_{k}=a_{0} x_{k}+a_{1} x_{k-1}+a_{2} x_{k-2}+a_{3} x_{k-3}-b_{1} y_{k-1}-b_{2} y_{k-2}-b_{3} y_{k-3},
$$

where

\footnotetext{
${ }^{7}$ Just before logging, the unit quaternion used to represent the shoe's orientation is re-normalized as the filtering process does not hold the norm of the quaternion constant.
} 


$$
\begin{aligned}
c & =\frac{2}{T \omega_{c}} \\
b_{0} & =c^{3}+2 c^{2}+2 c+1 \\
b_{1} & =\left(-3 c^{3}-2 c^{2}+2 c+3\right) / b_{0} \\
b_{2} & =\left(3 c^{3}-2 c^{2}-2 c+3\right) / b_{0} \\
b_{3} & =\left(-c^{3}+2 c^{2}-2 c+1\right) / b_{0} \\
a_{0} & =1 / b_{0} \\
a_{1} & =3 / b_{0} \\
a_{2} & =3 / b_{0} \\
a_{3} & =1 / b_{0} .
\end{aligned}
$$

In this implementation, $x_{k}$ is the raw input at time-step $k$, and $y_{k}$ is the filtered output at time-step $k$.

\subsection{Chapter Summary}

Each Raspberry Pi runs a process called shoevision which handles the image capture and processing for that shoe. The result of each shoevision process is sent to the integrator process which runs on the left shoe. The integrator process combines the results from both shoes, applies a smoothing filter, and logs the filtered results.

\subsubsection{Recommendations}

Although the software of the localization system functions as expected, there are three recommended areas of development, if further work is performed: 
1. Improve the FIND_SOLUTION algorithm so that zero false measurements are returned and a valid measurement is returned whenever possible.

2. Investigate replacing the integrator's Butterworth filter with a Kalman filter.

3. To reduce calculation time, identify and improve inefficient algorithms wherever possible. For example, replace Davenport's Q-method with the QUEST approximation. 


\section{Calibration}

This chapter describes the calibration of the localization system. Calibration is required for the normal operation of the system, and for the experiments that will be discussed in Chapter 5.

\subsection{Overview}

For the system to calculate $\boldsymbol{\Lambda}_{R L}$, a number of quantities must be measured and incorporated into the calculations. This process is commonly referred to as calibration. To help clarify the terminology used in this chapter, Figure 4.1 shows the coordinate systems referred to in this chapter. The calibration process makes use of the Optotrak system (introduced in Section 1.2.6), the Optotrak probe, a calibration board, and the localization system itself. The calibration board is a rigid board with a pattern of easily identifiable squares on one side and an Optotrak marker triplet on the other side. There are two coordinate systems fixed to the calibration board:

1. The pattern frame, $P$, which is aligned with the pattern of squares.

2. The board frame, $B$, which is aligned to the Optotrak marker triplet on the calibration board.

Each shoe of the localization system also has an Optotrak marker attached to its rear, 


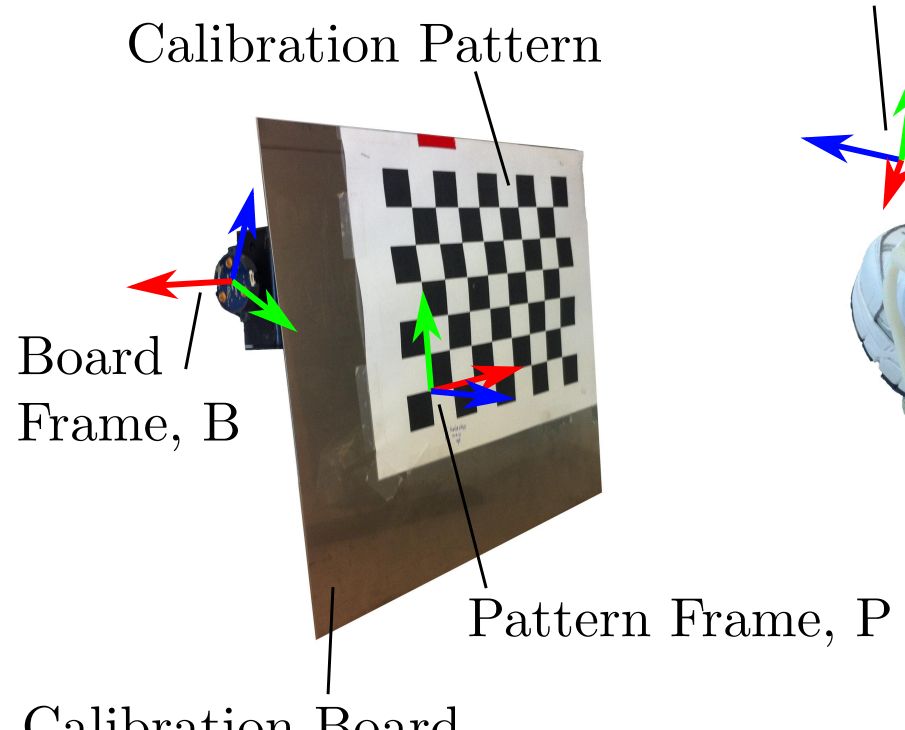

\section{Camera Frame, C}

Calibration Board

Figure 4.1.: Coordinate systems used in the calibration process.

used for calibration as well as the validation experiments of Chapter 5. Each shoe has two coordinate systems defined:

1. The camera frame, $C$, as described in Section 3.2.4, is aligned with the camera. Frame $C$ is simply an alias for frame $R$ or frame $L$ introduced in the previous chapter.

2. The rear frame, $O$, which is aligned with the Optotrak marker on the rear of the shoe.

The following quantities must be measured as part of the calibration process:

1. The coordinate system transformation from the pattern frame to the board frame. This transformation is not required for operation of the device, but is required for the following calibration steps (Section 4.2).

2. The intrinsic camera parameters used in the camera model (Section 4.3). 
3. The coordinate system transformation from the rear frame to the camera frame (Section 4.4).

4. The locations of the IR LED markers in the camera frame as well as the Fabrizio parameters derived from the marker locations (Section 4.5).

The four calibration procedures are performed for each shoe independently. Below, each procedure is described.

\subsection{Calibration Board Transformation}

The calibration board has two coordinate systems: the pattern frame given by the subscript $P$ and the board frame given by the subscript $B$. The transformation from the pattern frame to the board frame, $\boldsymbol{\Lambda}_{B P}$, is required for the later step of calculating $\boldsymbol{\Lambda}_{O C}$ described in Section 4.4.

\subsubsection{Measurement}

The Optotrak probe was used to measure the position of three points relative to the board frame: $\mathbf{v}_{B 1, B}, \mathbf{v}_{B 2, B}$, and $\mathbf{v}_{B 3, B}$. The three points that are measured are shown in Figure 4.2. The probe is held at each point for one second. Since the Optotrak records at a rate of $100 \mathrm{~Hz}, 100$ position measurements are made for each of the three

points. Therefore, the actual measurements will be: $\mathbf{v}_{B 1, B, n}, \mathbf{v}_{B 2, B, n}$, and $\mathbf{v}_{B 3, B, n}$ for $n=0 \ldots 99$.

The location of the three measured points lie at the origin, along the $\mathrm{x}$ axis and along the $y$ axis of the pattern frame. Thus their coordinates in the pattern frame are: 


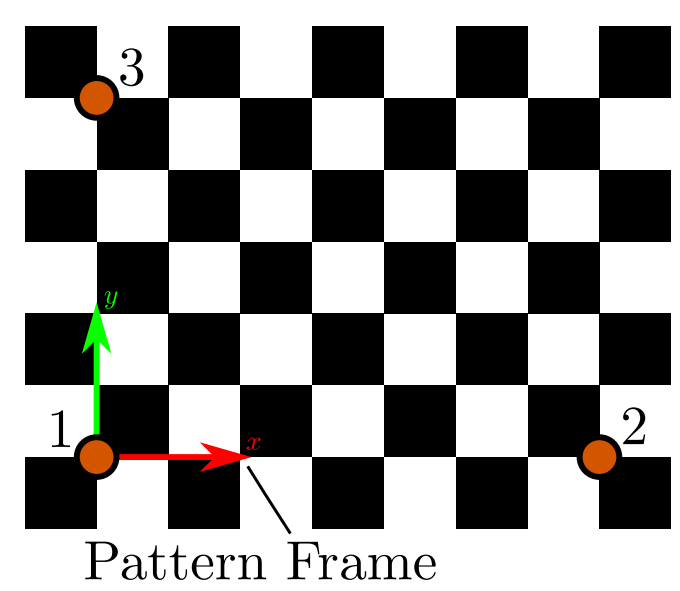

Figure 4.2.: The location of the three points measured by the Optotrak probe.

$$
\begin{aligned}
& \mathbf{v}_{P 1, P}=\left(\begin{array}{lll}
0 & 0 & 0
\end{array}\right) \\
& \mathbf{v}_{P 2, P}=d_{l}\left(\begin{array}{lll}
7 & 0 & 0
\end{array}\right) \\
& \mathbf{v}_{P 3, P}=d_{w}\left(\begin{array}{lll}
0 & 5 & 0
\end{array}\right),
\end{aligned}
$$

where $d_{l}$ and $d_{w}$ are the side lengths of a single black square along the length and width of the pattern respectively. ${ }^{1}$ The numbers 7 and 5 come from the number of squares between the measured points. The values of $d_{l}$ and $d_{w}$ will be determined from the Optotrak measurements.

\subsubsection{Data Processing}

Since the coordinates of the three points are known in both the pattern frame and the board frame, it is possible to calculate the coordinate system transformation from the pattern frame to the board frame using Davenport's Q-Method as was described in Section 3.2.5.

\footnotetext{
${ }^{1}$ The lengths $d_{l}$ should equal $d_{s}$ if the black squares are indeed square, but that assumption was not made here in case the calibration pattern was not printed with a perfect aspect ratio.
} 
First, the Optotrak measurements were averaged to estimate the coordinates of the points in the board frame:

$$
\begin{aligned}
& \mathbf{v}_{B 1, B}=\frac{1}{100} \sum_{n=0}^{99} \mathbf{v}_{B 1, B, n} \\
& \mathbf{v}_{B 2, B}=\frac{1}{100} \sum_{n=0}^{99} \mathbf{v}_{B 2, B, n} \\
& \mathbf{v}_{B 3, B}=\frac{1}{100} \sum_{n=0}^{99} \mathbf{v}_{B 3, B, n} .
\end{aligned}
$$

Next, $\mathbf{v}_{B 1, B}, \mathbf{v}_{B 2, B}$, and $\mathbf{v}_{B 3, B}$ were used to calculate the coordinates of the $x$ and $y$ axes of the pattern frame as measured in the board frame:

$$
\begin{gathered}
\hat{\mathbf{x}}_{P, B}=\frac{\mathbf{v}_{B 2, B}-\mathbf{v}_{B 1, B}}{\left\|\mathbf{v}_{B 2, B}-\mathbf{v}_{B 1, B}\right\|} \\
\hat{\mathbf{y}}_{P, B}=\frac{\mathbf{v}_{B 3, B}-\mathbf{v}_{B 1, B}}{\left\|\mathbf{v}_{B 3, B}-\mathbf{v}_{B 1, B}\right\|} .
\end{gathered}
$$

The coordinates of the same axes as measured in pattern frame are simply:

$$
\begin{aligned}
\hat{\mathbf{x}}_{P, P} & =\left(\begin{array}{lll}
1 & 0 & 0
\end{array}\right) \\
\hat{\mathbf{y}}_{P, P} & =\left(\begin{array}{lll}
0 & 1 & 0
\end{array}\right) .
\end{aligned}
$$

The coordinates of the $x$ and $y$ axes in the two frames were then used as the inputs to the Q-method to find the rotation from pattern frame to board frame: $\mathbf{q}_{B P}$. The translation from pattern frame to board frame is simply the coordinates of the first measured point: $\mathbf{v}_{B 1, B}$. Thus the coordinate system transformation from pattern 
frame to board frame is:

$$
\boldsymbol{\Lambda}_{B P}=\left(\mathbf{v}_{B 1, B}, \mathbf{q}_{B P}\right) .
$$

Finally, the side lengths of the black squares, $d_{l}$ and $d_{w}$, were calculated using:

$$
\begin{aligned}
d_{l} & =\frac{1}{7}\left\|\mathbf{v}_{B 2, B}-\mathbf{v}_{B 1, B}\right\| \\
d_{w} & =\frac{1}{5}\left\|\mathbf{v}_{B 3, B}-\mathbf{v}_{B 1, B}\right\| .
\end{aligned}
$$

\subsubsection{Results}

The result of this calibration step are:

1. $\boldsymbol{\Lambda}_{B P}$, the coordinate system transformation from the pattern frame to the board frame; and

2. $d_{l}$ and $d_{w}$, the side lengths of the black squares of the calibration pattern.

\subsection{Camera Calibration}

The camera model, as was discussed in Section 3.2.4, uses the 23 parameters that were shown in Table 3.1. These parameters, which will be given by $\mathbf{P} \in \mathbb{R}^{23}$, must be determined after the camera is assembled. The camera model, which transforms a point from image frame $(I)$ to a direction in camera frame $(C)$ using $\mathbf{P}$ will be given by the function $f_{c}$ :

$$
\hat{\mathbf{v}}_{C}=f_{C I}\left(\mathbf{P}, \mathbf{v}_{I}\right) .
$$




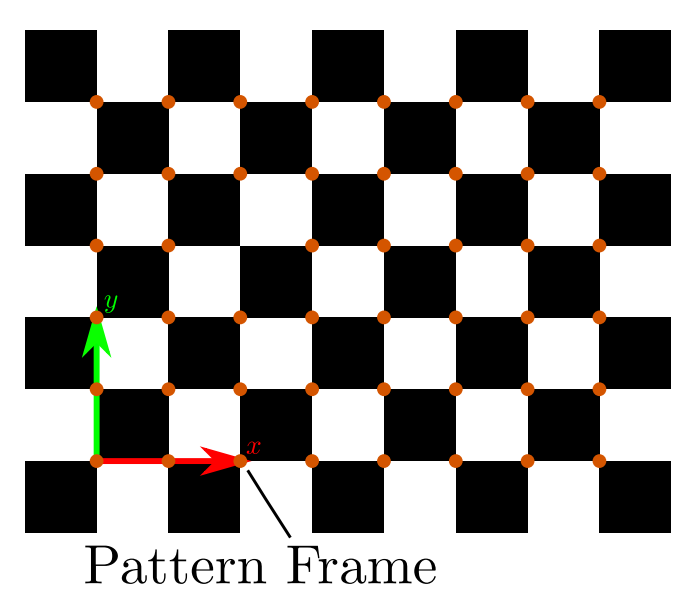

Figure 4.3.: The location of the corners in the calibration pattern. The corners are given by the orange circles.

The camera calibration was performed by capturing images of the calibration pattern. An initial estimate of $\mathbf{P}$ is used to calculate where the calibration pattern would appear in the image, which can be compared to where it actually appeared in the image. A numerical optimization routine was then used to alter the estimate of $\mathbf{P}$ until the model's predictions closely matched what was seen in the captured images.

\subsubsection{Measurement}

The shoe's camera captures images of the calibration pattern shown in Figure 4.1 from a variety of positions and orientations. The calibration pattern is positioned so that every region in the camera's field of view contains the pattern in at least one image. At least 10 images were captured for each camera, and the calibration pattern was typically between $2 \mathrm{~cm}$ and $80 \mathrm{~cm}$ from the camera lens. The calibration pattern was held so that its face was approximately normal to the direction of the camera. 


\subsubsection{Data Processing}

The calibration pattern contains an array of black squares, as shown in Figure 4.3. The points where the black squares contact each other are called corners. The coordinates of each corner in the pattern frame are given by $\mathbf{v}_{P n, P}$ where $n$ is the $n$th corner in the pattern. The values of $\mathbf{v}_{P n, P}$ can be determined using:

$$
\mathbf{v}_{P n, P}=(n \bmod 7)\left(\begin{array}{c}
d_{l} \\
0 \\
0
\end{array}\right)+\left\lfloor\frac{n}{7}\right\rfloor\left(\begin{array}{c}
0 \\
d_{w} \\
0
\end{array}\right),
$$

where $d_{l}$ and $d_{w}$ are the side lengths of the black squares found in Section 4.2, and 7 is the number of corners in the $x$ direction. The expression $(n \bmod 7)$ refers to $n$

modulo 7 from modular arithmetic. The expression $\left\lfloor\frac{n}{7}\right\rfloor$ means $\frac{n}{7}$ rounded down to the nearest integer.

At the instant each image is taken, there is an unknown coordinate system transformation from the pattern frame to the camera frame, given by $\boldsymbol{\Lambda}_{C P, i}$, where $i$ indicates the $i$ th image captured. The coordinates of each pattern corner $n$ in image $i$ then are given by:

$$
\mathbf{v}_{C n, C, i}=\Lambda_{C P, i}\left(\mathbf{v}_{P n, P}\right) .
$$

Since the camera model transforms points in the image frame to directions in the camera frame, the coordinates of the pattern corners in the image frame must be given using the inverse of the camera model, $f_{c}^{-1}$ :

$$
\mathbf{v}_{I n, I, i}=f_{C I}^{-1}\left(\mathbf{P}, \mathbf{v}_{C n, C, i}\right),
$$

where the inverse camera model is calculated by numerically solving the following 
equation for $\mathbf{v}_{I n, I, i}$ :

$$
0=\frac{\mathbf{v}_{C n, C, i}}{\left\|\mathbf{v}_{C n, C, i}\right\|}-f_{C I}\left(\mathbf{P}, \mathbf{v}_{I n, I, i}\right)
$$

From the captured image it is possible to find $\mathbf{v}_{I n, I, i}$, the coordinates of the pattern corners in the image frame. This is performed automatically using calibration pattern processing routines from the open-source OpenCV image processing library. Each corner located in the image provides a set of equations (from Equation 4.17) where $\mathbf{v}_{P n, P}$ and $\mathbf{v}_{I n, I, i}$ are known, but $\boldsymbol{\Lambda}_{C P, i}$ for each image $i$ and $\mathbf{P}$ are not known. Given enough pattern corners, and thus equations, it is possible to numerically solve for the unknowns. Because of the noise in the coordinate measurements, the problem becomes one of minimization. That is, the unknowns are calculated to minimize the error for all pattern corners, $n$, and each image, $i$ :

$$
e_{c}=\sum_{n} \sum_{i}\left\|\mathbf{v}_{I n, I, i}-f_{C I}^{-1}\left(\mathbf{P}, \boldsymbol{\Lambda}_{C P, i}\left(\mathbf{v}_{P n, P}\right)\right)\right\|^{2} .
$$

To compute a numerical solution, it is necessary to begin with a good initial estimate of the solution. The camera model was initially estimated as having the following simplifications:

1. Parameters $u_{0}, v_{0}$ are the coordinates of the center of the image.

2. Parameters $S_{1}, S_{2}$ are calculated from the camera's sensor size and resolution given in Table 2.2.

3. Parameter $D_{1}$ was estimated from the camera's measured field of view and radius of the circle of projection.

4. All other camera model parameters were initialized as small but non zero numbers to help with the numerical optimization. 
The pattern frame coordinate system transformations are estimated by first identifying the four outside corners of the pattern in the image. Then using the simplified camera model just described, a process exactly the same as described in Section 3.2.5 was followed to find the coordinate system transformation from the calibration pattern, but the coordinates of the four corners are used instead of the LED markers.

\subsubsection{Results}

The results of the minimization are:

1. $\mathbf{P}$, the 23 camera model parameters; and

2. $\boldsymbol{\Lambda}_{C P, i}$, the coordinate system transformations from the pattern frame to the camera frame for each image $i$.

The numerical values of $\mathbf{P}$ for both shoes can be found in Appendix B.

\subsection{Rear Shoe Optotrak Marker Transformation}

To compare the measurements made by the localization system with those made by the Optotrak, it is necessary to know $\boldsymbol{\Lambda}_{C O}$, the coordinate system transformation from the rear frame to the camera frame on each shoe.

To calculate $\boldsymbol{\Lambda}_{C O}$, the calibration pattern was used as an intermediate frame. When images were captured for the purpose of the camera calibration, the Optotrak also recorded the transformation from the board frame to the rear frame for each image $i$ : $\boldsymbol{\Lambda}_{O B, i}$, which are both defined by Optotrak marker triplets. From the camera calibration process in Section 4.3, the transformation from the pattern frame to the camera frame, $\boldsymbol{\Lambda}_{C P, i}$, is given for each image $i$. Finally, from the first calibration step in Section 4.2, the transformation from pattern frame to board frame, $\boldsymbol{\Lambda}_{B P}$, was 
found. These three transformations $\left(\boldsymbol{\Lambda}_{O B, i}, \boldsymbol{\Lambda}_{C P, i}\right.$, and $\left.\boldsymbol{\Lambda}_{B P}\right)$ can be combined to get $\boldsymbol{\Lambda}_{C O}$. This might be computed simply using any image $i$ as:

$$
\boldsymbol{\Lambda}_{C O}=\boldsymbol{\Lambda}_{C P, i} \boldsymbol{\Lambda}_{B P}^{-1} \boldsymbol{\Lambda}_{O B, i}^{-1} .
$$

Instead, a more optimal solution is described below to take advantage of the measurements from multiple images.

\subsubsection{Measurements}

The Optotrak recorded $\boldsymbol{\Lambda}_{O B, i}$, the transformation from the board frame to the rear frame for each image $i$ captured in Section 4.3. Other inputs include $\boldsymbol{\Lambda}_{C P, i}$ and $\boldsymbol{\Lambda}_{B P}$, which are calculated in the two previous calibration procedures.

\subsubsection{Data Processing}

The optimal coordinate system transformation between the camera frame and the rear frame was found such that it minimizes the distance between pattern frames as measured by the Optotrak system and the camera in the previous calibration.

The transformation from the pattern frame to the camera frame, $\boldsymbol{\Lambda}_{C P, i}=\left(\mathbf{v}_{C P, i}, \mathbf{q}_{C P, i}\right)$, which is given can also be calculated using the Optotrak's measurements and the desired transformation $\boldsymbol{\Lambda}_{C O}$ :

$$
\boldsymbol{\Lambda}_{C P, i, o p t o}=\left(\mathbf{v}_{C P, i, o p t o}, \mathbf{q}_{C P, i, o p t o}\right)=\boldsymbol{\Lambda}_{C O} \boldsymbol{\Lambda}_{O B, i} \boldsymbol{\Lambda}_{B P} .
$$

The transformation $\boldsymbol{\Lambda}_{C O}$ was then calculated using a least squares numerical minimization routine that minimized $d_{C P}$, the difference between the pattern frame positions for all images: 


$$
d_{C P}=\sum_{i}\left\|\mathbf{v}_{R P, i, o p t o}-\mathbf{v}_{R P, i}\right\|^{2} .
$$

\subsubsection{Results}

The result of this process was $\boldsymbol{\Lambda}_{C O}$, the coordinate system transformation from the camera frame to the rear frame. When it is necessary to differentiate between the left and right shoes the follow notation will be used:

1. $\boldsymbol{\Lambda}_{R O_{R}}=\boldsymbol{\Lambda}_{C O}$, for the right shoe; and

2. $\Lambda_{L O_{L}}=\Lambda_{C O}$, for the left shoe.

\subsection{IR LED Marker Locations}

The locations of the IR LED markers in the camera frame are used in calculating $\boldsymbol{\Lambda}_{R L}$ as was described in Section 3.2.5. Additionally, the location of the markers relative to each other is used to calculate the parameters $L_{12}, k_{1}$, and $k_{2}$ used in Fabrizio's solution to the P4P problem, described in Section 3.2.5.

The position of the center of an LED marker cannot be measured directly with the Optotrak system because the center point is embedded within the structure of the LED. Instead, the outside surface of the LED was measured and the center point was calculated from the surface data. The location of each marker was initially calculated relative to the rear frame, and then transformed to the camera frame using $\boldsymbol{\Lambda}_{C O}$ found in the previous calibration procedure. 


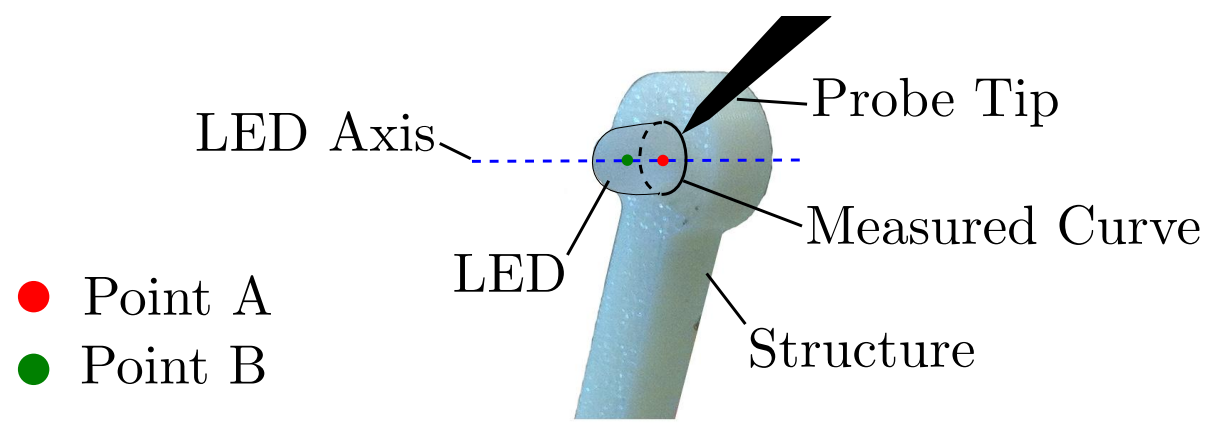

Figure 4.4.: Measurement of the IR LED locations. Point A lies in on the axis and in the plane of the measured curve. Point B, the center of the LED, lies on the axis 2.5 millimeters away from Point A.

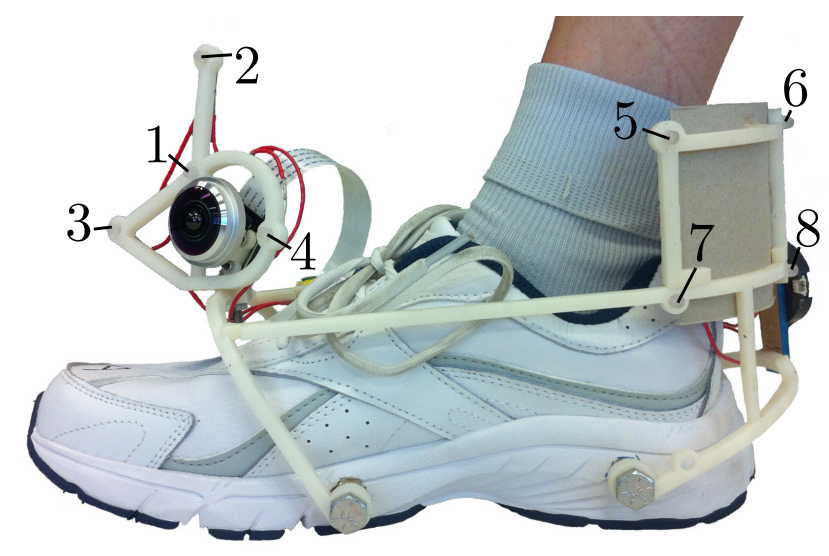

Figure 4.5.: The IR LED markers that must be measured during calibration.

\subsubsection{Measurement}

The Optotrak system recorded the position of the probe tip with respect to the rear frame. The probe tip was drawn along the perimeter of the each LED where it meets the skeletal structure, as shown in Figure 4.4. Because of movement and view constraints with the Optotrak system, the probe was only drawn along approximately a third of the total perimeter.

The result of the measurements was a list of points lying along the curve, with coordinates in the rear frame, for each marker shown in Figure 4.5: $\mathbf{v}_{O i, O, n}$, where $n$ indicates the LED marker index $(0-7)$, and $i$ indicates the index of the measurement for each marker. The $O$ indicates the rear frame. 


\subsubsection{Data Processing}

Using a numerical minimization routine, a circle was fitted to $\mathbf{v}_{O i, O, n}$ for all measurements $i$ of each marker $n$. The circle is represented by a center $\mathbf{v}_{O c, O, n}$, a normal direction $\hat{\mathbf{a}}_{O, n}$, and a radius $R_{n}$. The center of the circle, $\mathbf{v}_{O c, O, n}$ (Point $A$ in Figure 4.4), should lie along the axis of revolution of the LED. The direction $\hat{\mathbf{a}}_{O, n}$ points in the same direction as the LED's axis. The center of the LED $\mathbf{v}_{O n, O}$ (Point B in Figure 4.4) lies 2.5 millimeters along the same axis and was calculated using:

$$
\mathbf{v}_{O n, O}=\mathbf{v}_{O c, O, n}+2.5 \hat{\mathbf{a}}_{O, n}
$$

Next, the location of each marker $n$ was transformed to camera frame using:

$$
\mathbf{v}_{C n, C}=\boldsymbol{\Lambda}_{C O}\left(\mathbf{v}_{O n, O}\right)
$$

Finally, the parameters $L_{12}, k_{1}$, and $k_{2}$ used in Fabrizio's solution to the P4P problem, described in Section 3.2.5, were calculated for both the front and rear marker sets. Assuming that

$$
\begin{aligned}
& \mathbf{v}_{1}=\mathbf{v}_{C 0, C} \\
& \mathbf{v}_{2}=\mathbf{v}_{C 1, C} \\
& \mathbf{v}_{3}=\mathbf{v}_{C 2, C} \\
& \mathbf{v}_{4}=\mathbf{v}_{C 3, C},
\end{aligned}
$$

for the front marker set, and 


$$
\begin{aligned}
& \mathbf{v}_{1}=\mathbf{v}_{C 4, C} \\
& \mathbf{v}_{2}=\mathbf{v}_{C 5, C} \\
& \mathbf{v}_{3}=\mathbf{v}_{C 6, C} \\
& \mathbf{v}_{4}=\mathbf{v}_{C 7, C},
\end{aligned}
$$

for the rear marker set, the parameters are calculated as:

1. $L_{12}=\left\|\mathbf{v}_{1}-\mathbf{v}_{2}\right\| ;$ and

2. As described in [45], $K_{1}$ and $K_{2}$ are chosen to minimize the error of: $e_{f}=$ $\left\|\left(\mathbf{v}_{2}-\mathbf{v}_{1}\right) K_{1}+\left(\mathbf{v}_{4}-\mathbf{v}_{1}\right) K_{2}-\left(\mathbf{v}_{3}-\mathbf{v}_{1}\right)\right\|$.

\subsubsection{Results}

The results of this calibration, for each shoe, are

1. $\mathbf{v}_{C n, C}$, the locations of the LED markers in camera frame, and

2. the Fabrizio parameters $L_{12}, k_{1}$, and $k_{2}$ for both the front and rear marker set.

To match the notation used in the previous chapter:

1. $\mathbf{v}_{R n, R}=\mathbf{v}_{C n, C}$, for the right shoe; and

2. $\mathbf{v}_{L n, L}=\mathbf{v}_{C n, C}$, for the left shoe.

\subsection{Chapter Summary}

Four calibration procedures were performed for the localization system producing the following parameters for each shoe: 
1. $\mathbf{P}$, the 23 camera model parameters;

2. $\boldsymbol{\Lambda}_{C O}$, the coordinate system transformation from the rear frame to the camera frame;

3. $\mathbf{v}_{C n, C}$, the location of LED marker $n$ in the camera frame; and

4. $L_{12}, k_{1}$, and $k_{2}$, the Fabrizio parameters for both the front and rear marker set. A list of numerical values for these calibration results can be found in Appendix B. 


\section{Tests \& Characterization}

This chapter describes two experiments that were performed to test the concept of the localization system and characterize its performance.

\subsection{Experiment Overview}

Two experiments were performed to evaluate both the localization system and the integration of the localization system with the force sensing insole:

1. The short walk experiment was performed to determine how accurately the localization system measures the position and orientation of the shoes. This experiment is described in detail in Section 5.2.

2. The long walk experiment was performed to determine how well the localization system functions as a pedestrian tracking system, and is described in Section 5.3.

The short walk experiment was completed with 6 subjects, and the long walk experiment was completed with 5 subjects. Both experiments have been reviewed and received ethics clearance through the Carleton University Research Ethics Committee in accordance to the Tri-Council Policy Statement for Ethical Conduct for Research Involving Humans (Project Number: 101290). 


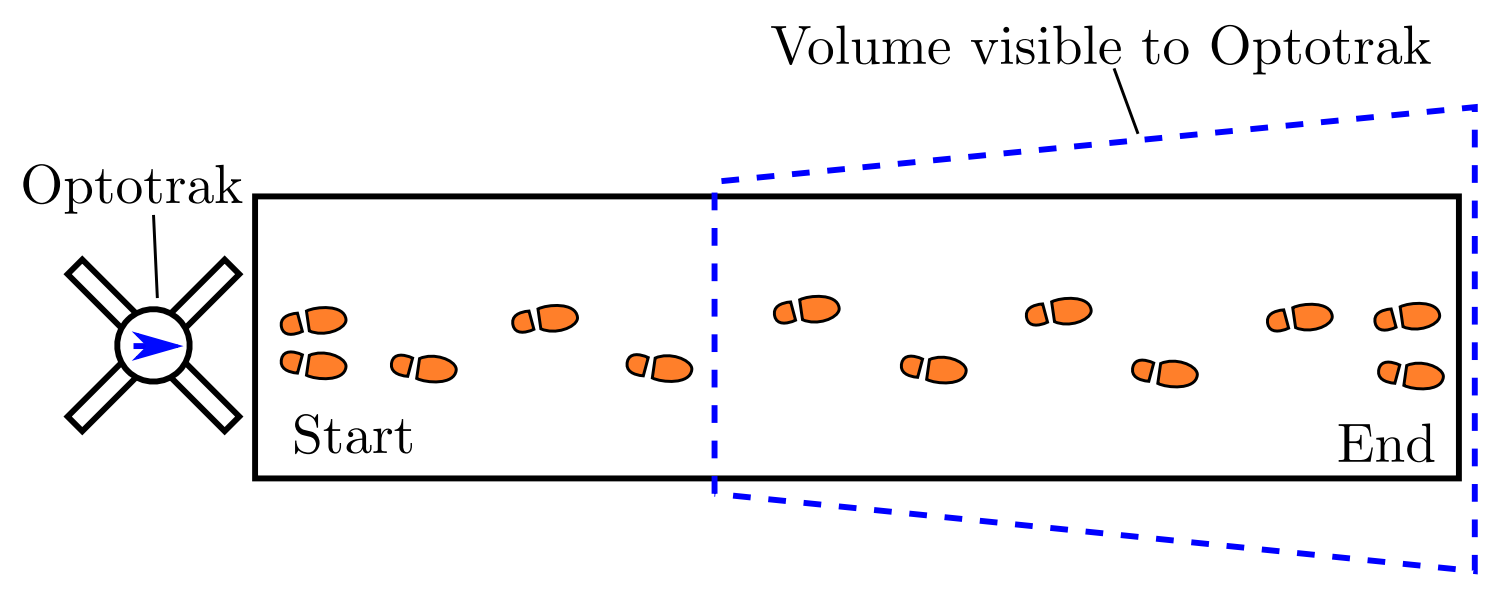

Figure 5.1.: Layout of the short walk experiment.

\subsection{Short Walk}

The short walk experiment was designed to test the accuracy of the system by comparing its measurements to those of the Optotrak Certus measurement system. While there are a number of sources of errors that can be accounted for or measured ahead of time, such as camera resolution and camera model accuracy, some sources of error can only be characterized in a real world setting. Many of these errors are discussed below, but examples include structural bending and streaking caused by fast shoe motion. This experiment allows us to characterize the combination of all the known sources of error and identify any unknown sources of error.

Subjects wearing the integrated system (that is, the localization system and force sensing insoles) were asked to walk normally for a short distance in a laboratory setting, as indicated in Figure 5.1. Figure 5.2 shows a photo of the actual experiment. Each subject was instructed to walk forward from the indicated start point to the end point then return to the start point, while pausing at the end point for three seconds. Each subject was asked to repeat this walk fifteen times: five times while walking normally, five times while walking slowly, and five times while walking quickly. 


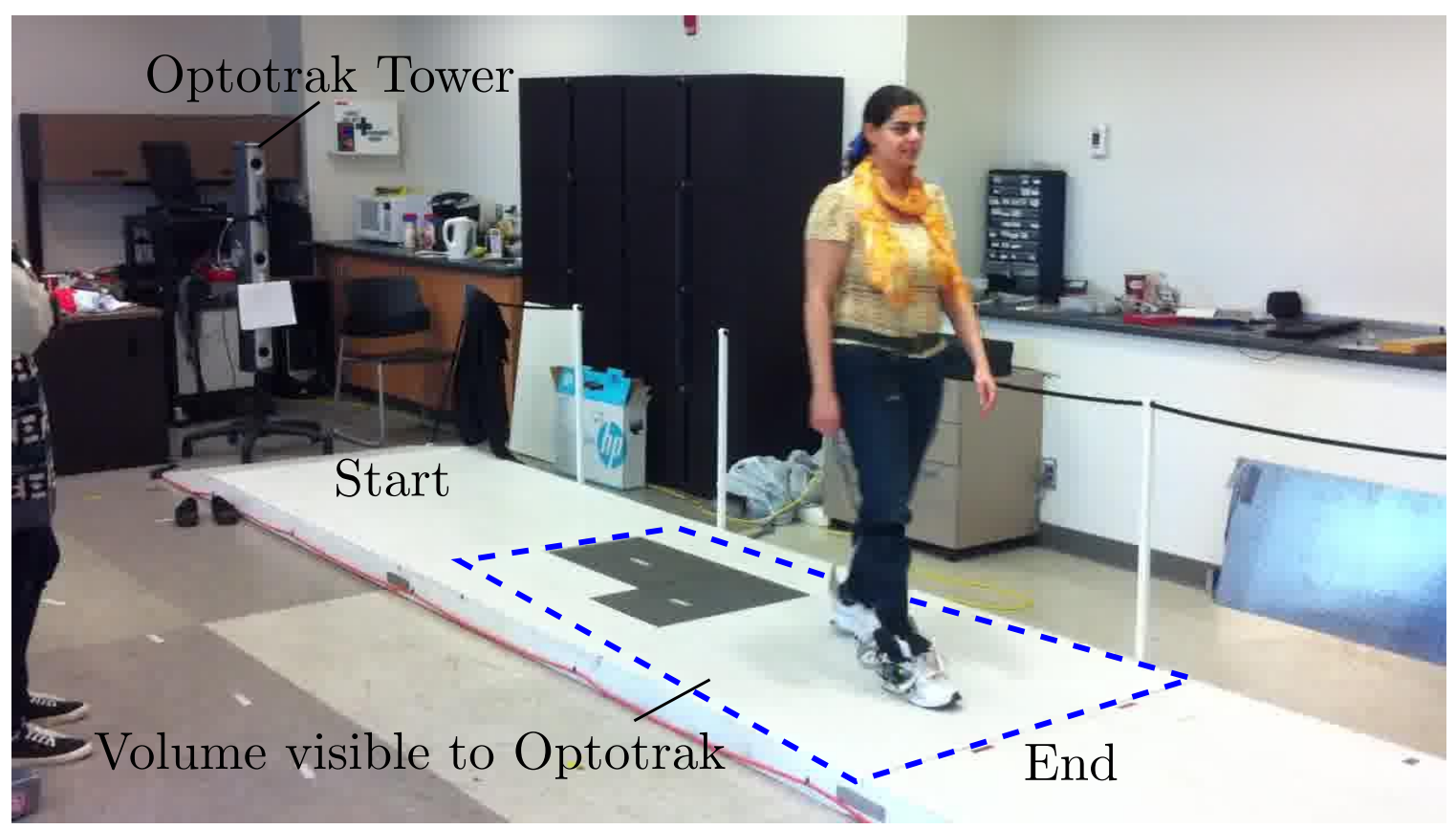

Figure 5.2.: Photo of the short walk experiment.

\subsubsection{Measurement}

During the experiment, the localization system continuously measured the coordinate system transformation from the right shoe camera frame to the left shoe camera frame: $\Lambda_{R L}$.

The Optotrak Certus recorded the position and orientation of the Optotrak markers on the rear of each shoe relative to the Optotrak tower: $\boldsymbol{\Lambda}_{T O_{L}}$ and $\boldsymbol{\Lambda}_{T O_{R}}$. The subscript $T$ indicates the frame of the Optotrak tower. $O_{L}$ and $O_{R}$ indicate the rear frame on the left and right shoe respectively. The Optotrak only made measurements while the subject was within the volume visible to the Optotrak, as indicated in Figure 5.1, and facing away from the Optotrak tower.

\subsubsection{Data Processing}

To determine how accurate the localization system is, it is necessary to calculate the error of each measurement it recorded. The error of each measurement is how much 
it differs from that of the Optotrak.

During the experiment, the Optotrak does not measure the same transformation as the localization system $\left(\boldsymbol{\Lambda}_{R L}\right)$. The coordinate system transformation between the two camera frames, $\boldsymbol{\Lambda}_{R L, o p t o}$, must be calculated from the Optotrak's measurements: $\boldsymbol{\Lambda}_{T O_{L}}$ and $\boldsymbol{\Lambda}_{T O_{R}}$. This was done by combining the Optotrak's measurements with the rear frame to camera frame transformations calculated in the calibration process (Section 4.4): $\boldsymbol{\Lambda}_{O_{L} L}$ and $\boldsymbol{\Lambda}_{O_{R} R}$. The desired transformation was calculated as:

$$
\boldsymbol{\Lambda}_{R L, o p t o}=\boldsymbol{\Lambda}_{O_{R} R}^{-1} \boldsymbol{\Lambda}_{T O_{R}}^{-1} \boldsymbol{\Lambda}_{T O_{L}} \boldsymbol{\Lambda}_{O_{L} L}
$$

The localization system measurements $\boldsymbol{\Lambda}_{R L}$ and the Optotrak measurements $\boldsymbol{\Lambda}_{R L, o p t o}$ were not synchronized in time. To compare them effectively, their time offset was determined by matching easily recognizable features in both signals, such as the standing still. This was performed semi-autonomously using routines that minimized the sum-of-squared-differences (SSD) of the major signal features. While necessary, this had the disadvantage of removing any actual time lag between the measurements, so characterization of the the time lag is not performed with this experiment.

Once the time offset was accounted for, the error between $\boldsymbol{\Lambda}_{R L}$ and $\boldsymbol{\Lambda}_{R L, \text { opto }}$ at a specific time ${ }^{1}$ was calculated with:

$$
\boldsymbol{\Lambda}_{\text {error }}=\left(\mathbf{v}_{\text {error }}, \mathbf{q}_{\text {error }}\right)=\boldsymbol{\Lambda}_{R L, \text { opto }}^{-1} \boldsymbol{\Lambda}_{R L}
$$

Instead of interpreting the 7 values that compose $\boldsymbol{\Lambda}_{\text {error }}$, two scalar measures of error were used: the translation error and the rotation error. Translation error, $d_{e}$, is defined as the magnitude of the translation of $\boldsymbol{\Lambda}_{\text {error }}$ :

\footnotetext{
${ }^{1}$ Since the exact measurements of these two signals will not match up, it was necessary to interpolate (linear) between $\boldsymbol{\Lambda}_{R L, \text { opto }}$ measurements to compare with the $\boldsymbol{\Lambda}_{R L}$ measurements.
} 


$$
d_{e}=\left\|\mathbf{v}_{\text {error }}\right\| .
$$

The rotation error, $r_{e}$, is the angle of rotation of $\boldsymbol{\Lambda}_{\text {error }}$ :

$$
r_{e}=2 \cos ^{-1}\left(\left|\mathbf{q}_{\text {error }, s}\right|\right),
$$

where the $s$ subscript indicates the scalar part of the unit quaternion.

The values of $d_{e}$ and $r_{e}$ were calculated for all available Optotrak measurements, which included a portion of walking and a portion of standing still, as will be shown in the next section.

\subsubsection{Results}

To provide an overall idea of the accuracy of the localization system, Figure 5.3 shows the frequency of the translation and rotation errors of the measurements. These frequency histograms were created using all of the available error measurements from all of the subjects, including the portions while the subject was standing still. As can be seen in Figure 5.4, when standing still the error is typically lower. The histograms indicate how likely it is that an error of a given size will occur. From the histograms the following summary conclusions can be made:

1. $90 \%$ of the translation errors are below $10.86 \mathrm{~cm}$,

2. $50 \%$ of the translation errors are below $2.60 \mathrm{~cm}$,

3. $90 \%$ of the rotation errors are below $25.19^{\circ}$, and

4. $50 \%$ of the rotation errors are below $6.14^{\circ}$.

If, in the worst case, an error of $11 \mathrm{~cm}$ and $25^{\circ}$ are made with the shoe localization system, this could result in a center of pressure on the order of $7 \mathrm{~cm}$ away from the 

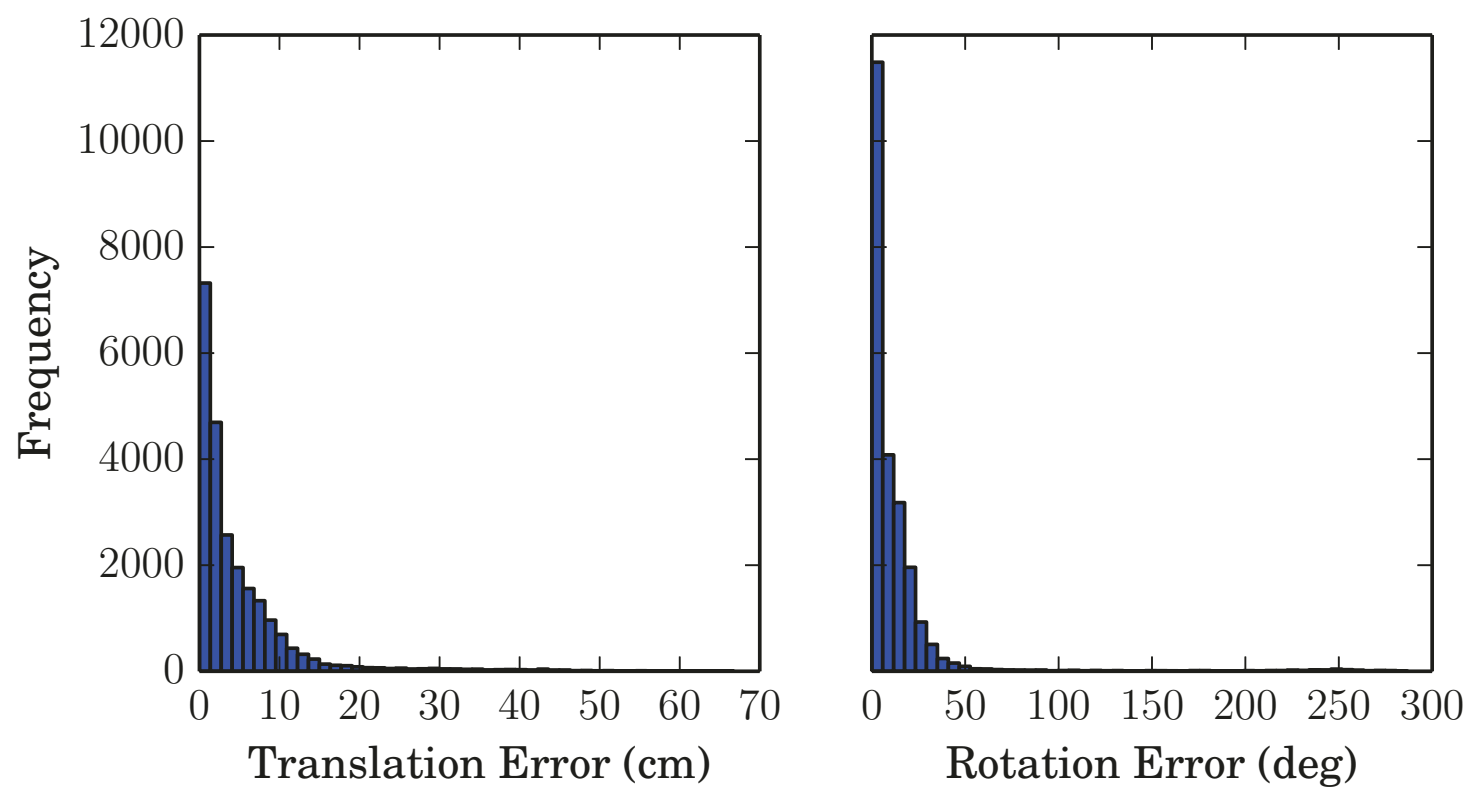

Figure 5.3.: Frequency of translation and rotation errors. The height of each bar indicates the number of measurements with an error in the range beneath that bar. The source data is a combination of all subjects.

true location.

To show how the errors vary over the course of the short walk, Figure 5.4 displays the translation error and the rotation error of a single subject for one short walk. In general, the error is larger and more varied while walking than the error while standing still. Figure 5.5 shows the average translation and rotation error during a single step for each walking speed. As can be seen, walking faster does incur a slightly higher error.

\subsubsection{Discussion}

Overall, the results presented in the previous section demonstrate that the localization system functions as intended, but as with any measurement system there is going to be some amount of error and noise in the measurements.

To help illustrate the sources of the error in the localization system measurements, 

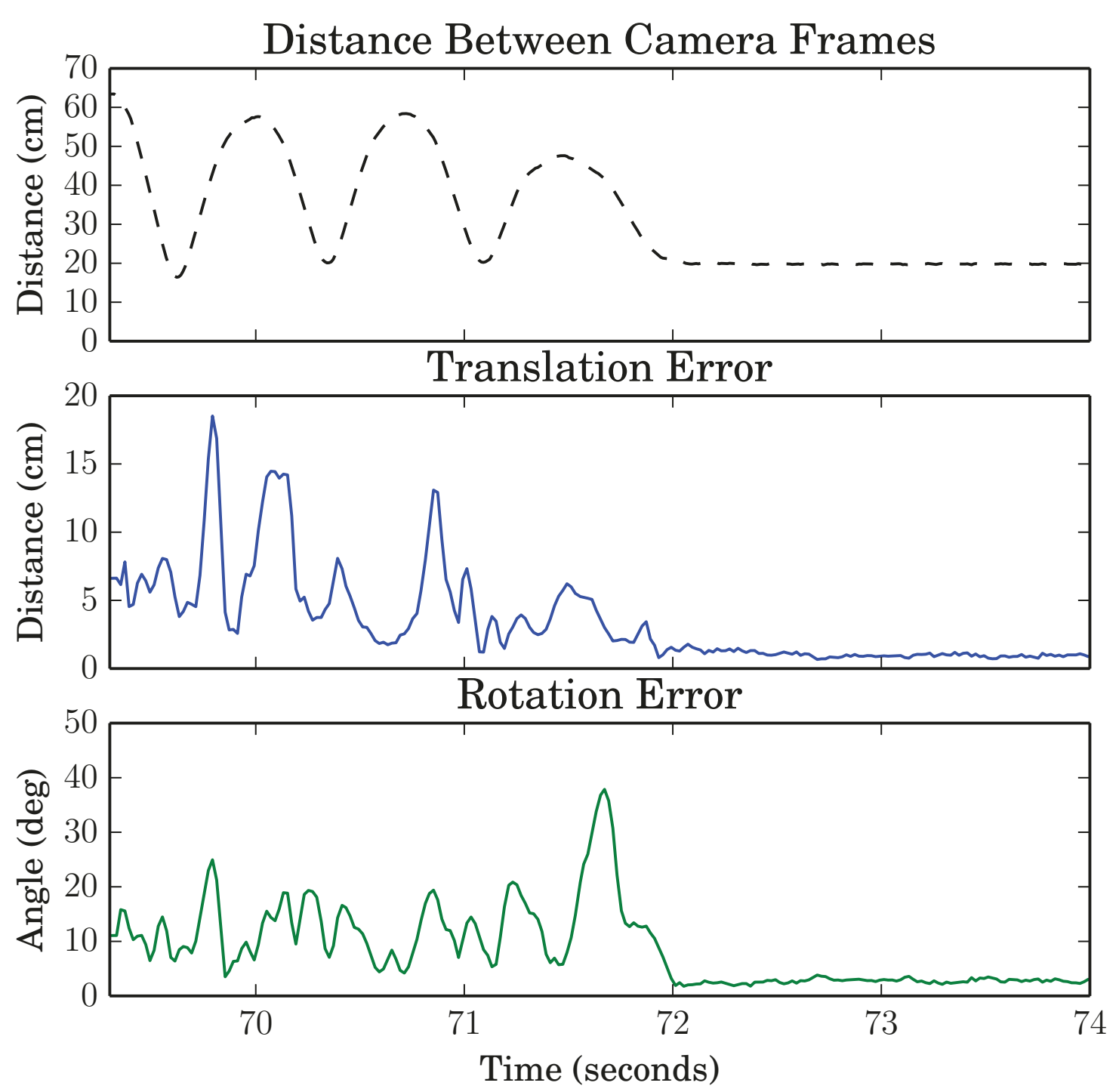

Figure 5.4.: Plots of translation and rotation error for a single subject during a single short walk. The distance between the camera frame measured by the Optotrak is given to indicate what the subject is doing. The first half of the time consists of walking normally, while the second half consists of standing still.

Figure 5.6 shows a plot of the distance between the shoes, as measured by the Optotrak and the localization system. As discussed in Chapter 3, each shoe makes its own measurements of the position and orientation of the shoes. These measurements are fed into the integrator where they are combined and filtered. The raw measurements of each shoe and the filtered results are all shown in Figure 5.6. 


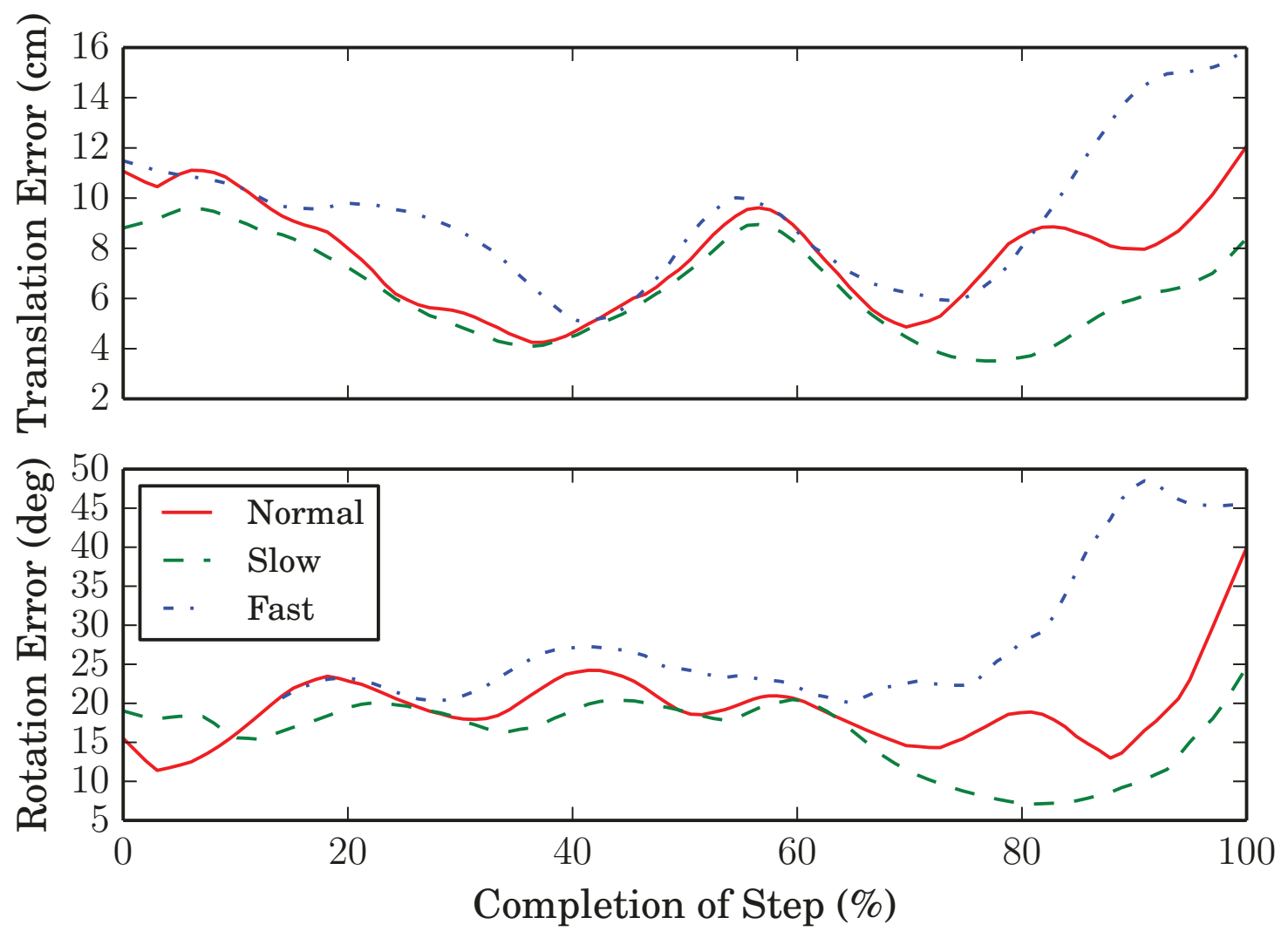

Figure 5.5.: The average translation and rotation errors during a single step while walking at a normal, slow, and fast pace. The data for each curve is an average of all the subjects. The single step begins with the left foot back and ends when the left foot is all the way forward.

With the localization system, errors can either be introduced in the raw measurements and carried into the filtered results or be introduced by the filtering process itself.

Most of the time, errors in the raw measurements tend to be relatively small, but less frequently very large anomalous measurements are produced, as shown in Figure 5.6. The anomalous measurements are a result of the shoevision process incorrectly identifying the markers during the procedure described in Section 3.2.3. This usually occurs when the relative orientation of the two shoes is too high (a small relative orientation is an assumption of the marker identification algorithm), but can also occur if the markers are obscured in a way that the algorithm does not consider, such as from a loose shoe lace. Sometimes, instead of producing a wrong solution, the 


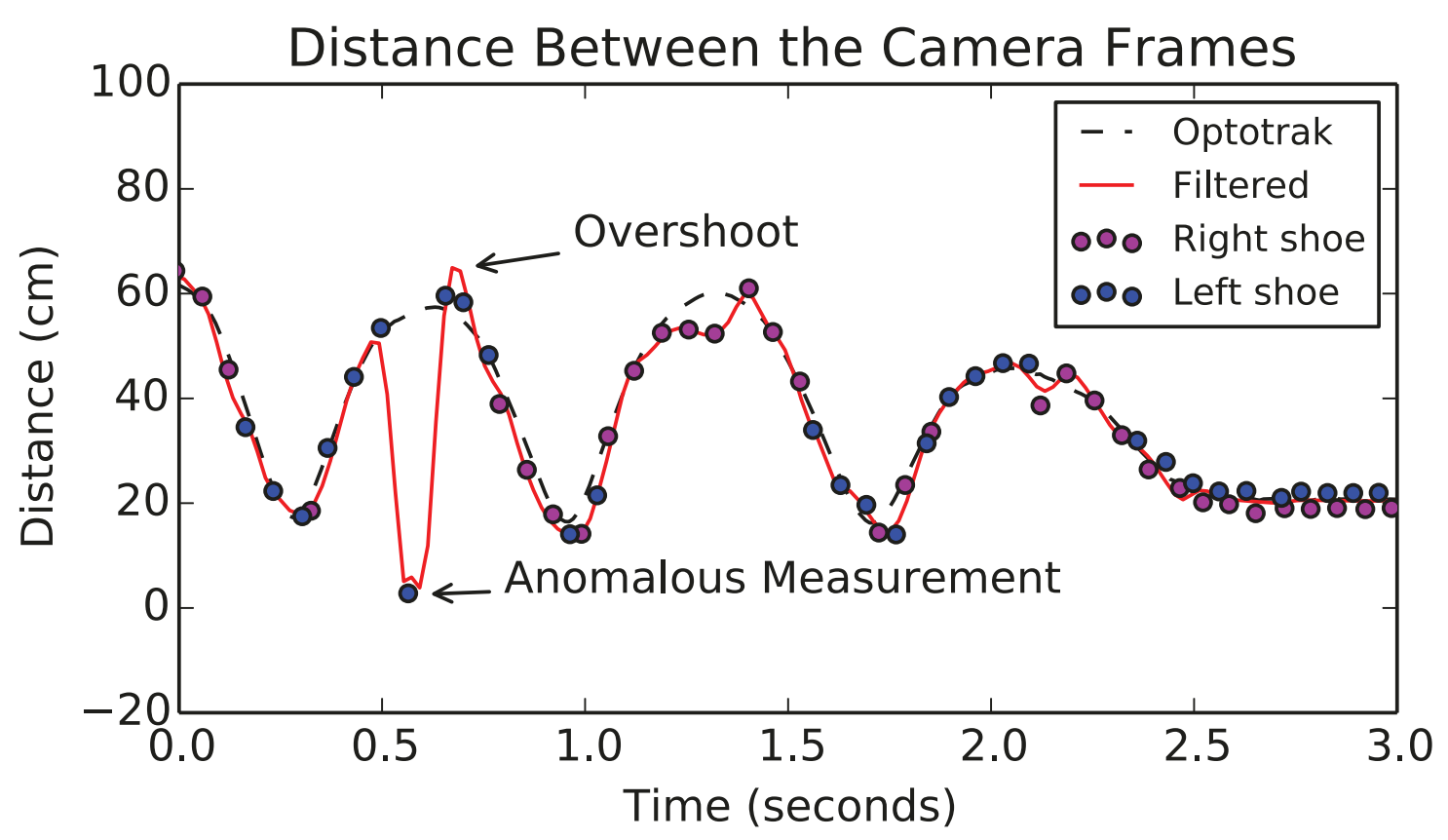

Figure 5.6.: Distance between the camera frames as measured by the Optotrak and the localization system, including the raw measurements made by each shoe.

shoevision process will produce no solution if it cannot identify a marker set. While this is preferable to a wrong solution, the lack of raw measurement can cause the filtered output to deviate from the correct solution until new raw measurements are available.

Besides the anomalous measurements, even the correct raw measurements will have some error. The sources of this error are a combination of the following factors:

1. Calibration errors. During the calibration process discussed in Chapter 4, a number of quantities are measured or calculated, such as the position of the LED markers and the parameters of the camera model. These measurements will have their own error which will contribute to the error seen in this chapter.

2. Camera Resolution. The fundamental limit to the device's accuracy is the camera sensor's resolution. A lower resolution results in less accurate measurements of direction, and thus the localization solution. 
3. LED marker distortion. If the markers are in movement as the camera captures images, they will streak to a small degree, making it more difficult to determine the center of the LED. Additionally, as the view angle to the LED varies, it may not always appear as a circle in the image, which means the center of the blob may not correspond with the center of the LED itself.

If the one or both of the shoes provide false measurements, the filtered output can significantly deviate from the correct path, as indicated in Figure 5.6. In addition to the initial deviation caused by the false measurement, when the next correct measurement is provided, the filtered output can experience over-shoot also causing error where otherwise correct measurements are made.

\subsection{Long Walk}

The purpose of the long walk experiment is to investigate the integrated system's performance as a pedestrian tracking system. Pedestrian tracking is not the goal of the BalanceAid project, but is a possible application of the technology.

While wearing the integrated system, the subject was asked to follow a preplanned path, as shown in Figure 5.7. The path was chosen to include a variety of turns in addition to straight paths. By combining the data from the localization system and the force sensing insoles, the locations of the wearer's footsteps were calculated relative to the wearer's starting position.

\subsubsection{Measurement}

During the experiment, the integrated system recorded the coordinate system transformation between the camera frames on the two shoes: $\boldsymbol{\Lambda}_{R L}$ and the magnitude of 


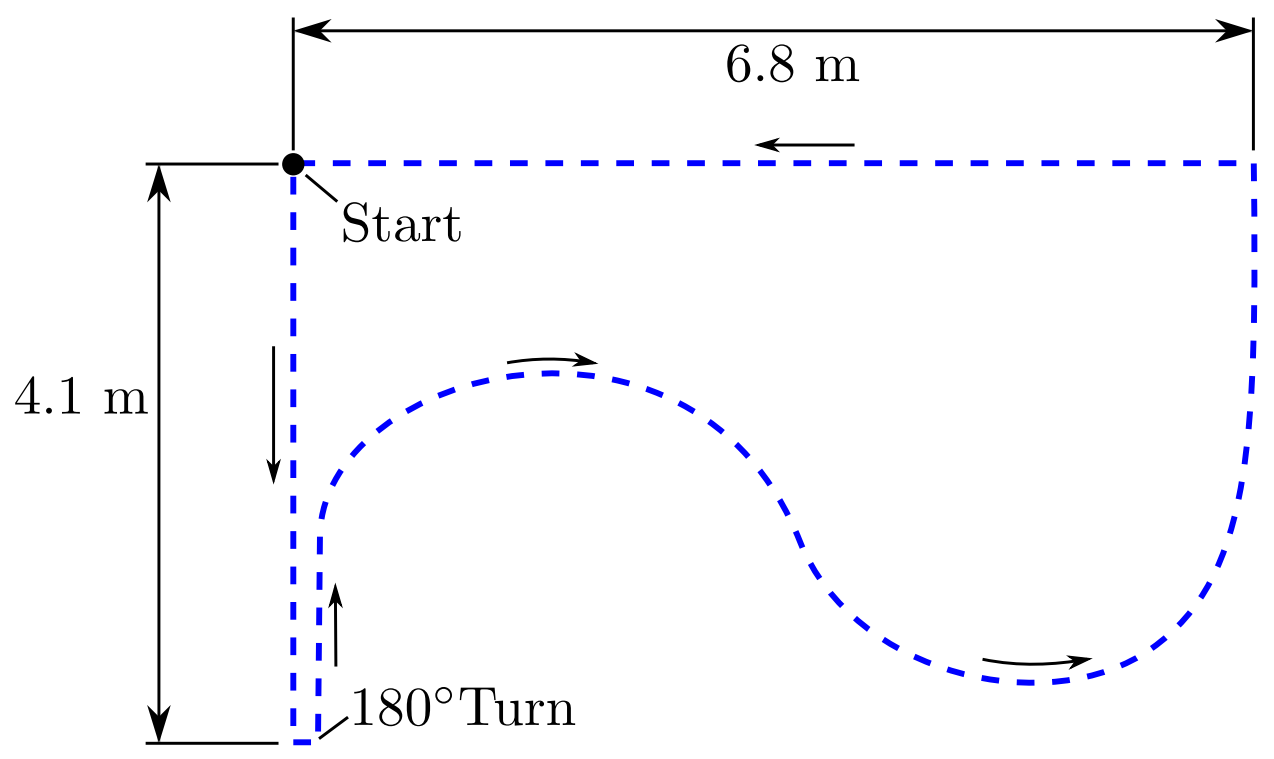

Figure 5.7.: Path followed by subjects during the long walk experiment.

the ground reaction force beneath the left foot, $F_{L}$, and the right foot, $F_{R}$. All of these values vary in time.

\subsubsection{Data Processing}

The tracking algorithm functions by appending together the measured $\boldsymbol{\Lambda}_{R L}$ of each step, resulting in a transformation from the camera frame of both shoes each time they contact the ground relative to the initial starting position.

While walking, the subject's weight shifts between $F_{L}$ and $F_{R}$. Every time $F_{L}$ and $F_{R}$ become equal, the transformation from the forward $(F)$ shoe to the rear $(E)$ shoe, $\boldsymbol{\Lambda}_{E F}$, is determined. If the left shoe is forward, then this is simply: $\boldsymbol{\Lambda}_{E F}=\boldsymbol{\Lambda}_{R L}$. If the right shoe is forward, then: $\boldsymbol{\Lambda}_{E F}=\boldsymbol{\Lambda}_{R L}^{-1}$. Ideally, it would be possible to simply combine the $\boldsymbol{\Lambda}_{E F}$ of each weight shift to arrive at the $n$th step of the subject: $\boldsymbol{\Lambda}_{\text {step }, n}=\boldsymbol{\Lambda}_{E F, 0} \boldsymbol{\Lambda}_{E F, 1} \boldsymbol{\Lambda}_{E F, 2} \ldots \boldsymbol{\Lambda}_{E F, n}$, but this simple procedure assumes that when one shoe is in contact with the ground, it remains completely fixed until the other shoe comes into contact with the ground. In practice, while one steps forward, the 
shoe in contact with the ground typically rolls forward from the heel to the toe. This would cause the estimated path followed by the subject to roll up, as if they were walking on the inside surface of a sphere rather than the flat surface of the ground.

To combat this path distortion, the three dimensional system is flattened into the two dimensional system that is the surface of the ground. To accomplish this, each shoe is provided with a new horizontal coordinate system denoted by $H$. The $H$ frame shares the same origin as the camera frame, but each $x$ axis points in the direction of the toes (ie, in the same direction as the shoe points), and its $z$ axis points up, normal to the sole of the shoe. Thus the $x y$ plane of the $H$ frame is parallel to the plane of the ground when the shoe is flat on the ground. The coordinate system transformation from camera frame to the horizontal frame is given by $\boldsymbol{\Lambda}_{H_{L} L}$ for the left shoe and $\boldsymbol{\Lambda}_{H_{R} R}$ for the right shoe.

Using the following procedure, the transformation $\boldsymbol{\Lambda}_{E F}$ is flattened such that the translation, $\mathbf{v}_{E F}$, is only in the $x y$ plane and the rotation, $\mathbf{q}_{E F}$, is only about the $z$ axis.

The translation of $\boldsymbol{\Lambda}_{E F}$ is flattened by orthographically projecting $\mathbf{v}_{E F}$ to the $x y$ plane, then changing its magnitude to be the same as $\mathbf{v}_{E F}$ :

$$
\mathbf{v}_{E F, \text { flat }}=\frac{\left\|\mathbf{v}_{E F}\right\| \mathbf{A v}_{E F}}{\left\|\mathbf{A v}_{E F}\right\|}
$$

where

$$
\mathbf{A}=\left(\begin{array}{lll}
1 & 0 & 0 \\
0 & 1 & 0 \\
0 & 0 & 0
\end{array}\right)
$$

This way, $\mathbf{v}_{E F, \text { flat }}$, keeps the same magnitude and horizontal direction as $\mathbf{v}_{E F}$, but now is parallel to the ground. 
The rotation of $\boldsymbol{\Lambda}_{E F}$ is flattened by applying a correction rotation, $\mathbf{q}_{f i x}$, that removes any rotation about the $x$ or $y$ axes, leaving only rotation about the $z$ axis:

$$
\mathbf{q}_{E F, f l a t}=\mathbf{q}_{f i x} \mathbf{q}_{E F}
$$

where

$$
\begin{aligned}
& \mathbf{q}_{f i x}=\left(\cos \left(\frac{1}{2} \theta\right), \hat{\mathbf{a}} \sin \left(\frac{1}{2} \theta\right)\right) \\
& \hat{\mathbf{a}}=\frac{\hat{\mathbf{z}}_{H_{F}, H_{E}} \times \hat{\mathbf{z}}_{H_{F}, H_{F}}}{\left\|\hat{\mathbf{z}}_{H_{F}, H_{E}} \times \hat{\mathbf{z}}_{H_{F}, H_{F}}\right\|} \\
& \theta=\cos ^{-1}\left(\hat{\mathbf{z}}_{H_{F}, H_{F}} \cdot \hat{\mathbf{z}}_{H_{F}, H_{E}}\right) \\
& \hat{\mathbf{z}}_{H_{F}, H_{F}}=\left(\begin{array}{lll}
0 & 0 & 1
\end{array}\right)^{T} \\
& \hat{\mathbf{z}}_{H_{F}, H_{E}}=\boldsymbol{\Lambda}_{H_{E} H_{F}}\left(\hat{\mathbf{z}}_{H_{F}, H_{F}}\right) \\
& \boldsymbol{\Lambda}_{H_{E} H_{F}}= \begin{cases}\boldsymbol{\Lambda}_{H_{R} R} \boldsymbol{\Lambda}_{R L} \boldsymbol{\Lambda}_{H_{L} L}^{-1} & \text { Left forward } \\
\boldsymbol{\Lambda}_{H_{L} L} \boldsymbol{\Lambda}_{R L}^{-1} \boldsymbol{\Lambda}_{H_{R} R}^{-1} & \text { Right forward }\end{cases}
\end{aligned}
$$

The rotation $\mathbf{q}_{f i x}$ is created by first calculating $\theta$, the angle between the $z$ axes of the rear and forward horizontal frames. This will be the angle of rotation. Second, the axis of rotation is the vector perpendicular to both $z$ axes. This rotation aligns the $z$ axes of the two horizontal frames, using the shortest route.

The flattened coordinate system transformation is:

$$
\boldsymbol{\Lambda}_{E F, f l a t}=\left(\mathbf{v}_{E F, f l a t}, \mathbf{q}_{E F, \text { flat }}\right)
$$

Finally, the coordinate system transformation from the $n$th step to the first step can 


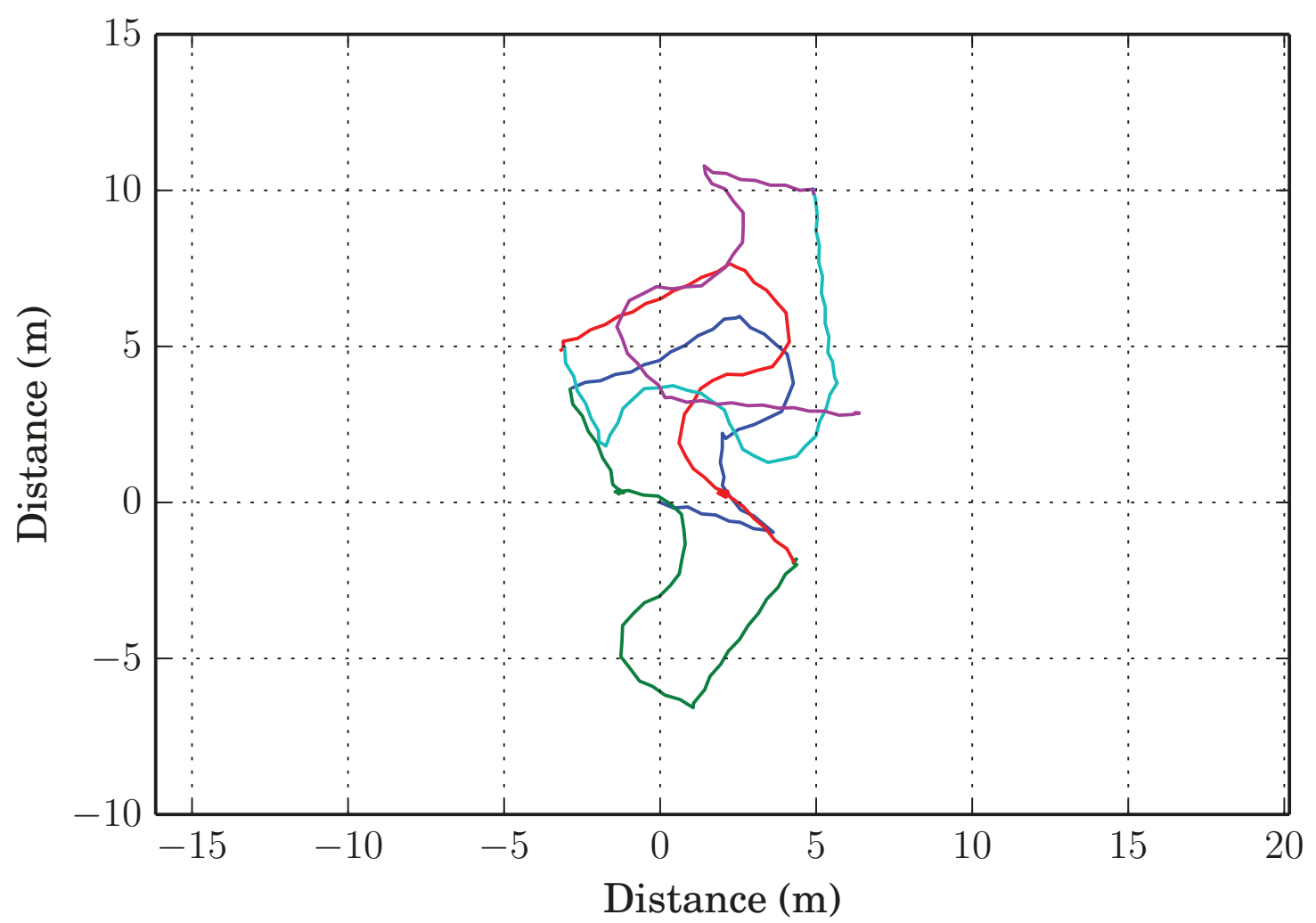

Figure 5.8.: The path of a subject estimated from the tracking algorithm. Starting point is at $(0,0)$. Each colour represents a different circuit.

be calculated by combining the transformations of each step:

$$
\boldsymbol{\Lambda}_{\text {step }, n}=\boldsymbol{\Lambda}_{E F, f l a t, 0} \boldsymbol{\Lambda}_{E F, \text { flat }, 1} \boldsymbol{\Lambda}_{E F, f l a t, 2} \ldots \Lambda_{E F, \text { flat }, n}
$$

The result, $\boldsymbol{\Lambda}_{\text {step }, n}=\left(\mathbf{v}_{\text {step }, n}, \mathbf{q}_{\text {step }, n}\right)$ contains the position and orientation of the forward shoe for step $\mathrm{n}$ relative to the starting position.

\subsubsection{Results}

The points $\mathbf{v}_{\text {step }, n}$ for all $n$ trace the path of the subject estimated by the tracking algorithm. An example of this path for one of the subjects is shown in Figure 5.8. This figure shows all five circuits made by the subject as one continuous path. To 


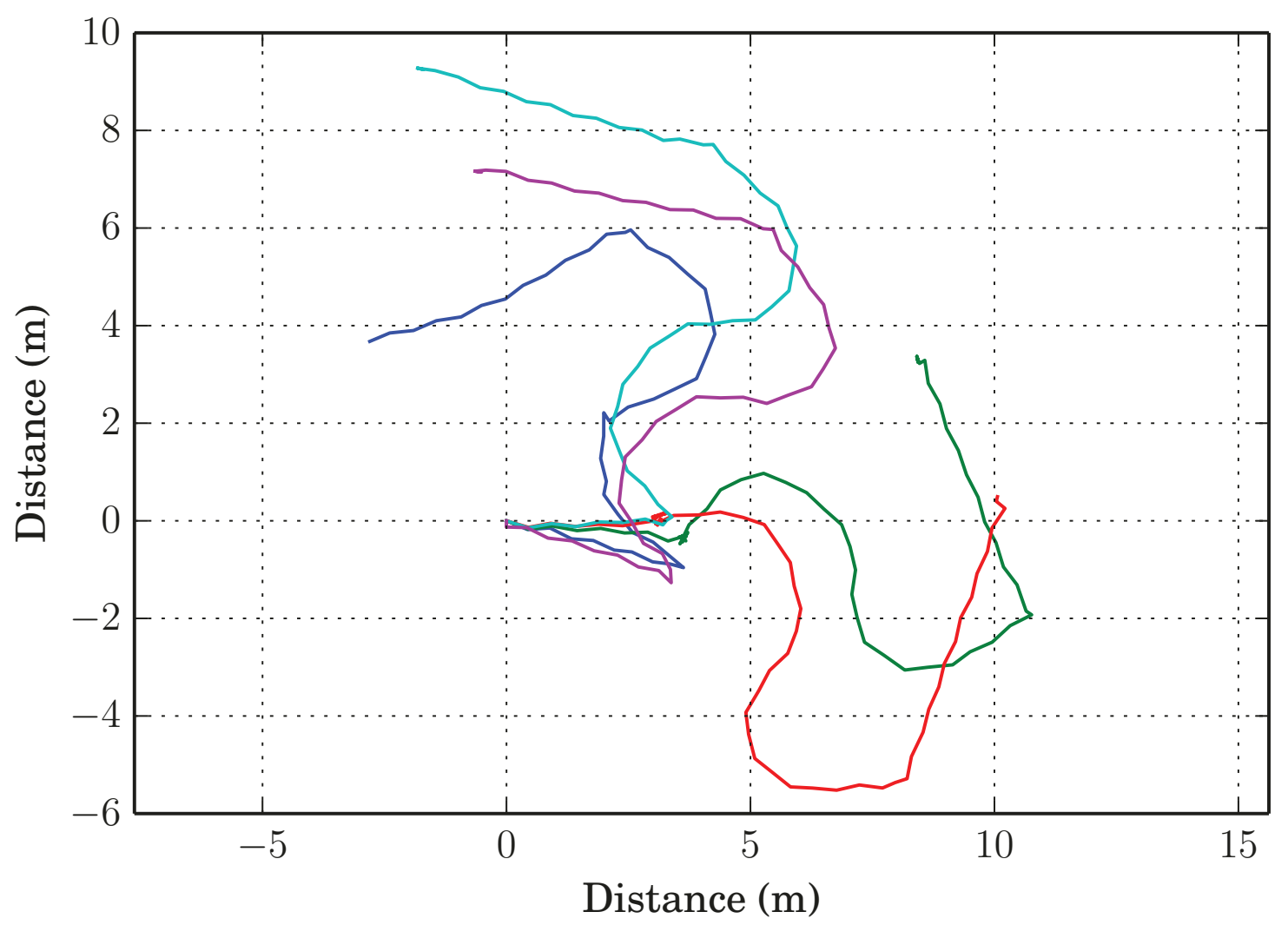

Figure 5.9.: The path of each circuit made by a subject estimated from the tracking algorithm. Starting point for each circuit is at $(0,0)$. Each colour represents a different circuit.

better show the variation between each path, Figure 5.9 shows each circuit starting from the same point and orientation.

Plots similar to Figure 5.9 for all subjects can be found in Appendix C. The estimated path shown in this section represented the cleanest and most accurate result among the other subject's estimated paths.

\subsubsection{Discussion}

Features of the true path, shown in Figure 5.7, can easily be seen in Figures 5.8 and 5.9 , but there also is significant deviation from the true path. Walking in a straight line is represented quite well, but the turns (particularly the $180^{\circ}$ turns), often result 


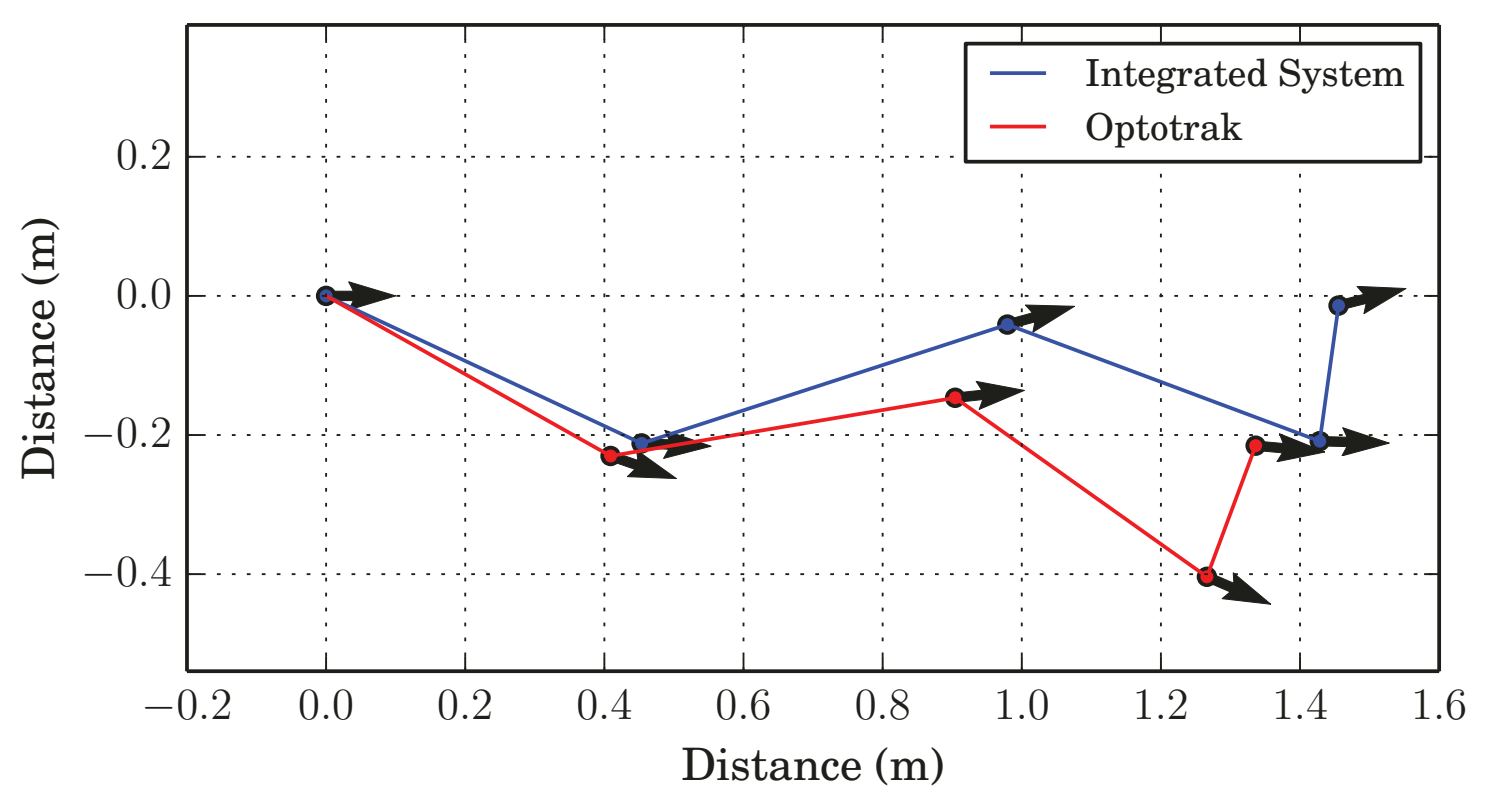

Figure 5.10.: The results of the tracking algorithm compared to the true steps as measured by the Optotrak.

in significant deviation. In Figure 5.9, it is clear that the $180^{\circ}$ turn is never estimated correctly, although it occasionally comes close. The main reason for major deviation to occur at the turns is a result of how the subjects would turn. When turning, the subjects would typically slide or rotate their feet while both were in contact with the ground. Since the tracking algorithm assumes that shoes in contact with the ground remain fixed, this results in the errors observed.

In addition to the errors introduced by foot slippage, all of the errors discussed in Section 5.2.4 directly contribute to the accuracy of the estimated path. For example, Figure 5.10 shows the results of the tracking algorithm when applied to the data from the previously discussed short walk experiment. This path includes just four steps, and the estimated path is compared to the actual path determined from the recorded Optotrak data. As can be seen, despite there being no turns, small errors in shoe orientation and position still accumulate significantly over the four steps.

Although interesting, the application of the BalanceAid system for pedestrian tracking 
seems limited when the feet are slipping (especially during sharp curves), therefore, an enhanced sensing strategy or slippage estimation should be investigated before this system becomes useful in this particular application.

\subsection{Chapter Summary}

From the short walk experiment:

1. The localization system was able to correctly calculate the position and orientation between the feet with $90 \%$ of the measurement errors being less than 10.85 $\mathrm{cm}$ and $25.19^{\circ}$.

2. The measurement error is larger and more varied during walking than standing still.

3. The walking pace does affect the average error, with a faster pace resulting in slightly higher error.

From the long walk experiment:

1. The path estimated from the localization system's measurements retained many of the features of the actual path, but overall deviated significantly from the actual path.

2. During turns, shoe slippage results in a very high error in the direction taken by the subject after the turn. 


\section{Conclusion}

The work presented in this thesis has resulted in the initial design and prototype of a self-contained wearable system capable of determining the relative position and orientation of the wearer's shoes. This system is the first attempt to use two shoe mounted cameras to measure the position and orientation of the shoes during the entire gait cycle in real time and using entirely on-board processing.

The design of this system completes a component of the longer term BalanceAid project, and much of the work in hardware selection and the design of the software architecture was designed for the BalanceAid system as a whole. This thesis also included the integration of the shoe localization system and the force sensing insole system, another component of the BalanceAid project.

Experiments were performed using 6 healthy subjects to characterize the performance of the localization system. The following conclusions were made from the experimental results:

1. The localization system was able to correctly calculate the position and orientation between the shoes with $90 \%$ of the measurement errors being less than $10.85 \mathrm{~cm}$ and $25.19 \mathrm{deg}$.

2. The measurement error is larger and more varied while the subjects were walking than standing still.

3. The walking pace does affect the average error, with a faster pace resulting in 
slightly higher error.

Overall, the experiments demonstrate that the concept behind the localization system is successful, but further development is required to eliminate anomalous measurements and reduce the susceptibility of the system to sources of error.

In addition to the previous experiment, the localization system integrated with the force sensing was applied to the challenge of pedestrian tracking. The path estimated from the system's measurements retained many of the features of the actual path, but overall deviated significantly from the actual path. The application of the BalanceAid system for pedestrian tracking seems limited when the feet are slipping (especially during sharp curves), therefore, an enhanced sensing strategy or slippage estimation should be investigated before this system becomes useful in this particular application.

\subsection{Recommendations for Future Work}

The following is a list of specific recommendations for improving the hardware design of the localization system:

1. It is recommended that smaller LED markers are used, perhaps even surface mounted LEDs.

2. The camera sensor should be sized to include the entire circle of projection of the fish-eye lens.

3. The on-board computer and camera module should be selected to achieve a higher framerate, such as 30 frames per second. It may be possible to stay with the Raspberry $\mathrm{Pi}$, as long as the lens/sensor are sized differently.

4. A new camera and marker set placement should be chosen to significantly reduce the fragility and complexity of the current skeletal structure. It may be 
necessary to depart from the on-shoe model and mount the camera elsewhere on the body such as the legs so that both feet are visible.

5. A bend sensor in the shoe's sole or insole should be included in the system to capture the amount of bend in the shoe while walking. This could help better capture measurement error to shoe bending, or possibly allow the front and rear marker set to not be riggidly fixed with respect to one another.

The following is a list of specific recommendations for improving the software design of the localization system:

1. Improve the FIND_SOLUTION algorithm so that zero false measurements are returned and a valid measurement is returned whenever possible.

2. Investigate replacing the integrator's Butterworth filter with a Kalman filter.

3. To reduce calculation time, identify and improve inefficient algorithms where ever possible. For example, replace Davenport's Q-method with the QUEST approximation.

The localization system was created as a component of the BalanceAid project. Thus the next step to take with the localization system is to integrate it fully it with the remaining BalanceAid components, and use the BalanceAid system in future experiments to determine its effectiveness. Experiments are planned with healthy but fatigued or distracted subjects in order to assess the effect of the system on subjects with reduced balance. 


\section{References}

[1] Public Health Agency of Canada. The Safe Living Guide - A guide to home safety for seniors. Tech. rep. Ottawa, ON: Public Health Agency of Canada, 2011 (cit. on p. 1).

[2] SMARTRISK. The Economic Burden of Injury in Canada. Tech. rep. Toronto, ON: SMARTRISK, 2009 (cit. on p. 1).

[3] Conrad Wall III and Marc S. Weinberg. "Balance prostheses for postural control". In: IEEE Engineering in Medicine and Biology Magazine April (2003). URL: http : / / ieeexplore . ieee .org / xpls / abs\%5C_all . jsp? arnumber = 1195701 (cit. on p. 1).

[4] Aruni U. Alahakone and S. M. N. Arosha Senanayake. "A Real-Time System With Assistive Feedback for Postural Control in Rehabilitation". In: IEEE/ASME Transactions on Mechatronics 15.2 (2010), pp. 226-233. DOI: 10.1109/TMECH. 2010.2041030 (cit. on pp. 1, 15).

[5] Justin R. Davis et al. "Trunk sway reductions in young and older adults using multi-modal biofeedback." In: Gait $\&$ Posture 31.4 (Apr. 2010), pp. 465-72. ISSN: 1879-2219. DOI: 10.1016/j.gaitpost. 2010.02.002. URL: http://www . ncbi.nlm.nih.gov/pubmed/20206528 (cit. on pp. 1, 3, 15).

[6] Edith V. Sullivan et al. "Postural sway reduction in aging men and women: relation to brain structure, cognitive status, and stabilizing factors". In: Neurobiology of Aging 30.5 (May 2009), pp. 793-807. ISSN: 1558-1497. DOI: 10.1016/j. neurobiolaging. 2007.08.021. URL: http://www. pubmedcentral.nih.gov/ articlerender.fcgi?artid=2684797\%5C\&tool=pmcentrez $\% 5$ C\&rendertype $=$ abstract \%20http : / / ww . sciencedirect . com/science / article / pii / S0197458007003508 (cit. on pp. 1, 3).

[7] Lonneke L. Verhoeff et al. "Effects of biofeedback on trunk sway during dual tasking in the healthy young and elderly". In: Gait $\mathcal{E}$ Posture 30.1 (July 2009), pp. 76-81. ISSN: 1879-2219. DOI: $10.1016 / \mathrm{j}$. gaitpost. 2009.03.002. URL: http : / / www . ncbi . nlm . nih . gov / pubmed / 19356934\%20http : / / ww . sciencedirect . com/science/article/pii / 0966636209000733 (cit. on p. 1).

[8] Steven W. Wu et al. "Torso-based tactile feedback system for patients with balance disorders". In: IEEE Haptics Symposium. 2010, pp. 359-362. ISBN: 9781424468225 (cit. on pp. 1, 3, 15). 
[9] Conrad Wall III, Diane M. Wrisley, and Kennyn D. Statler. "Vibrotactile tilt feedback improves dynamic gait index: a fall risk indicator in older adults". In: Gait \& Posture 30.1 (July 2009), pp. 16-21. ISSN: 1879-2219. DOI: 10.1016/ j . gaitpost . 2009 .02 .019. URL: http: / / www . pubmedcentral . nih . gov/ articlerender.fcgi?artid $=2752821 \% 5 \mathrm{C} \&$ tool=pmcentrez $\% 5 \mathrm{C} \&$ rendertype $=$ abstract \% 20http : / / www . sciencedirect . com / science / article / pii / S0966636209000617 (cit. on pp. 1, 16).

[10] Stacy J. Morris. "A shoe-integrated sensor system for wireless gait analysis and real-time therapeutic feedback". PhD thesis. Harvard-MIT, 2004. URL: http: //www . media.mit. edu/resenv/pubs/theses/sjmorrisSCDthesis . pdf (cit. on pp. 2, 4, 17).

[11] Paul Bach-y-Rita and Stephen W. Kercel. "Sensory substitution and the human-machine interface". In: Trends in Cognitive Sciences 7.12 (Dec. 2003), pp. 541-546. ISSN: 13646613. DOI: $10.1016 / \mathrm{j}$. tics . 2003 . 10 .013. URL: http://linkinghub.elsevier.com/retrieve/pii/S1364661303002900 (cit. on p. 4).

[12] H. Nakamura, T. Tsuchida, and Y. Mano. "The assessment of posture control in the elderly using the displacement of the center of pressure after forward platform translation." In: Journal of Electromyography and Kinesiology 11.6 (Dec. 2001), pp. 395-403. ISSN: 1050-6411. URL: http://www.ncbi.nlm.nih. gov/pubmed/11738952 (cit. on p. 5).

[13] Michailas Romanovas et al. "A study on indoor pedestrian localization algorithms with foot-mounted sensors". In: 2012 International Conference on Indoor Positioning and Indoor Navigation (IPIN) (Nov. 2012), pp. 1-10. DOI: 10.1109/IPIN . 2012.6418886. URL: http: // ieeexplore. ieee.org/lpdocs/ epic03/wrapper.htm?arnumber=6418886 (cit. on pp. 6, 18).

[14] John-Olof Nilsson et al. "Foot-mounted INS for everybody - an open-source embedded implementation". In: Proceedings of the 2012 IEEE/ION Position, Location and Navigation Symposium. Ieee, Apr. 2012, pp. 140-145. ISBN: 978-14673-0387-3. DOI: 10.1109/PLANS . 2012.6236875. URL: http://ieeexplore. ieee .org/lpdocs / epic03/wrapper.htm? arnumber=6236875 (cit. on pp. 6, 18).

[15] Eric Foxlin. "Pedestrian Tracking with Shoe-Mounted Inertial Sensors". In: IEEE Computer Graphics and Applications 25.December (2005), pp. 38-46 (cit. on pp. 6, 18).

[16] Y. Yang. "Spacecraft attitude determination and control: Quaternion based method". In: Annual Reviews in Control 36.2 (Dec. 2012), pp. 198-219. ISSN: 13675788. DOI: 10.1016/j. arcontrol.2012.09.003. URL: http://linkinghub . elsevier.com/retrieve/pii/S1367578812000387 (cit. on p. 6).

[17] Jack B. Kuipers. Quaternions and Rotation Sequences: A Primer with Applications to Orbits, Aerospace and Virtual Reality. Princeton University Press, 2002 (cit. on pp. 8, 11). 
[18] Mitchell Tyler, Yuri Danilov, and Paul Bach-y-Rita. "Closing an open-loop control system: vestibular substitution through the tongue". In: Journal of Integrative Neuroscience 2.02 (2003), pp. 159-164 (cit. on p. 15).

[19] Kathleen H. Sienko et al. "Effects of multi-directional vibrotactile feedback on vestibular-deficient postural performance during continuous multi-directional support surface perturbations". In: Journal of Vestibular Research : Equilibrium \&3 Orientation 18.5-6 (Jan. 2008), pp. 273-85. ISSN: 0957-4271. URL: http: //www.ncbi.nlm.nih.gov/pubmed/19542601 (cit. on p. 16).

[20] Beom-Chan Lee, Shu Chen, and Kathleen H. Sienko. "A wearable device for real-time motion error detection and vibrotactile instructional cuing". In: IEEE Transactions on Neural Systems and Rehabilitation Engineering 19.4 (Aug. 2011), pp. 374-81. ISSN: 1558-0210. DOI: 10.1109/TNSRE. 2011.2140331. URL: http://www.ncbi.nlm.nih.gov/pubmed/21511568 (cit. on p. 16).

[21] Conrad Wall III et al. "Balance prosthesis based on micromechanical sensors using vibrotactile feedback of tilt". In: IEEE Transactions on Biomedical Engineering 48.10 (Oct. 2001), pp. 1153-61. ISSN: 0018-9294. DOI: 10.1109/10. 951518. URL: http://www. ncbi.nlm.nih.gov/pubmed/11585039 (cit. on p. 16).

[22] Kathleen H. Sienko, M. David Balkwill, and Conrad Wall III. "Biofeedback improves postural control recovery from multi-axis discrete perturbations". In: Journal of Neuroengineering and Rehabilitation 9 (Jan. 2012), p. 53. ISSN: 17430003. DOI: 10 .1186/1743-0003-9-53. URL: http: //www . pubmedcentral . nih. gov / articlerender . fcgi ? artid $=3477042 \% 5 \mathrm{C} \&$ tool $=$ pmcentrez $\% 5 \mathrm{C} \&$ rendertype=abstract (cit. on p. 16).

[23] Stephanie Haggerty et al. "Effects of biofeedback on secondary-task response time and postural stability in older adults". In: Gait \& Posture 35.4 (Apr. 2012), pp. 523-8. ISSN: 1879-2219. DOI: 10.1016/j.gaitpost.2011.10.359. URL: http://www.ncbi.nlm.nih.gov/pubmed/22406291 (cit. on p. 16).

[24] Paul Fitzpatrick and Charles C. Kemp. "Shoes as a platform for vision". In: Proceedings of the Seventh IEEE International Symposium on Wearable Computers. Ieee, 2003, pp. 231-234. ISBN: 0-7695-2034-0. DOI: 10.1109/ISWC. 2003. 1241416. URL: http://ieeexplore.ieee.org/lpdocs/epic03/wrapper.htm? arnumber $=1241416$ (cit. on p. 17).

[25] Tri-Nhut Do and Young-Soo Suh. "Foot motion tracking using vision". In: 2011 IEEE 54th International Midwest Symposium on Circuits and Systems (MWS$C A S$ ). Vol. 00. IEEE, 2011, pp. 1-4. ISBN: 9781612848570. DOI: $10.1109 /$ MWSCAS. 2011.6026603 (cit. on p. 17).

[26] Tri Nhut Do and Young Soo Suh. "Gait analysis using floor markers and inertial sensors". In: MDPI Sensors 12.2 (Jan. 2012), pp. 1594-611. ISSN: 1424-8220. DOI: $10.3390 /$ s120201594. URL: http: / / www . pubmedcentral . nih . gov/ articlerender.fcgi ?artid $=3304129 \% 5 \mathrm{C} \&$ tool=pmcentrez $\% 5 \mathrm{C \& rendertype}=$ abstract (cit. on p. 17). 
[27] S. Ryan Edgar et al. "Wearable shoe-based device for rehabilitation of stroke patients". In: 32nd Annual International Conference of the IEEE EMBS. 2010, pp. 3772-3775. ISBN: 9781424441242. URL: http: / / ieeexplore. ieee.org/ xpls/abs\%5C_all.jsp?arnumber=5627577 (cit. on p. 17).

[28] Stacy J. Morris Bamberg et al. "Development of a quantitative in-shoe measurement system for assessing balance: sixteen-sensor insoles." In: Proceedings of the 28th IEEE EMBS Annual International Conference. Vol. 1. Jan. 2006, pp. 6041-4. ISBN: 1424400333. DOI: 10.1109/IEMBS . 2006.260424. URL: http: //www.ncbi.nlm.nih.gov/pubmed/17947179 (cit. on p. 18).

[29] Stacy J. Morris Bamberg et al. "Gait analysis using a shoe-integrated wireless sensor system". In: IEEE Transactions on Information in Biomedicine 12.4 (2008), pp. 413-423. URL: http://ieeexplore.ieee.org/xpls/abs\%5C_all. jsp?arnumber=4358886 (cit. on p. 18).

[30] Stacy J. Morris Bamberg et al. "The lower extremity ambulation feedback system for analysis of gait asymmetries: preliminary design and validation results". In: JPO Journal of Prosthetics and Orthotics 22.1 (Jan. 2010), pp. 31-36. ISSN: 1040-8800. DOI: 10 . 1097 / JPO . Ob013e3181 ccc065. URL: http: / / content . whealth . com / linkback / openurl ? sid = WKPTLP : landingpage $\% 5 \mathrm{C} \&$ an $=$ 00008526-201001000-00006 (cit. on p. 18).

[31] Adam M. Howell et al. "Kinetic gait analysis using a low-cost insole". In: IEEE Transactions on Biomedical Engineering 60.12 (Mar. 2013), pp. 3284-3290. ISSN: 1558-2531. DOI: 10 . 1109 / TBME . 2013 . 2250972. URL: http : / / www . ncbi.nlm.nih.gov/pubmed/23475336 (cit. on p. 18).

[32] Christian B. Redd and Stacy J. Morris Bamberg. "A wireless sensory feedback system for real-time gait modification". In: 33rd Annual International Conference of the IEEE EMBS. Vol. 2011. University of Utah, Salt Lake City, UT 84112, USA. Ieee, 2011, pp. 1507-1510. ISBN: 9781424441228. URL: http: // www . ncbi .nlm. nih. gov/pubmed/22254606\%20http : / / ieeexplore. ieee. org/xpls/abs\%5C_all.jsp?arnumber=6090344 (cit. on p. 18).

[33] Lucas Samuel Lincoln and Stacy J. Morris Bamberg. "Insole sensor system for real-time detection of biped slip". In: 32nd Annual International Conference of the IEEE EMBS. Vol. 2010. Jan. 2010, pp. 1449-52. ISBN: 9781424441242. DOI: 10.1109/IEMBS . 2010.5626859. URL: http://www.ncbi.nlm.nih.gov/ pubmed/21096354 (cit. on p. 18).

[34] Michael Angermann and Patrick Robertson. "FootSLAM: Pedestrian Simultaneous Localization and Mapping Without Exteroceptive Sensors - Hitchhiking on Human Perception and Cognition". In: Proceedings of the IEEE 100 (2012), pp. 1840-1848. DOI: 10.1109/JPROC.2012.2189785 (cit. on p. 18).

[35] Qilong Yuan and I.-Ming Chen. "3-D localization of human based on an inertial capture system". In: IEEE Transactions on Robotics 29.3 (June 2013), pp. 806-812. ISSN: 1552-3098. DOI: 10.1109/TRO.2013.2248535. URL: http: 
//ieeexplore. ieee.org/lpdocs/epic03/wrapper.htm?arnumber=6477156 (cit. on p. 18).

[36] Mitja Placer and Stanislav Kovačič. "Enhancing indoor inertial pedestrian navigation using a shoe-worn marker". In: MDPI Sensors 13.8 (Jan. 2013), pp. 983659. ISSN: 1424-8220. DOI: 10.3390/s130809836. URL: http://www . pubmedcentral. nih . gov/articlerender . fcgi ? artid $=3812582 \% 5 \mathrm{C} \&$ tool $=$ pmcentrez $\%$ C \& rendertype=abstract (cit. on p. 19).

[37] Patricia Giacoman Zarzar. "Force sensing insole for a balance enhancement system". MA thesis. Ottawa: Carleton University, Sept. 2014 (cit. on p. 20).

[38] What is a Raspberry Pi? July 2014. URL: http: / / www . raspberrypi .org/ help/what-is-a-raspberry-pi/ (cit. on p. 29).

[39] Raspberry Pi Hardware. July 2014. URL: http : / / www . raspberrypi . org / documentation/hardware/raspberrypi/README.md (cit. on p. 30).

[40] Camera Module Setup. July 2014. URL: http://www.raspberrypi.org/help/ camera-module-setup/ (cit. on p. 31).

[41] Color CMOS QSXGA (5-megapixel) Image Sensor with OmniBSI Technology. July 2014. URL: http://www. ovt.com/products/sensor .php?id=66 (cit. on p. 32).

[42] Photojojo iPhone and Android Lens Series. July 2014. URL: http://photojojo. com/store/awesomeness/cell-phone-lenses/ (cit. on p. 31).

[43] Linux and the GNU System. July 2014. URL: https: //www . gnu .org/gnu/ linux-and-gnu.html (cit. on p. 40).

[44] Juho Kannala and Sami S. Brandt. "A generic camera model and calibration method for conventional, wide-angle, and fish-eye lenses". In: IEEE Transactions on Pattern Analysis and Machine Intelligence 28.8 (2006), pp. 1335-1340 (cit. on pp. 56, 59, 61).

[45] Jonathan Fabrizio and Jean Devars. "An analytical solution to the perspectiveN-point problem for common planar camera and for catadioptric sensor". In: International Journal of Image and Graphics 08.01 (Jan. 2008), pp. 135-155. ISSN: 0219-4678. DOI: 10 . 1142 / S0219467808003015. URL: http : / / wWw . worldscientific.com/doi/abs/10.1142/S0219467808003015 (cit. on pp. 64, $66,89)$.

[46] F. Landis Markley and Daniele Mortari. "Quaternion attitude estimation using vector observations". In: Journal of the Astronautical Sciences 48.2 (2000), pp. 359-380 (cit. on p. 68). 


\section{A. Software}

For convenience, the source code for the shoevision and integrator programs (described in Chapters 2 and 3) have been embedded within the electronic version of this document. Please right click the appropriate links to save the compressed archives. 


\section{B. Calibration Results}

Table B.1.: Location of the eight markers in the camera frame of each shoe $\left(\mathbf{v}_{C n, C}\right)$. All units are in millimeters.

\begin{tabular}{cccccrc}
\hline Marker & \multicolumn{3}{c}{ Left Shoe } & \multicolumn{3}{c}{ Right Shoe } \\
\hline \hline $\mathrm{n}$ & $\mathrm{x}$ & $\mathrm{y}$ & \multicolumn{1}{c}{$\mathrm{z}$} & \multicolumn{1}{c}{$\mathrm{x}$} & $\mathrm{y}$ & $\mathrm{z}$ \\
\hline 1 & 23.98 & 14.15 & -12.43 & -24.36 & 9.06 & -11.74 \\
\hline 2 & 47.51 & -25.89 & -9.73 & -47.11 & -31.10 & -11.00 \\
\hline 3 & 46.34 & 52.97 & -11.59 & -46.74 & 48.97 & -13.660 \\
\hline 4 & -22.16 & 12.43 & -11.62 & 21.77 & 9.80 & -10.18 \\
\hline 5 & -126.65 & 114.54 & -32.77 & 122.49 & 113.37 & -48.84 \\
\hline 6 & -141.73 & 145.97 & -88.68 & 134.80 & 139.84 & -110.03 \\
\hline 7 & -154.42 & 69.52 & -49.42 & 151.25 & 68.07 & -61.37 \\
\hline 8 & -170.11 & 100.94 & -105.72 & 165.48 & 94.11 & -121.55 \\
\hline
\end{tabular}


Table B.2.: Parameters used in Fabrizio's solution for the front and rear marker set of each shoe.

\begin{tabular}{ccccc}
\hline Parameter & \multicolumn{2}{c}{ Left Shoe } & \multicolumn{2}{c}{ Right Shoe } \\
\hline \hline & Front & Rear & Front & Rear \\
\hline$L_{12}(\mathrm{~mm})$ & 46.529499 & 65.882671 & 46.169417 & 67.799119 \\
\hline$K_{1}$ & -0.920529 & -0.999328 & -1.011080 & -0.969208 \\
\hline$K_{2}$ & -0.952196 & 0.992119 & -0.983222 & 0.979328 \\
\hline
\end{tabular}

Table B.3.: Coordinate system transformation from the camera frame to the rear frame for each shoe.

\begin{tabular}{|c|c|c|c|c|c|c|c|c|}
\hline & \multirow[b]{2}{*}{$\mathbf{v}_{O C}$} & & \multicolumn{3}{|c|}{ Left Shoe } & \multicolumn{3}{|c|}{ Right Shoe } \\
\hline \multirow{3}{*}{$\Lambda_{O C}$} & & & $(-49.57$ & 40.08 & $-229.32)$ & $(-125.30$ & 34.09 & $-198.34)$ \\
\hline & \multirow{2}{*}{$\mathbf{q}_{O C}$} & $\mathbf{q}_{O C, s}$ & \multicolumn{3}{|c|}{-0.1269} & \multicolumn{3}{|c|}{-0.3896} \\
\hline & & $\mathbf{q}_{O C, v}$ & $(0.5203$ & -0.6575 & $-0.5299)$ & $(-0.8639$ & 0.3183 & $0.0257)$ \\
\hline
\end{tabular}


Table B.4.: Camera model parameters for both shoes.

\begin{tabular}{|c|c|c|}
\hline Parameter & Left Shoe & Right Shoe \\
\hline$u_{0}$ & $1.298383 \mathrm{e}+03$ & $1.296907 \mathrm{e}+03$ \\
\hline$v_{0}$ & $9.326712 \mathrm{e}+02$ & $9.082945 \mathrm{e}+02$ \\
\hline$S_{1}$ & $1.229786 \mathrm{e}-03$ & $7.802570 \mathrm{e}-04$ \\
\hline$S_{2}$ & $1.224633 \mathrm{e}-03$ & $7.746590 \mathrm{e}-04$ \\
\hline$L_{1}$ & $1.312178 \mathrm{e}-02$ & $-2.026610 \mathrm{e}-02$ \\
\hline$L_{2}$ & $-5.966697 \mathrm{e}-02$ & $2.293147 \mathrm{e}-01$ \\
\hline$L_{3}$ & $1.509694 \mathrm{e}-02$ & $-9.741788 \mathrm{e}-02$ \\
\hline$I_{1}$ & $\begin{array}{l}-1.637937 \mathrm{e}-02 \\
\end{array}$ & $5.542409 \mathrm{e}-02$ \\
\hline$I_{2}$ & $-9.718507 \mathrm{e}-02$ & $1.317064 \mathrm{e}-01$ \\
\hline$I_{3}$ & $9.608476 \mathrm{e}-02$ & $-7.301364 \mathrm{e}-02$ \\
\hline$I_{4}$ & $-7.052286 \mathrm{e}-02$ & $6.599093 \mathrm{e}-03$ \\
\hline$M_{1}$ & $-3.211818 \mathrm{e}-02$ & $-3.412568 \mathrm{e}-02$ \\
\hline$M_{2}$ & $-8.021284 \mathrm{e}-02$ & $-3.102555 \mathrm{e}-02$ \\
\hline$M_{3}$ & $4.128072 \mathrm{e}-03$ & $-5.239253 \mathrm{e}-03$ \\
\hline$J_{1}$ & $-5.004716 \mathrm{e}-02$ & $-1.879984 \mathrm{e}-02$ \\
\hline$J_{2}$ & $6.504818 \mathrm{e}-02$ & $1.230594 \mathrm{e}-01$ \\
\hline$J_{3}$ & $-4.216715 \mathrm{e}-04$ & $-2.720506 \mathrm{e}-02$ \\
\hline$J_{4}$ & $-2.048358 \mathrm{e}-02$ & $-7.492199 \mathrm{e}-03$ \\
\hline$D_{1}$ & $9.950372 \mathrm{e}-01$ & $1.589756 \mathrm{e}+00$ \\
\hline$D_{2}$ & $-1.862519 \mathrm{e}-02$ & $-1.947739 \mathrm{e}-01$ \\
\hline$D_{3}$ & $-2.308576 \mathrm{e}-03$ & $3.509328 \mathrm{e}-01$ \\
\hline$D_{4}$ & $5.880145 \mathrm{e}-03$ & $-3.680262 \mathrm{e}-01$ \\
\hline$D_{5}$ & $1.742696 \mathrm{e}-04$ & $2.514129 \mathrm{e}-01$ \\
\hline
\end{tabular}




\section{Additional Test Results}

The following figures show the estimated path of 5 subjects calculated for the long walk experiment described in Section 5.3.

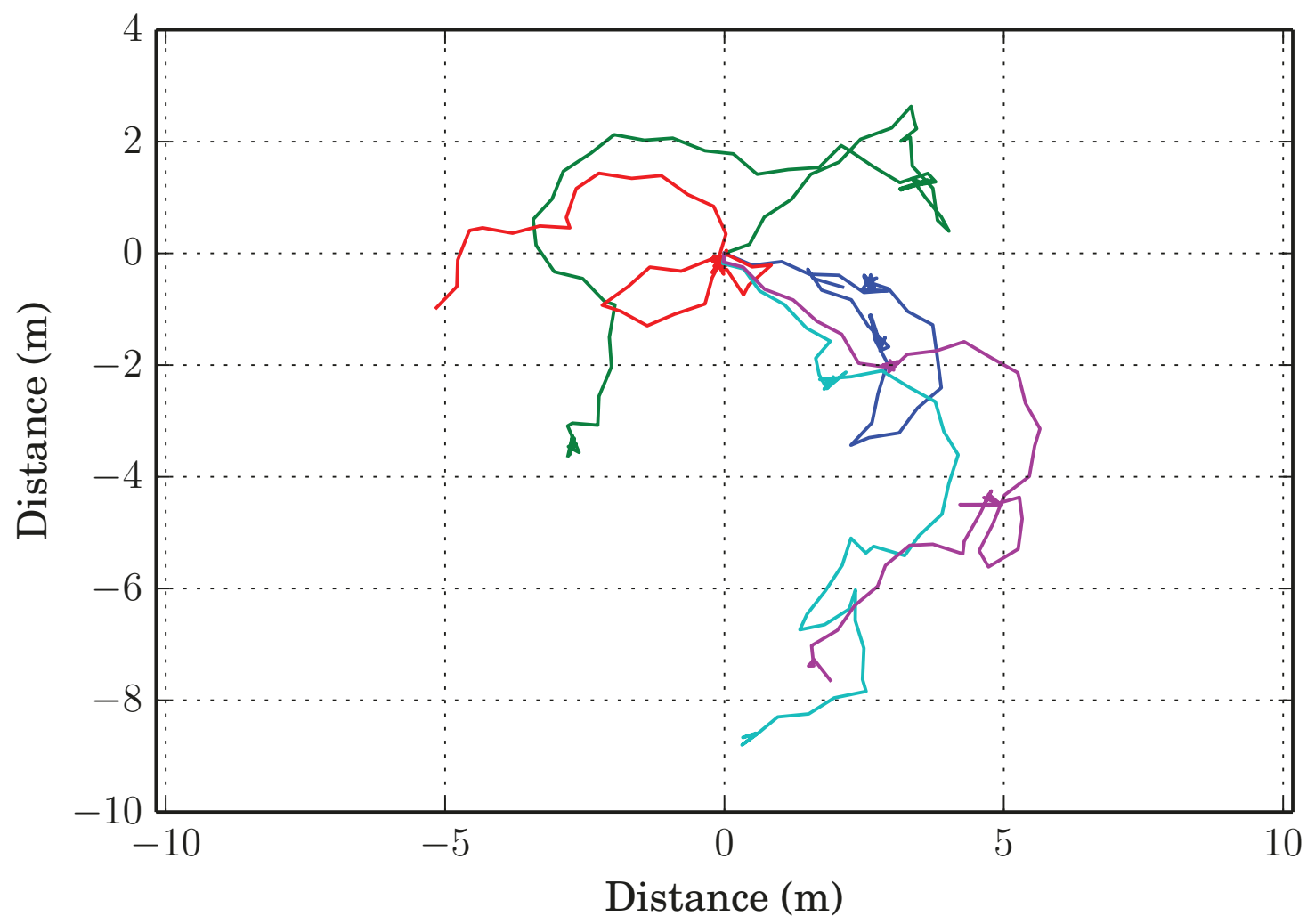

Figure C.1.: The path of each circuit made by subject 1 estimated from the tracking algorithm. Starting point for each circuit is at $(0,0)$. Each colour represents a different circuit. 


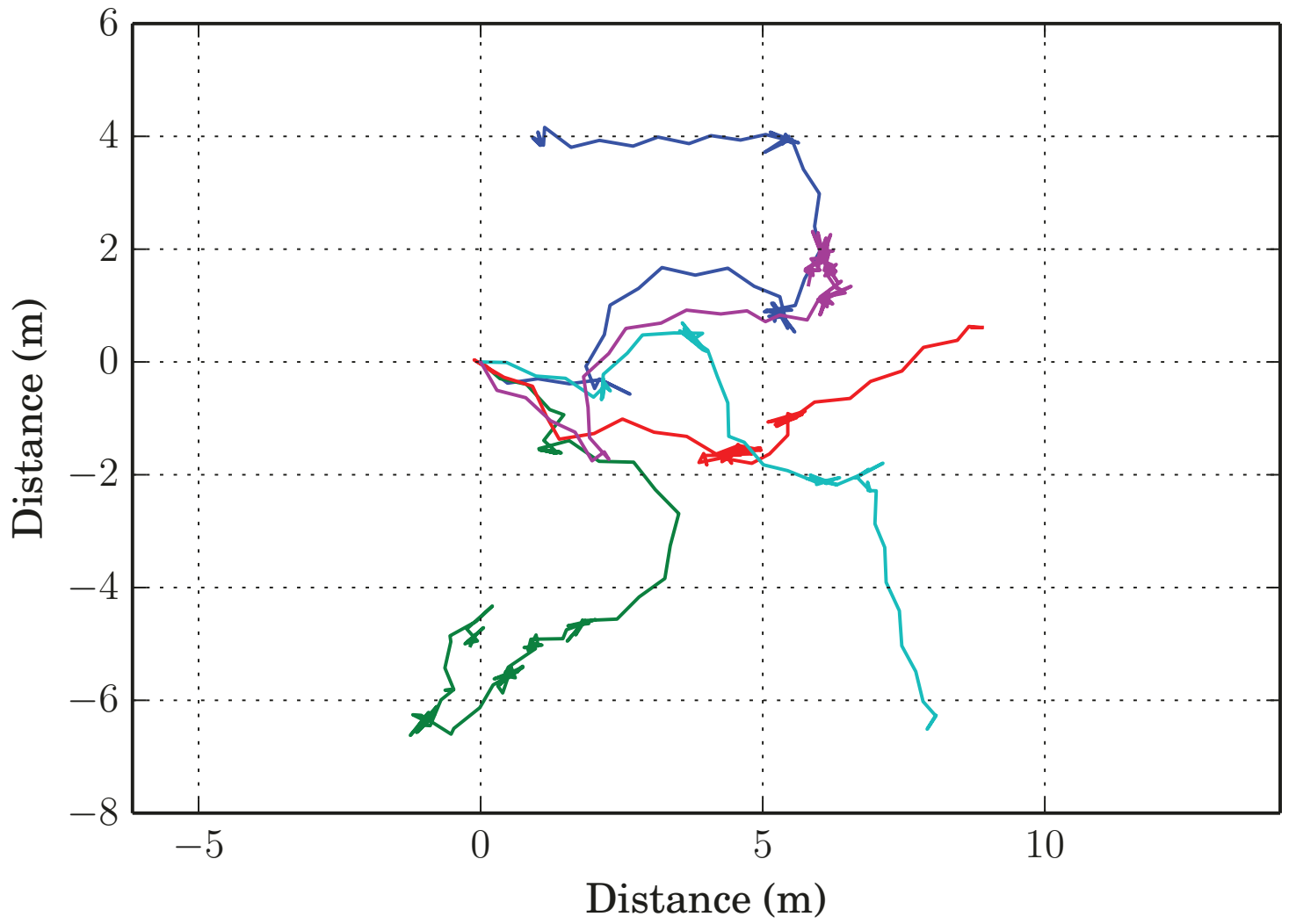

Figure C.2.: The path of each circuit made by subject 2 estimated from the tracking algorithm. Starting point for each circuit is at $(0,0)$. Each colour represents a different circuit. 


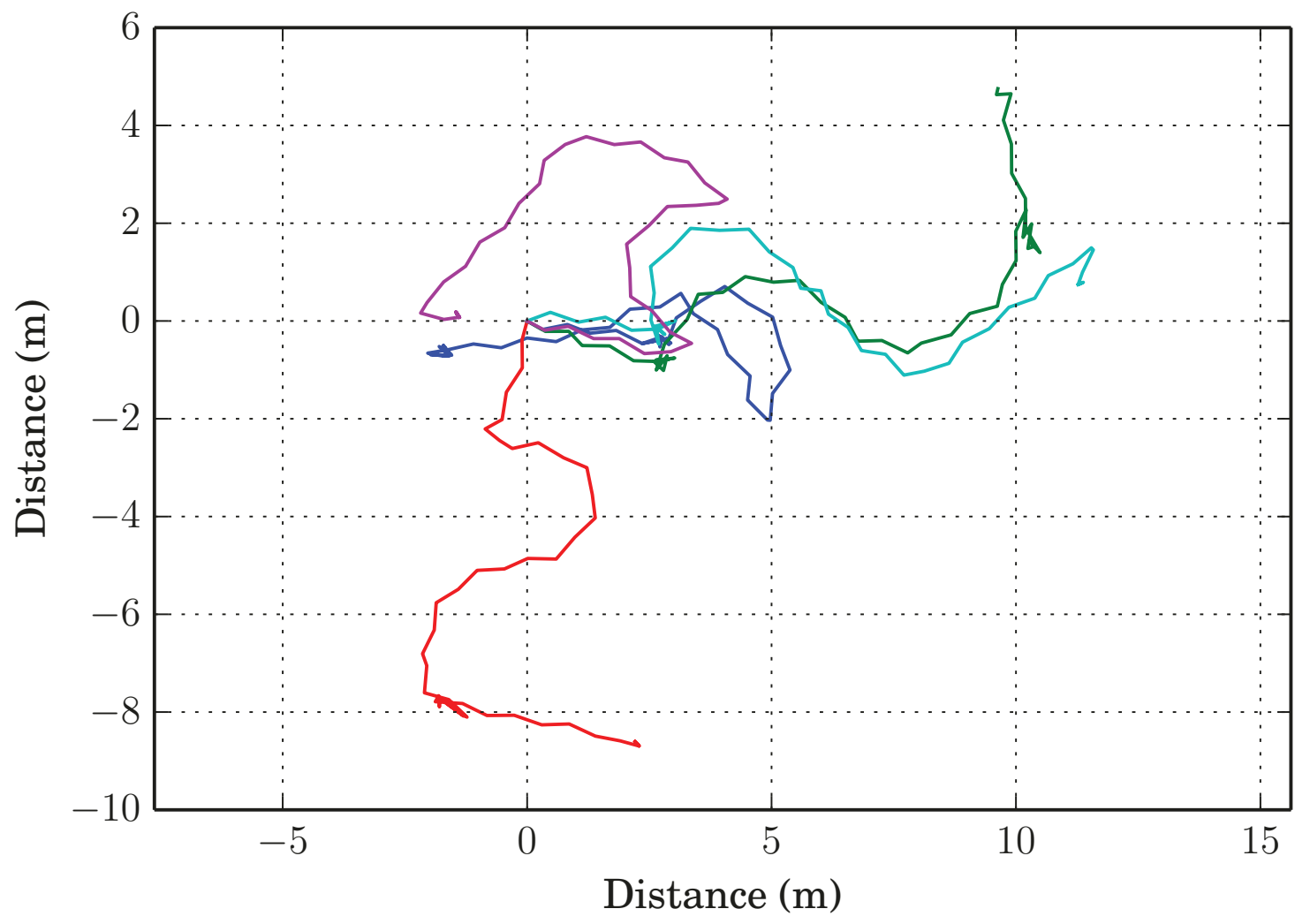

Figure C.3.: The path of each circuit made by subject 3 estimated from the tracking algorithm. Starting point for each circuit is at $(0,0)$. Each colour represents a different circuit. 


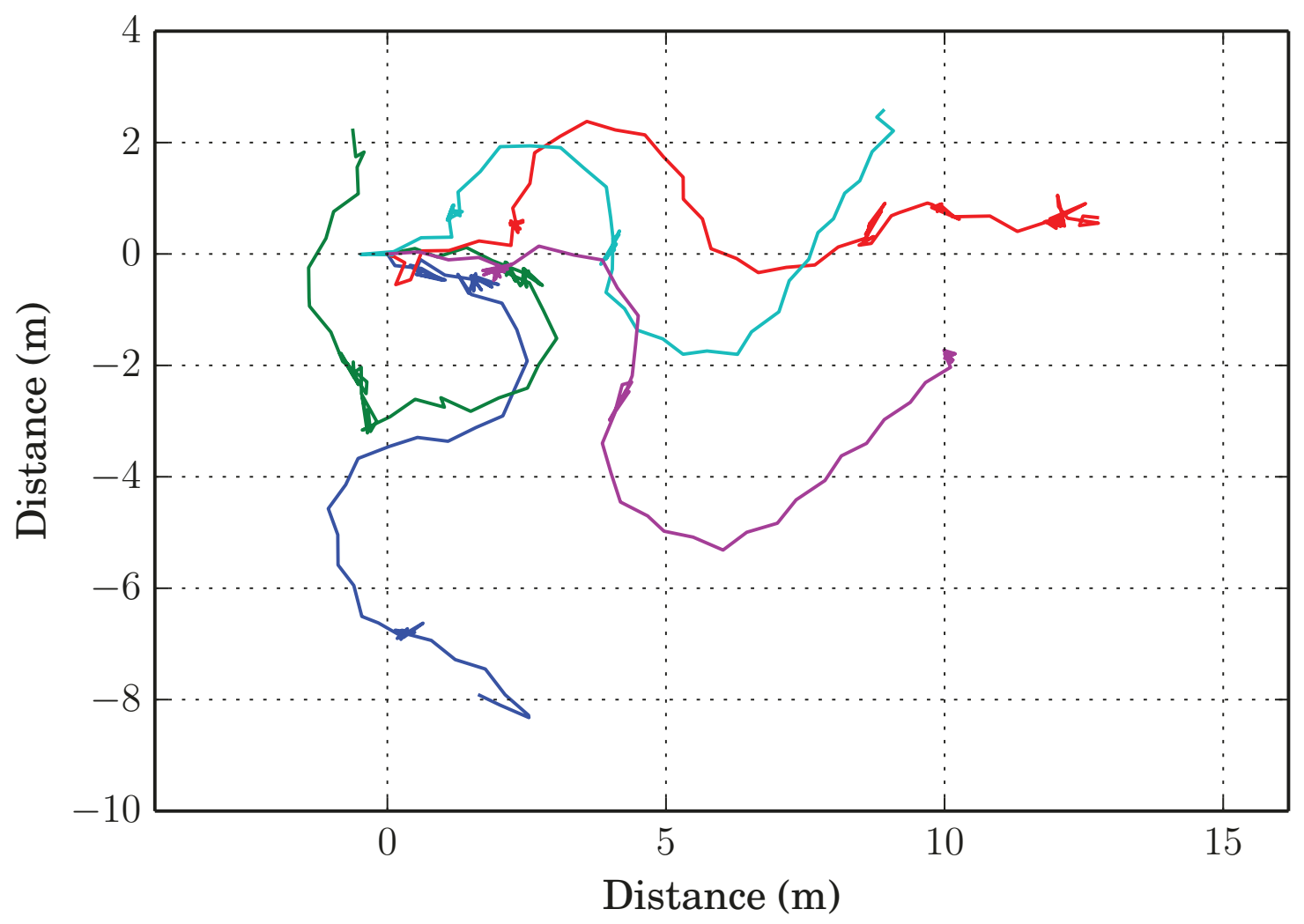

Figure C.4.: The path of each circuit made by subject 4 estimated from the tracking algorithm. Starting point for each circuit is at $(0,0)$. Each colour represents a different circuit. 


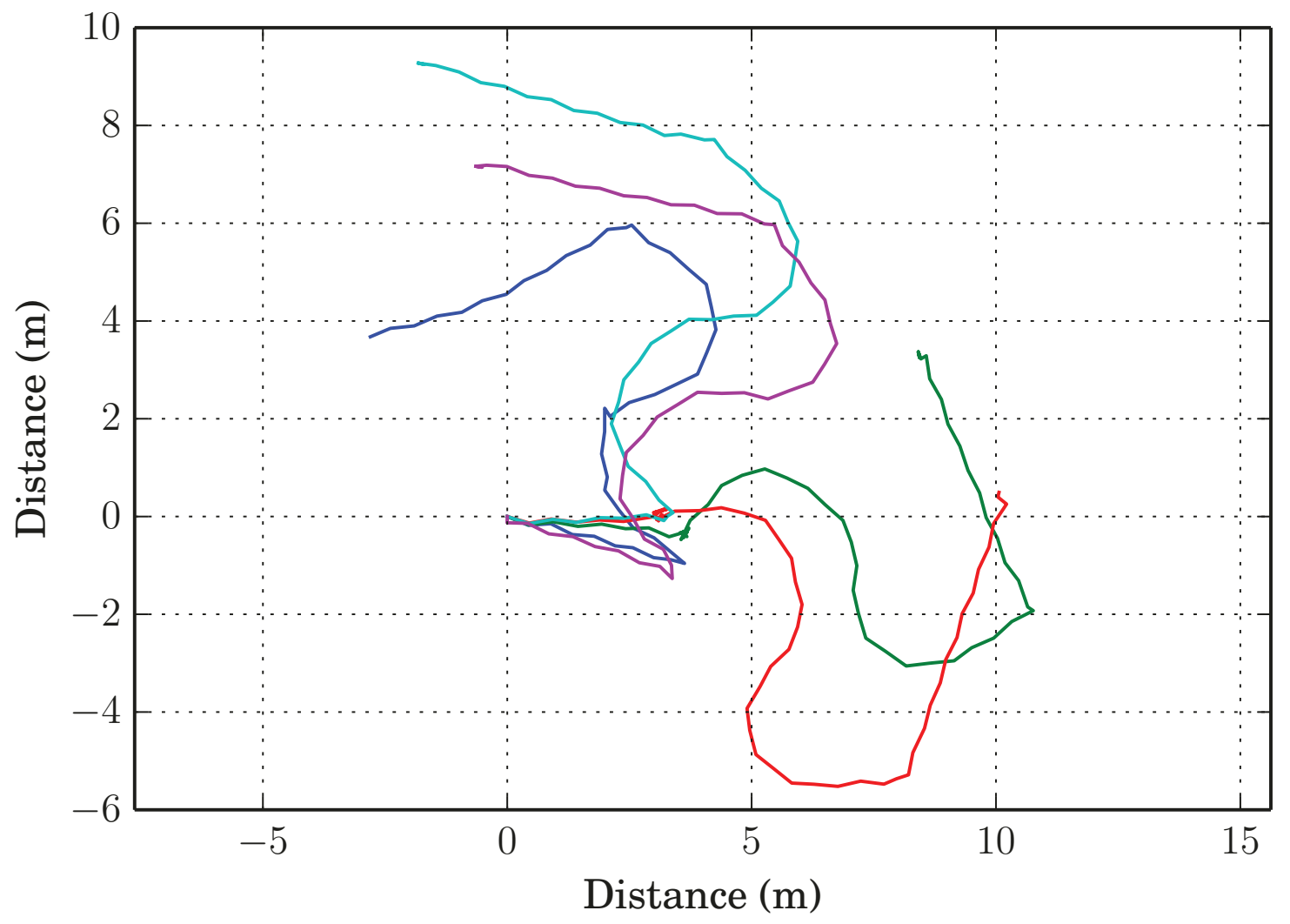

Figure C.5.: The path of each circuit made by subject 5 estimated from the tracking algorithm. Starting point for each circuit is at $(0,0)$. Each colour represents a different circuit. 